\title{
STRUCTURE, DYNAMICS AND STATISTICAL PROPERTIES OF GALAXIES
}

\author{
G. DE VAUCOULEURS
}

The University of Texas at Austin, Tex., U.S.A.

\begin{abstract}
The following empirical or physical parameters describing individual and statistical properties of galaxies are reviewed: morphological type including new or revised types; intrinsic luminosity distribution functions of two main components (spheroid and disk); true ellipticities of different types; characteristic scale parameters (effective diameter and luminosity density) of spheroidal and flat systems; masses and densities of spheroidal, disk and mixed systems from velocity dispersion and rotation velocities; rotation periods, maximum rotation velocities, angular velocities and momenta of different types; neutral hydrogen masses and densities; spectral energy distributions and colour indices; number of independent parameters from principal component (factor) analysis; luminosity functions and selection effects; clustering and space distribution; maximum density-radius and velocity dispersion-radius relations in systems of galaxies.
\end{abstract}

\section{Introduction}

Galaxies may be regarded, in one view, merely as a bewildering collection of different individual objects each with its own peculiarities, but in another view, adopted here, as forming a class of physical systems characterizable by a small set of empirical or basic physical parameters (Table I). The average values and distribution functions of these quantities once known may be used as inputs to theories of galactic structure and evolution and, at least ideally, they should be predictable as outputs of realistic cosmological models.

In particular we need to define

(1) a characteristic length or scale factor $L^{*}$, for example the effective radius $r_{\mathrm{e}}^{*}$ of the volume $V_{\mathrm{e}}$ within which half the total mass is located or within which half the

TABLE I

Dominant empirical and physical parameters

\begin{tabular}{|c|c|c|c|}
\hline Parameters & & Stars & Galaxies \\
\hline Empirical & $\begin{array}{l}\text { Present } \\
\text { Initial }\end{array}$ & $\begin{array}{l}\left\{\begin{array}{l}\text { Spectral type } \\
\text { Luminosity } \\
\text { Mass } \\
\text { Radius } \\
\text { Temperature } \\
\text { Pressure } \\
\text { Angular Momentum }\end{array}\right. \\
\left\{\begin{array}{l}\text { Chemical composition } \\
\text { Mass function } \\
\text { (Model of Protogalaxy) }\end{array}\right.\end{array}$ & 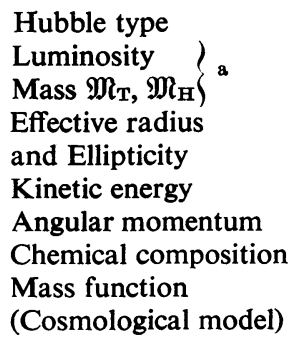 \\
\hline
\end{tabular}

a and radial density distribution functions. 
total power or bolometric luminosity is emitted. When this information is not yet accessible, we may use as substitutes some parameters of the optical luminosity distribution, for example the $e$-folding length $\Lambda$ or scale height in an exponential distribution (Freeman, 1970), the structural length in a quasi-isothermal distribution (Zwicky, $1937,1957)$ or some other metric scale factor ${ }^{\ddagger}$

(2) A characteristic mass $\mathfrak{M}^{*}$, perhaps the total mass $\mathfrak{M}_{\mathrm{T}}$, if it can be defined and evaluated, or at least some indicative mass value $\mathfrak{M}_{i}$ derived by a fixed rule from length and velocity data, for example $\mathfrak{M}_{i}=V_{\mathrm{M}}^{2} R_{\mathrm{M}} / G \mu$, where $V_{\mathrm{M}}=V\left(R_{\mathrm{M}}\right)$ is the maximum rotational velocity in a flat rotating disk (Bottinelli et al., 1968), or $\mathfrak{M}_{i}=\lambda \sigma_{\mathrm{v}}^{2} r_{\mathrm{e}}^{2} / G$, where $\sigma_{\mathrm{v}}$ is the central velocity dispersion in a spheroidal system of effective radius $r_{\mathrm{e}}$ (Poveda, 1958; Poveda et al., 1960); $\lambda$ and $\mu$ are empirical factors chosen to achieve approximate equality between $\mathfrak{M}_{i}$ and $\mathfrak{M}_{\mathrm{T}}$, the latter derived from more realistic models.

If both $\mathfrak{M}_{\mathrm{T}}$ and the mass distribution function $\mathfrak{M}(r) / \mathfrak{M}_{\mathrm{T}}$ can be estimated, the ratio $\varrho_{\mathrm{e}}=\mathfrak{M}_{\mathrm{T}} / 2 V_{\mathrm{e}}$ is, at least in principle, a well-defined measure of the average (effective) density of the system.

(3) A characteristic time scale $T^{*}$, be it some typical rotation period, for example that corresponding to the maximum rotational velocity $V_{\mathrm{M}}\left(R_{\mathrm{M}}\right)$ in flat systems, or some typical crossing time, for example $r_{\mathrm{e}}^{*} / \sigma_{\mathrm{v}}$ in spheroidal systems.

If both $\varrho_{\mathrm{e}}$ and $\sigma_{\mathrm{v}}$ can be evaluated, two scale factors of great dynamical interest may be derived: the Jeans length $L_{\mathrm{e}}=\left(\sigma_{\mathrm{v}}^{2} / G \varrho_{\mathrm{e}}\right)^{1 / 2}$ and the free fall time $T_{\mathrm{e}}=\left(G \varrho_{\mathrm{e}}\right)^{-1 / 2}$.

(4) The total energy output $\varepsilon_{\mathrm{T}}$ in the form of electromagnetic radiation of all wavelengths (bolometric luminosity) and its normalized spectral distribution $\varepsilon(\lambda) / \varepsilon_{\mathrm{T}}$. When it cannot be evaluated, the total absolute magnitude $M$ in one of the standard colour bands ( $B$ or $V$ ) and at least two colour indices, say $B-V$ and $U-B$, are useful though rather unsatisfactory substitutes.

An important derived quantity is the (bolometric) mass-luminosity ratio $f^{*}=\mathfrak{M}_{\mathrm{T}} / \mathfrak{L}^{*}$ or at least its optical counterpart, say $f_{\mathrm{v}}=\mathfrak{M}_{\mathrm{T}} / \mathfrak{L}_{\mathrm{v}}$, corrected for self-absorption if appropriate (both in solar units).

(5) The normalized laws of luminosity and mass density distributions in galaxies of different morphological types, if possible per unit volume $J(\mathbf{R})$ and $\mu(\mathbf{R})$ - or at least per unit area in the face-on projection $J(r)$ and $\mu(r)$-from which the radial dependence of the mass-luminosity ratio $f(r)$ is derived.

(6) The normalized laws of density distribution of interstellar neutral hydrogen in galaxies of different types $H(\mathbf{R})$ and the total hydrogen mass $\mathfrak{M}_{\mathrm{H}}$. Important derived quantities are the ratio of hydrogen mass to total mass $h=\mathfrak{M}_{\mathrm{H}} / \mathfrak{M}_{\mathrm{T}}$ and the distanceindependent ratio of hydrogen to luminosity $g=\mathfrak{M}_{\mathrm{H}} / \mathfrak{L}^{*}$ (both in solar units).

Some other derived quantities that are more or less directly accessible are also important, for example the total net angular momentum $A$ of a galaxy (or the average

\# Isophotal diameters are, in general, less satisfactory size indicators since they depend on both luminosity density and space orientation as well as pure scale factors. Commonly quoted 'photographic' diameters are even less satisfactory since they depend additionally on the apparent luminosity gradient (de Vaucouleurs, 1959a; Heidmann et al., 1971). 
angular momentum per unit mass) may give clues to initial conditions since it should be conserved in the evolution of an isolated galaxy (although galaxies can hardly be regarded as 'isolated' on a time scale of $10^{10} \mathrm{yr}$ ).

Finally, a property of great physical and dynamical significance, but which does not easily yield to quantitative expression or measurement, is the structural or morphological type $T$, e.g. in the revised Hubble scheme (de Vaucouleurs 1956a, 1959a, 1962, 1964; Sandage, 1961, 1973). In particular the stage $t$ along the Hubble sequence, from ellipticals to Magellanic irregulars through lenticulars and spirals, correlates well with a number of measurable parameters, but cannot yet be precisely derived from such measurements. In the present state of the art, subjective classifications by experienced observers agree better than objective estimates from quantitative correlations (de Vaucouleurs, 1961; Corwin, 1968, 1970; Heidmann et al., 1971; Brosche, 1973).

The present review will deal mainly with the 'normal' galaxies which are still by far the most common type of extragalactic objects and, as far as we know, are the major contributors to the mean density of space.

\subsection{REMARKS ON SELECTION EFFECTS}

The discovery of galaxies is severely limited by observational selection of surface brightness and apparent diameters. Figure 1, adapted from Arp (1965), illustrates this point. Objects having an average surface luminosity less than $\mu_{B} \simeq 27 \mathrm{mag}\left(\operatorname{arc~sec}^{-2}\right)$ $\left(\simeq 1 \mathfrak{L}_{\odot} \mathrm{pc}^{-2}\right)$ are not optically detectable by present techniques. Objects having an apparent diameter less than $\sim 1^{\prime \prime}$ are not readily distinguishable from stars with current instruments. It is probably not by accident that the average surface brightness of the so-called 'normal' galaxies is only slightly above that of the night sky (de Vaucouleurs, 1957a).

Further selection effects arise in the formation of catalogues. No galaxy catalogue is complete to any given total magnitude or even $50 \%$ complete at some fixed magnitude. The effective limit is mainly a function of surface brightness $b$ for large objects, of total luminosity $b D^{2}$ for smaller objects, and of apparent diameter $D$ only for very small objects which necessarily must have high $b$. It is secondarily a function of shape and light concentration (i.e. morphological type) and subject to external factors such as star field density and interstellar extinction. There is very little quantitative information on the completeness factors involved and the relative space densities of different galaxy types other than 'normal' remain highly uncertain. All the distribution functions describing the statistical properties of galaxies are subject to this fundamental bias of unknown magnitude.

\section{Morphology and Classification}

The revised Hubble system is applicable to $95 \%$ or more of the galaxy population; its general validity and basic significance is demonstrated by the fact that the stage $T$ along the Hubble sequence from $\mathrm{E}$ to $\mathrm{I}$ (or on a convenient numerical scale from 


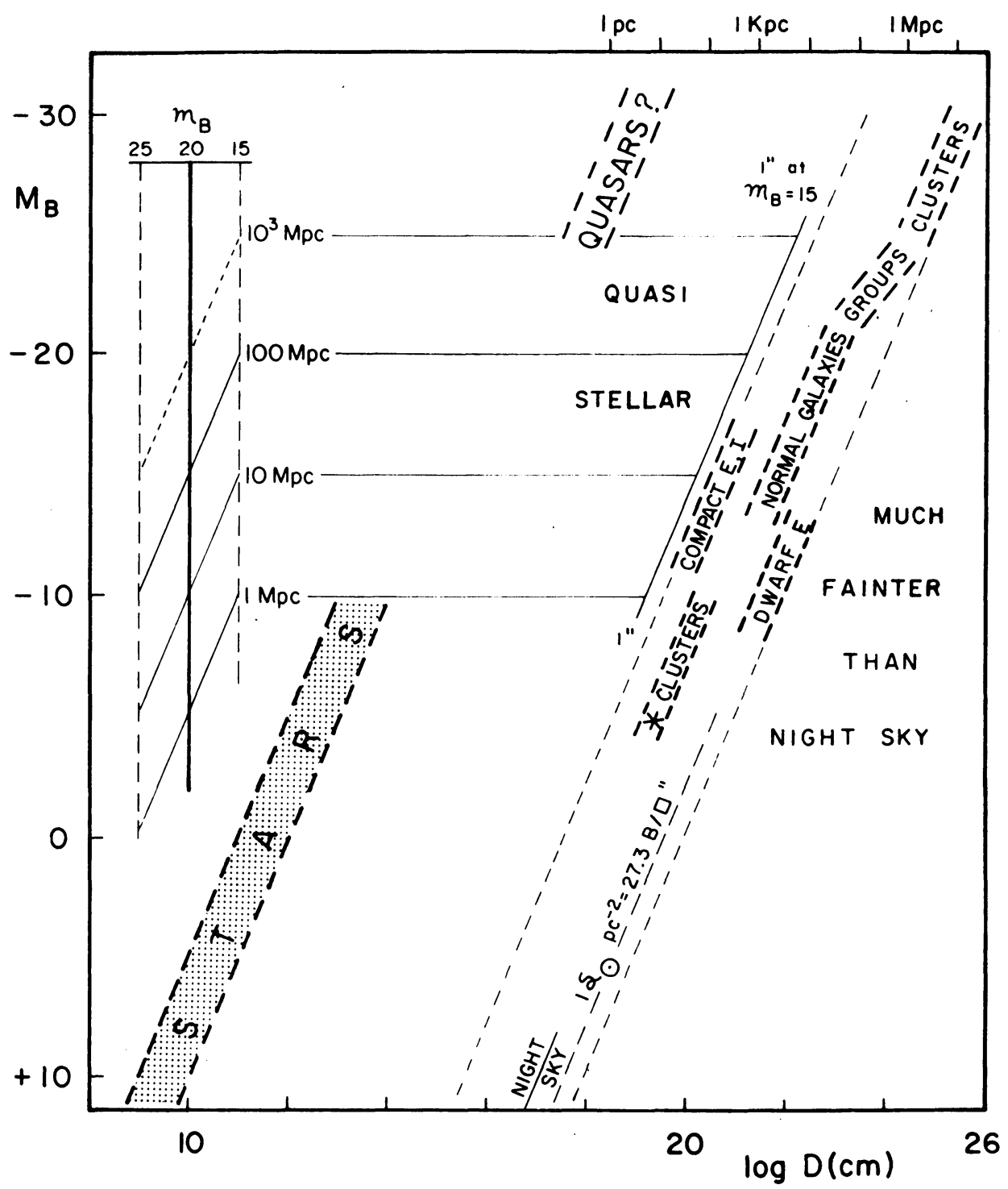

Fig. 1. Luminosity-diameter relation for stars and galaxies, adapted from Arp (1965). Normal galaxies and star clusters are restricted to a narrow range of surface brightness close to that of the night sky. Optical detection of galaxies smaller than $\sim 1^{\prime \prime}$ or fainter than $\sim 1 \mathfrak{I}_{\odot} \mathrm{pc}^{-2}$ is not possible by current techniques. Relation between apparent magnitude $m_{\mathrm{B}}$, absolute magnitude $M_{\mathrm{B}}$, linear diameter $D$ and distance in $\mathrm{Mpc}$ for a $\mathbf{1}^{\prime \prime}$ apparent diameter is illustrated. 
$t=-5$ to +10 ) correlates well with several fundamental parameters such as photometric structure, colour index, hydrogen content, etc. $¥$ On the other hand the structural differences between families $(A, A B, B)$ and varieties $(r, r s, s)$ of lenticulars and spirals, that is the presence or absence of a bar or a ring, seem to reflect relatively minor differences of detailed dynamics rather than basic differences in physical properties and composition. In brief, the long axis of the 3-dimensional classification volume relates to the basic physics of galaxies, while the cross-section displays dynamical details.

The quantitative studies of recent years suggest some minor corrections and additions to the sequence of stages. For example Morgan's $\mathrm{cD}$ galaxies fit best at stage $t=-4\left(\mathrm{E}^{+}\right)$; compact ellipticals may be added as an extension $\left(\mathrm{E}^{-}\right)$of the sequence to $t=-6$. Similarly, the dwarf compact Magellanic irregulars recently described as isolated extragalactic $\mathbf{H}$ II regions (Sargent and Searle, 1970) may be included in the revised Hubble sequence as an additional step $\left(\operatorname{Im}^{+}\right), t=11$, beyond stage $\operatorname{Im}(t=10)$. Low density irregulars of the IC 2574 and W-L-M nebula type are already recognized, being designated as $\mathrm{dIm}$.

Irregular galaxies of the rare, non-Magellanic type, originally described by Hubble (1926) as merely 'chaotic', may be designated as 'type II' (Holmberg, 1950) or preferably I0 (de Vaucouleurs, 1963) because, except for their dust lanes, their structure and physical properties resemble most closely those of normal galaxies near the transition stage So/a, $t=0$, between lenticulars and spirals (Heidmann et al., 1971).

The revised classification sequence appears in Table II. A number of recently reported 'new' galaxy types deserve some discussion:

(a) Gamma $(\gamma ; \gamma$-R) types: as described by Vorontsov-Velyaminov (1962) $\gamma$-types

TABLE II

Revised Hubble sequence

\begin{tabular}{|c|c|c|c|c|c|c|c|c|c|c|c|c|}
\hline Stage & $t$ & -6 & -5 & -4 & -3 & -2 & -1 & & & & & \\
\hline Type & $\mathrm{T}$ & $\begin{array}{l}\mathrm{E}^{-} \\
\mathrm{a}\end{array}$ & $\begin{array}{l}E \\
b\end{array}$ & $\begin{array}{l}\mathrm{E}^{+} \\
\mathbf{c}\end{array}$ & $\mathrm{L}^{-}$ & $\mathbf{L}^{0}$ & $\mathbf{L}^{+}$ & & & & & \\
\hline Stage & 0 & 1 & 2 & 3 & 4 & 5 & 6 & 7 & 8 & 9 & 10 & 11 \\
\hline Type & $\underset{\mathrm{d}}{\mathrm{S} 0 / \mathrm{a}}$ & $\mathrm{Sa}$ & $\mathrm{Sab}$ & $\mathrm{Sb}$ & Sbc & Sc & Scd & Sd & $\mathrm{Sdm}$ & $\mathrm{Sm}$ & $\mathrm{Im}_{\mathrm{e}}^{\mathrm{Im}}$ & $\operatorname{Im}^{+}$ \\
\hline
\end{tabular}

a compact $\mathrm{E}$.

$b$ and $\mathrm{dE}$.

c Morgan's cD.

d also I0.

e and dIm.

e compact Im.

$\ddagger$ The precision with which $t$ may be estimated by a trained observer on plates having adequate resolution ( $\sim 10^{4}$ picture elements in area) is indicated by the standard error $\sigma_{t}=0.75$ (or $5 \%$ of the 15-step range) objectively derived through factor analysis by Brosche (1973) (Section 11). This is consistent with earlier estimates $\left(\sigma_{t} \simeq 1\right)$ from intercomparison of independent classifications by several observers (de Vaucouleurs, 1962; Corwin, 1970). 
are mainly late-type barred spirals, especially of the Magellanic type SBm. The Large Cloud itself, photographed at low resolution and with a short exposure, is a good example (Figure 2). The arms $\boldsymbol{B}_{1}, \boldsymbol{B}_{2}$ form the branches of the $\gamma$, the bar $\boldsymbol{A}$ is the stem. When on longer exposures the circular outer loop $C$ is visible, the $\gamma$-R type is produced. In some cases $\gamma$-R may describe other galaxy types with a cardioid outer ring, e.g. NGC 3368 (Sandage, 1961). Many small Magellanic barred spirals appear $\gamma$-shaped on the Palomar Sky Survey prints.

(b) Ring galaxies. The ring-type galaxies of which Mayall's nebula was the first example (Smith, 1941; Baade and Minkowski, 1954; Arp, 1966), and the chaotic irregulars rich in emission regions, of which NGC 2444-45 and 6438 are two well studied examples (Sandage, 1963; Sersic, 1966, 1968a; Burbidge and Burbidge, 1972), appear to result from the interaction of a spiral or Magellanic-type system with a heavier and denser elliptical or lenticular galaxy (Sandage, 1963), or they may be produced by collisions between spirals and intergalactic hydrogen clouds $\ddagger$ (Freeman and de Vaucouleurs, 1974).

(c) Outer ring structures. Although spiral patterns have attracted more attention, ring structures are perhaps equally common both in the central regions of lenticulars and spirals of the $(r)$ variety (Randers, 1940; de Vaucouleurs, 1959b; Sandage, 1961) and around the main body of galaxies of various types. This outer $(R)$ structure is often less conspicuous because of its lower surface brightness but it is not rare; it is especially frequent in late lenticulars and early type spirals, where it is observed in about 10 to $20 \%$ of these systems. Often a pseudo ring is formed by the faint extensions of the outer arms which are, therefore, not at all branches of 'spirals' but rather arcs of circles or ellipses. Plausible mechanisms for the trapping of particles in near-circular quasi-periodic orbits around a barred stellar system have been discussed by Danby (1965) and especially by Freeman $¥ \ddagger$ (de Vaucouleurs and Freeman, 1972).

\section{Intrinsic Laws of Luminosity Distribution in Normal Galaxies}

Detailed photographic or photoelectric surface photometry in one or two colours is now available for some 100 bright galaxies. Recent studies of this material, supplemented by integral photoelectric photometry of about 1000 systems in two or three colours (de Vaucouleurs, 1961; de Vaucouleurs and de Vaucouleurs, 1972), confirm the subjective impression from photographs (Sandage, 1961) that the luminosity distribution in normal galaxies may be resolved into two major components:

\footnotetext{
¥ Interacting or colliding systems form an interesting subject of study (Vorontsov-Velyaminov, 1959; Arp, 1966), but are not new types of galaxies. (After a collision a car is a wreck, not a new type of car!) Computer simulations (Toomre and Toomre, 1972) demonstrate that tidal distortions can produce many of the curious forms observed in interacting galaxies.

$\$$ The frequency of outer ring structures observed among Seyfert galaxies (three cases: NGC 1068, 4151 and 7469 out of a dozen Seyfert objects in the BGC) does not significantly exceed that in the general population in the same range of types. It seems unlikely that such ring structures, which are resolvable into stars in the nearer examples (Sandage, 1961), might be somehow induced by the transient nuclear activity.
} 
a
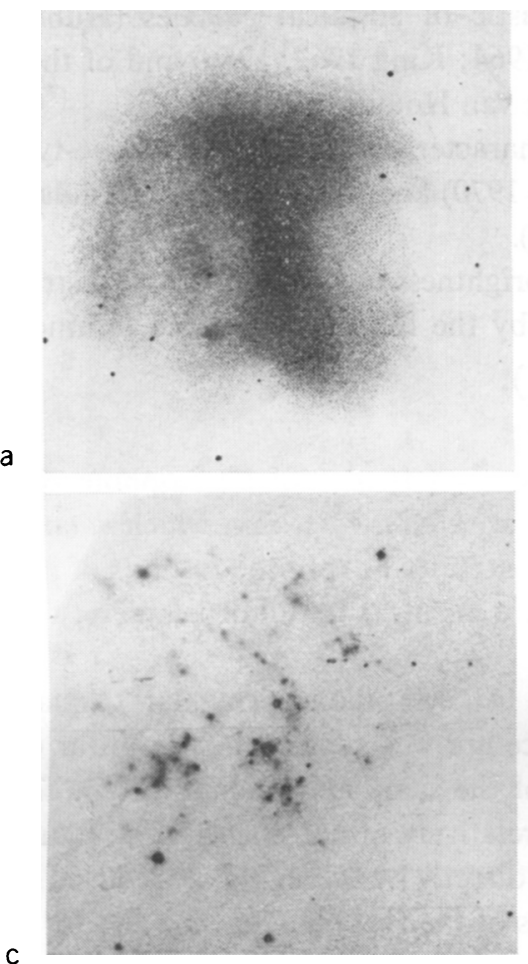
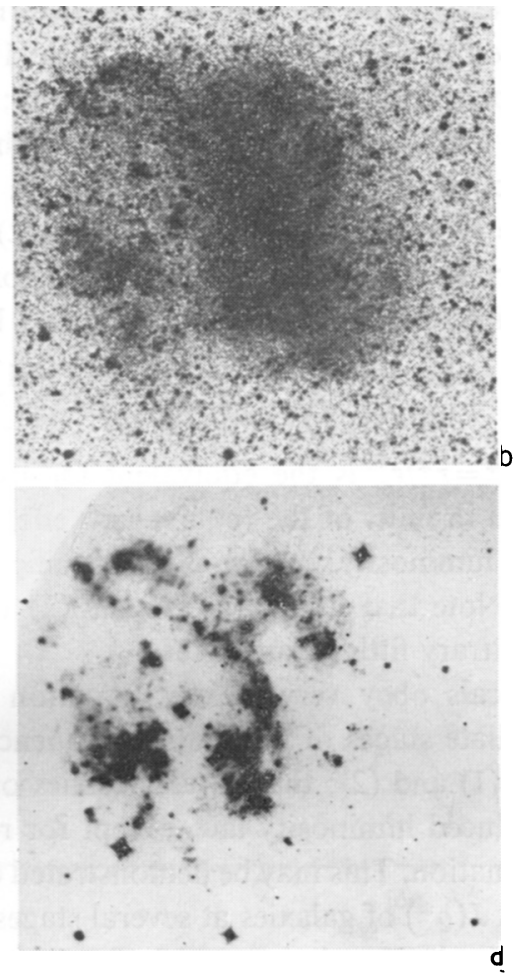

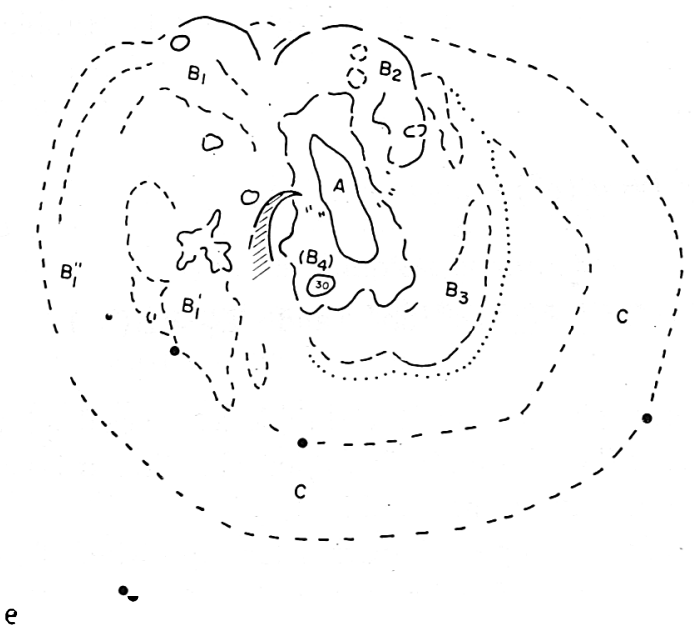

Fig. 2. The Large Magellanic Cloud illustrates the $\gamma$-shaped appearance of SBm galaxies: (a) naked-eye view, after J. Herschel (1847); (b) visible light photograph from Mount Stromlo, 1953; (c, d) ultraviolet photographs by C. Young from the Moon, April 22, 1972 (courtesy Th. Page, NASA Apollo 16 mission), (c) $\lambda \lambda$ 1050-1550 (including $\mathrm{L} \alpha$ ), exposure $1 \mathrm{~min}$; (d) $\lambda \lambda$ 1230-1550 (excluding $\mathrm{L} \alpha$ ), exposure $10 \mathrm{~min}$; (e) key to Cloud structure (de Vaucouleurs and Freeman, 1972). 
(I) a spheroidal component characteristic of elliptical galaxies (Hubble, 1930; de Vaucouleurs, 1948, 1953, 1962; Fish, 1964; King 1962, 1966) and of the bulge of early type spirals (de Vaucouleurs, 1958a; van Houten, 1961);

(II) a flat or exponential component characteristic of the disk of late-type spirals (de Vaucouleurs, 1958a, 1959b; Freeman, 1970) and of Magellanic irregulars (Ables, 1971 ; de Vaucouleurs and Freeman, 1972).

In normalized units the radial surface brightness (specific intensity) distribution in face-on systems is closely approximated by the following reduced luminosity laws

$$
\begin{aligned}
& \log J_{1}\left(\varrho^{*}\right)=-3.33\left(\varrho^{* 1 / 4}-1\right), \\
& \log J_{2}\left(\varrho^{*}\right)=-0.729\left(\varrho^{*}-1\right),
\end{aligned}
$$

where $\varrho^{*}=r^{*} / r_{\mathrm{e}}^{*}$ is the equivalent radius $r_{\mathrm{e}}^{*}=(A / \pi)^{1 / 2}$ of an isophote of area $A$, expressed in units of the (equivalent) effective radius $r_{\mathrm{e}}^{*}$ within which is emitted half the total luminosity; $J=I / I_{\mathrm{e}}$ is the specific intensity $I$ expressed in units of its value at $r^{*}=r_{\mathrm{e}}^{*}$. Note that Equations (1) and (2) are intrinsic and dimensionless; there is no free, arbitrary fitting parameter.

Ellipticals obey very closely Equation (1), Magellanic irregulars Equation (2); intermediate stages of the Hubble sequence are well represented by linear combinations of (1) and (2); that is, all galaxies of the same morphological type follow the same reduced luminosity law, except for relatively minor effects of structural detail and inclination. This may be demonstrated directly by averaging the reduced luminosity curves $J\left(\varrho^{*}\right)$ of galaxies at several stages of the Hubble sequence and by matching the average luminosity curves for each stage $t$ by model calculations with two free parameters, being the ratios of the scale factors $\left(I_{\mathrm{e}}\right.$ or $\left.r_{\mathrm{e}}^{*}\right)$ of the two components (I) and (II) which vary more or less continuously along the Hubble sequence. It can also be verified indirectly on the integrated luminosity functions $\Delta m\left(\varrho^{*}\right)$ (Figure 3) normalized to total luminosity $L_{\mathrm{T}}$ and effective radius, that is, in magnitudes

where

$$
\Delta m\left(\varrho^{*}\right)=m\left(\varrho^{*}\right)-m_{\mathrm{T}}=-2.5 \log \frac{L\left(\varrho^{*}\right)}{L_{\mathrm{T}}},
$$

$$
L\left(\varrho^{*}\right)=2 \pi \int_{0}^{\varrho^{*}} I\left(\varrho^{*}\right) \varrho^{*} \cdot \mathrm{d} \varrho^{*} \quad \text { and } \quad L_{\mathrm{T}}=L(\infty) .
$$

The curves for successive stages of the Hubble sequence display a smooth transition from type I (E) to type II (Im) and, in first approximation at least, form a one-parameter family depending only on the Hubble stage.

(a) Sersic's formula. A simple one-parameter expression of the overall luminosity distribution laws in galaxies of different types has been given by Sersic (1968b):

$$
S(m)=k\left(m-m^{\prime \prime}\right)^{n}
$$

where $m^{\prime \prime}=m_{0}-1.086 n$, and $m=-2.5 \log \left[0.921 \int_{0}^{m} I \cdot S(m) \mathrm{d} m\right]$ is the integrated magnitude within the isophote of surface area $S ; m_{0}$ is the surface magnitude at the centre, perhaps excluding a semi-stellar nucleus. The fitting parameter $n$ varies smoothly with morphological type from $n=8$ at type E to $n=2$ at Im. Sersic's formula 


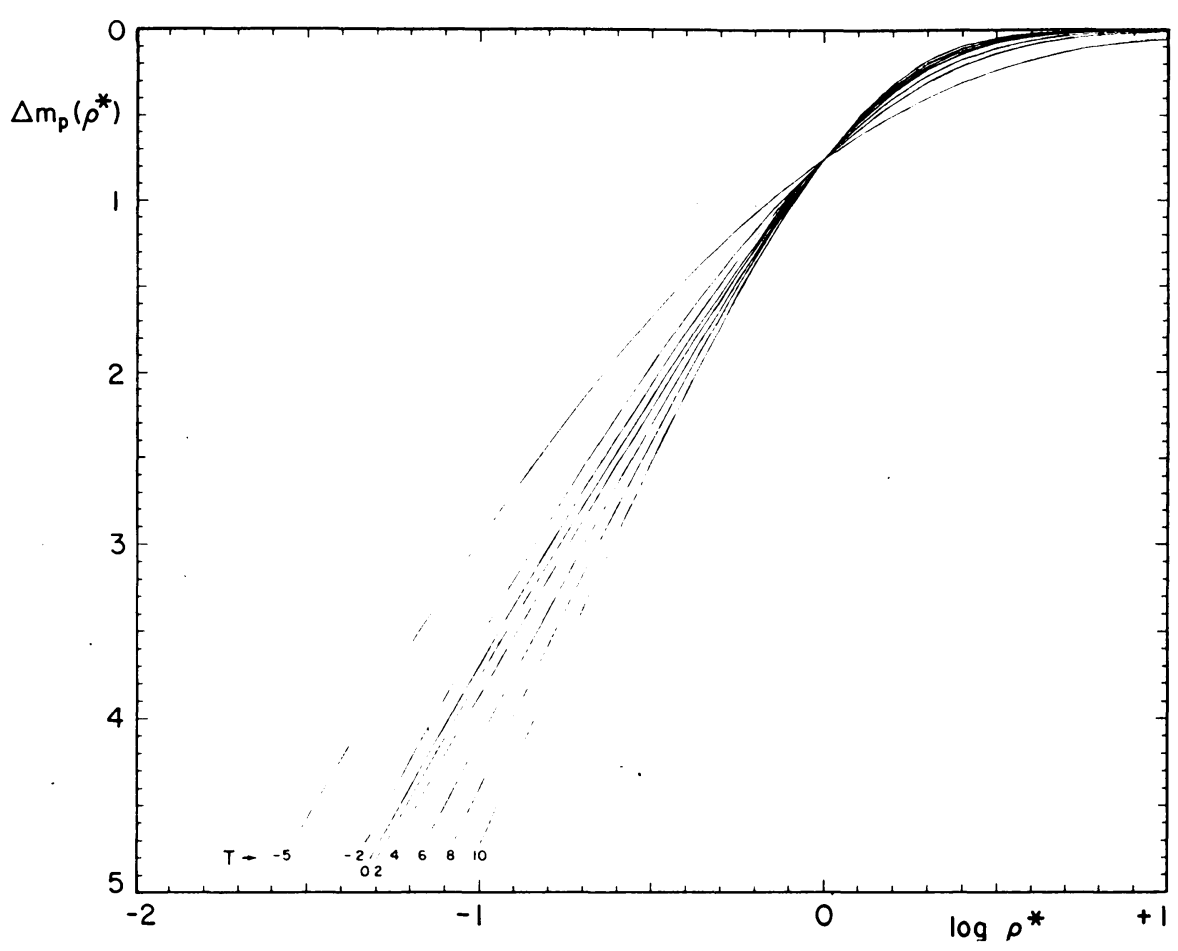

Fig. 3. Normalized integrated luminosity distributions in galaxies as a function of stage $t$ along Hubble sequence. The reduced integrated magnitude curves $\Delta m\left(Q^{*}\right)$, (normalized to $L_{\mathrm{T}}=1$ ), vs reduced equivalent radius (normalized to $r^{*}=1$ ) form a one-parameter family depending on stage $t$.

is more general than either Equation (1) or (2) and includes them as special cases for $n=8$ and 2 , respectively. However, it does not define the characteristic scale factors as directly as (1) and (2) (For further comparisons between the Mt. Stromlo and Cordoba photometric parameters see de Vaucouleurs and Agüero (1973)).

(b) Concentration indices. Perhaps the simplest quantitative measure of the gross structural properties of a stellar system is a light concentration index. Such indices may be derived from the relative integrated luminosity curve $k\left(\varrho^{*}\right)=L\left(\varrho^{*}\right) / L_{\mathrm{T}}$ giving the fraction of the total luminosity emitted within a given radius. In practice two indices have been used (de Vaucouleurs, 1962; Fraser, 1972; de Vaucouleurs and Agüero, 1973), namely those corresponding to the quartiles $k\left(\varrho_{1}^{*}\right)=\frac{1}{4}$ and $k\left(\varrho_{3}^{*}\right)=\frac{3}{4}$ of the integrated luminosity distribution function, that is

$$
C_{21}=r_{\mathrm{e}}^{*} / r_{1}^{*} \text { and } C_{32}=r_{3}^{*} / r_{\mathrm{e}}^{*} \text {. }
$$

Note that $k\left(r_{\mathrm{e}}^{*}\right)=\frac{1}{2}$ by definition.

Plots of $C_{21}$ and $C_{32}$ vs morphological type $T$ (Figure 4) display a smooth transition from early to late galaxy types and are consistent with expected values if the luminosity distribution in galaxies can be resolved into two components, one following the $r^{1 / 4}$ law (1), and the other obeying the exponential law (2). For a pure $r^{1 / 4}$ distribution the 


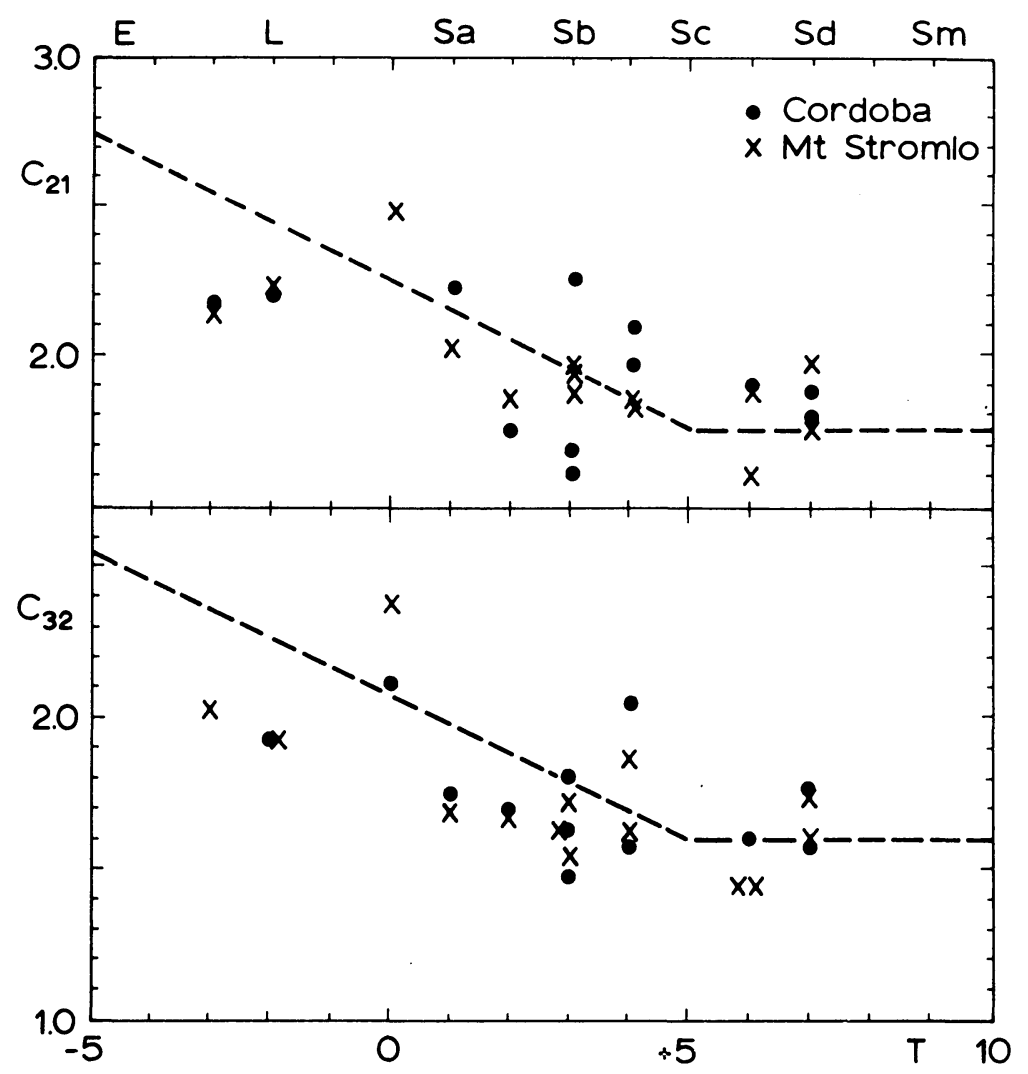

Fig. 4. Concentration indices versus stage along Hubble sequence. The trend reflects transition from a pure spheroidal distribution in ellipticals $(t=-5)$ to a pure exponential distribution in late type spirals $(t \geqslant 6)$ (de Vaucouleurs and Aguero, 1973).

calculated values are $C_{21}=2.74, C_{32}=2.55$ and for a pure exponential distribution $C_{21}=1.75, C_{32}=1.61$. It is clear, however, from Figure 4 that the observational scatter is rather large and, consequently, that concentration indices are not good indicators of morphological types nor acceptable substitutes for the Hubble type.

(c) Tidally truncated dwarf ellipticals: King's formula. Elliptical galaxies in gravitational interaction with a nearby system, in particular dwarf satellites of giant galaxies, appear to be tidally truncated, a situation similar to that of globular clusters in our Galaxy (von Hoerner, 1957; King, 1962). For such systems King $(1962,1966)$ has shown that the projected star density (and by extension the luminosity) distribution is well represented by the semi-empirical two-parameter expression

$$
f=k\left[\frac{1}{\sqrt{1+\left(r / r_{\mathrm{c}}\right)^{2}}}-\frac{1}{\sqrt{1+\left(r_{\mathrm{t}} / r_{\mathrm{c}}\right)^{2}}}\right]^{2},
$$

where $r_{\mathrm{c}}$ is a 'core' radius and $r_{\mathrm{t}}$ a 'tidal' radius or, in other words, a scale factor $\left(r_{\mathrm{c}}\right)$ and a free fitting parameter $\left(r_{\mathrm{t}} / r_{\mathrm{c}}\right)$. This expression gives a fortiori a good representa- 
tion of the luminosity distribution in isolated ellipticals for a sufficiently large value of $r_{\mathrm{t}} / r_{\mathrm{c}}$, when Equation (6) and (1) are numerically in close agreement (King, 1966). King's formula has been extensively used in analyses of the low density Sculptor-type satellites of our Galaxy which do not obey Equation (1) at all (Hodge, 1961; de Vaucouleurs and Ables, 1968; Hodge and Michie, 1969; Karachentseva, 1972).

A second type of truncated dwarf more recently identified is the high-density, compact type, for example NGC 4486B, the compact companion of M87 in the Virgo cluster (Rood, 1965; Faber, 1973) and NGC 5846A, the satellite of NGC 5846 in the NGC 5850 group (de Vaucouleurs, 1960; King and Kiser, 1973). Unlike the lowdensity dwarfs these high density systems obey Equation (1) quite well up to the centre, but suffer a sudden drop-off near the tidal limit as per Equation (6). ${ }^{\ddagger}$

(d) D galaxies. As defined by Morgan (Mathews et al., 1964) these are supergiant galaxies with an elliptical-like core in an extensive lenticular-like envelope. Variants or transition types are denoted as ED, DE and cD. Many coincide with core-halo radio sources in rich clusters, e.g. NGC 6166, classified cD. Others are isolated or in small groups, e.g. NGC 1316 (D) and 5128 (DE). Detailed photometry of several typical objects (Sersic, 1957; de Vaucouleurs, 1969, G. and A. de Vaucouleurs, 1970; Arp and Bertola, 1969, 1971) confirms Morgan's description and demonstrates quantitatively that the luminosity profiles can be resolved neatly into a spheroidal core obeying Equation (1) and an exponential corona obeying Equation (2), the latter with an unusually large scale factor $(\sim 100 \mathrm{kpc})$ and an abnormally low brightness factor.

Typically, for instance in M87, one-third of the total luminosity arises from the central spheroidal component and two-thirds from the corona. Such coronas contribute to - or perhaps are the sources of - the intergalactic luminosity observed in several rich galaxy clusters (de Vaucouleurs and de Vaucouleurs, 1970; Welsh and Sastry, 1971; Oemler, 1973). These diffuse optical sources are apparently co-extensive with the large radio and X-ray sources that have been detected in some clusters (Gursky et al., 1971; Kellogg et al., 1971; Owen, 1973) on a scale of order $\sim 1 \mathrm{Mpc}$, but there is no evidence in the colour data that the optical source is of non-thermal origin; in particular the metallicity index $Q$ agrees well with that of metal-poor globular clusters and Sculptor-type dwarf ellipticals.

\section{True and Maximum Ellipticities of Galaxies}

In principle the frequency function of true ellipticities $\left(e=1-c / a=1-q_{0}\right)$ of spheroidal galaxies can be derived easily from the observed frequency function of apparent ellipticities $(\varepsilon=1-b / a=1-q)$ under the assumption of random orientation of the

$\ddagger$ Because of the very small angular scale of these compact objects $\left(r^{*}{ }_{e}=5 " 0\right.$ for NGC 4486B, de Vaucouleurs and Fraser, unpubl.) two-dimensional deconvolution of the raw photometry is essential to derive correctly the true luminosity distribution. Some early statements on the structure of NGC 4486B (Minkowski, 1962; Rood, 1965) are incorrect because of insufficient allowance for seeing effects. 
spin axes. This problem has been often treated, mainly with respect to elliptical and lenticular galaxies and most recently by Sandage et al. (1970) from statistics of the BGC data. (See de Vaucouleurs, 1959b for a review of earlier work and references.)

Recently we have critically re-analyzed this problem on the basis of new and better data on the axis ratios of isophotal diameters of over 2000 galaxies. The data, if not quite complete for a given volume of space, were restricted to a limit of corrected face-on apparent diameters down to which the catalogue is $\sim 50 \%$ complete.

Diameters refer now to a definite, photometrically calibrated isophote $\left(\mu_{B}=\right.$ $=25.0 \mathrm{mag}(\operatorname{arcsec})^{-2}$ ) and, in comparisons between observed and calculated distributions, proper allowance was made for the effects of accidental measuring errors in $b / a$. Results are illustrated in Figures 5 and 6. The most significant conclusions are:

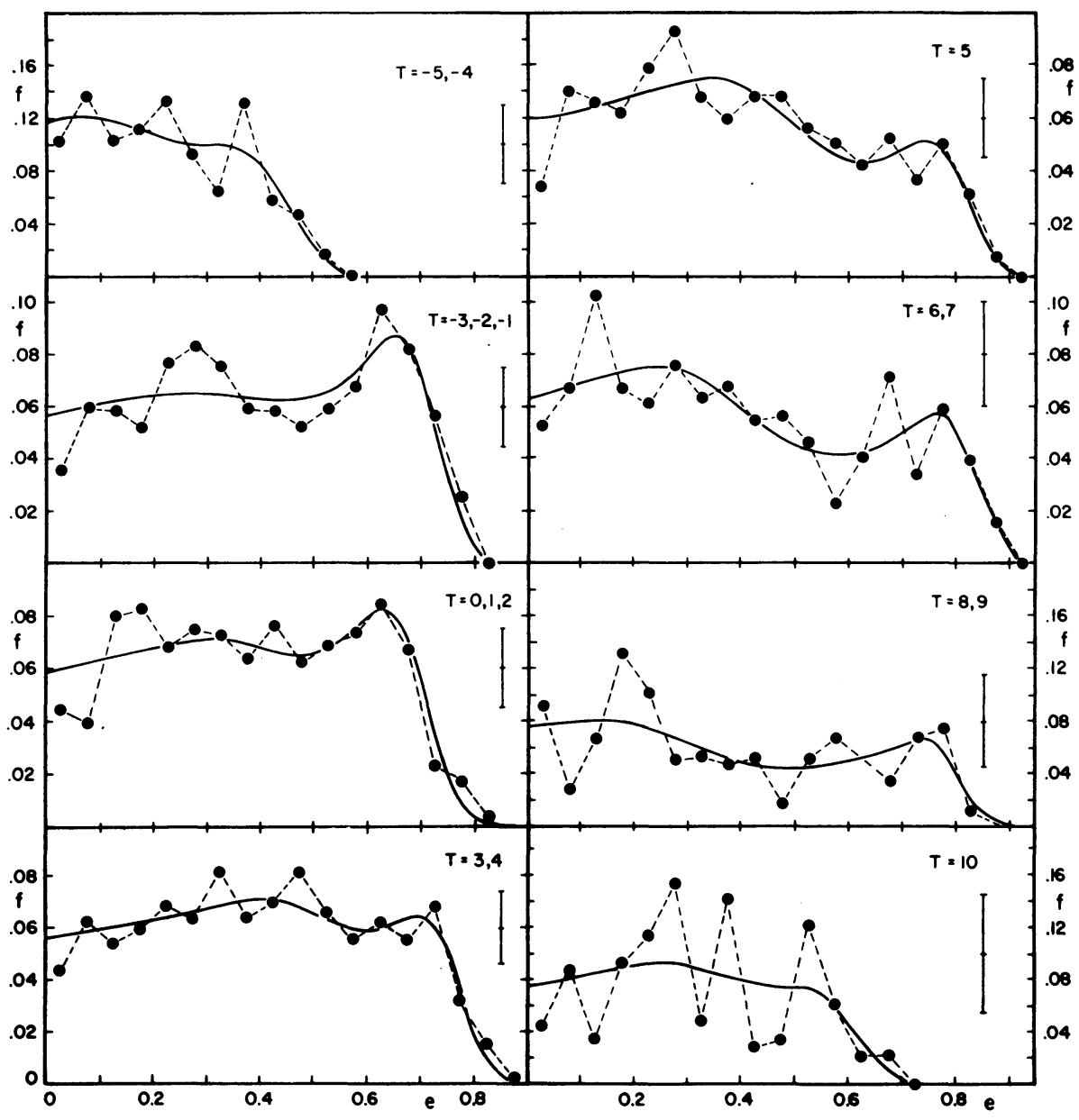

Fig. 5. Relative frequencies of apparent ellipticities among galaxies having face-on isophotal diameters $D(0)_{25} \gtrsim 2^{\prime}$. Curves are calculated frequencies for 2-component Gaussian models. Error bars $(2 \sigma)$ are expected random fluctuations for average count per 0.05 interval in $e$. 


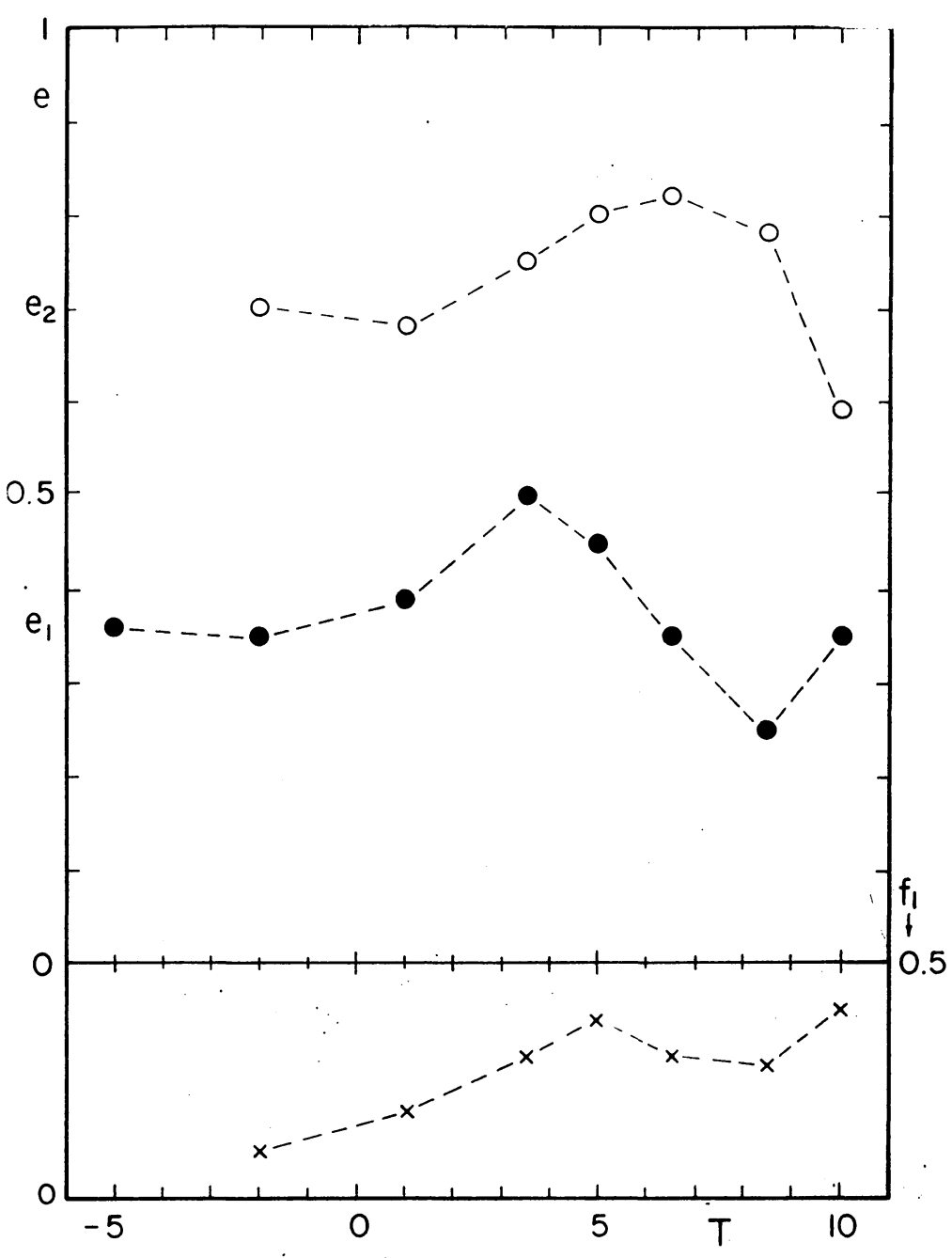

Fig. 6. Mean ellipticities of Gaussian component models of ellipticity distribution functions vs. stage along Hubble sequence. Ellipticity of flatter population component is maximum near type Sd $(t=7)$. At bottom: relative frequency $f_{1}$ of less flat component whose $\left\langle e_{1}\right\rangle$ is near that of ellipticals $(t=-5)$.

(a) the distribution of true ellipticities among $\mathrm{E}$ galaxies is definitely not uniform up to $\sim \frac{2}{3}\left(q_{0} \simeq \frac{1}{3}\right)$, in agreement with the conclusions of Sandage et al. (1970). The best fitting single gaussian model has $\langle e\rangle \simeq 0.36$ (i.e. E 6.5) with small cosmic dispersion $\sigma_{\mathrm{e}} \simeq 0.1$. Spherical galaxies are rare or absent.

(b) Lenticular galaxies, as is well known, tend to be more flattened than ellipticals, but the analysis suggests that two components are present: a major group $(\sim 90 \%$ of sample) with $\langle e\rangle=0.65$ and $\sigma_{\mathrm{e}} \simeq 0.1$, and a smaller group $(10 \%)$ with $\langle e\rangle \simeq 0.35$ and $\sigma_{\mathrm{e}} \simeq 0.05$. 
(c) Spiral galaxies from S0/a to Sm have ellipticity functions very similar to the lenticulars' with a major group $(\sim 70 \%$ of sample) with $\langle e\rangle=0.7$ to 0.8 and, again, a minor one $(\sim 30 \%)$ with $\langle e\rangle \simeq 0.4$. These two components may be tentatively identified with the two classes of spirals having small bulges and large bulges respectively.

(d) Magellanic irregulars Im may also form two groups, but more nearly balanced with $\langle e\rangle \simeq 0.6(60 \%)$ and $\langle e\rangle \simeq 0.35(40 \%)$.

Figure 6 confirms the results of an earlier study of the Reference Catalogue data (Heidmann et al., 1971) showing that $q_{0}=c / a$ decreases steadily and smoothly along the Hubble sequence from $\mathrm{E}(t=-5)$ to $\mathrm{Sd}(t=7)$ where it reaches a minimum. The flattest galaxies are observed at this stage, for example IC 2233 (Figure 7) which has an isophotal axis ratio of almost 10 to $1 \ddagger$. This property is consistent with our previous

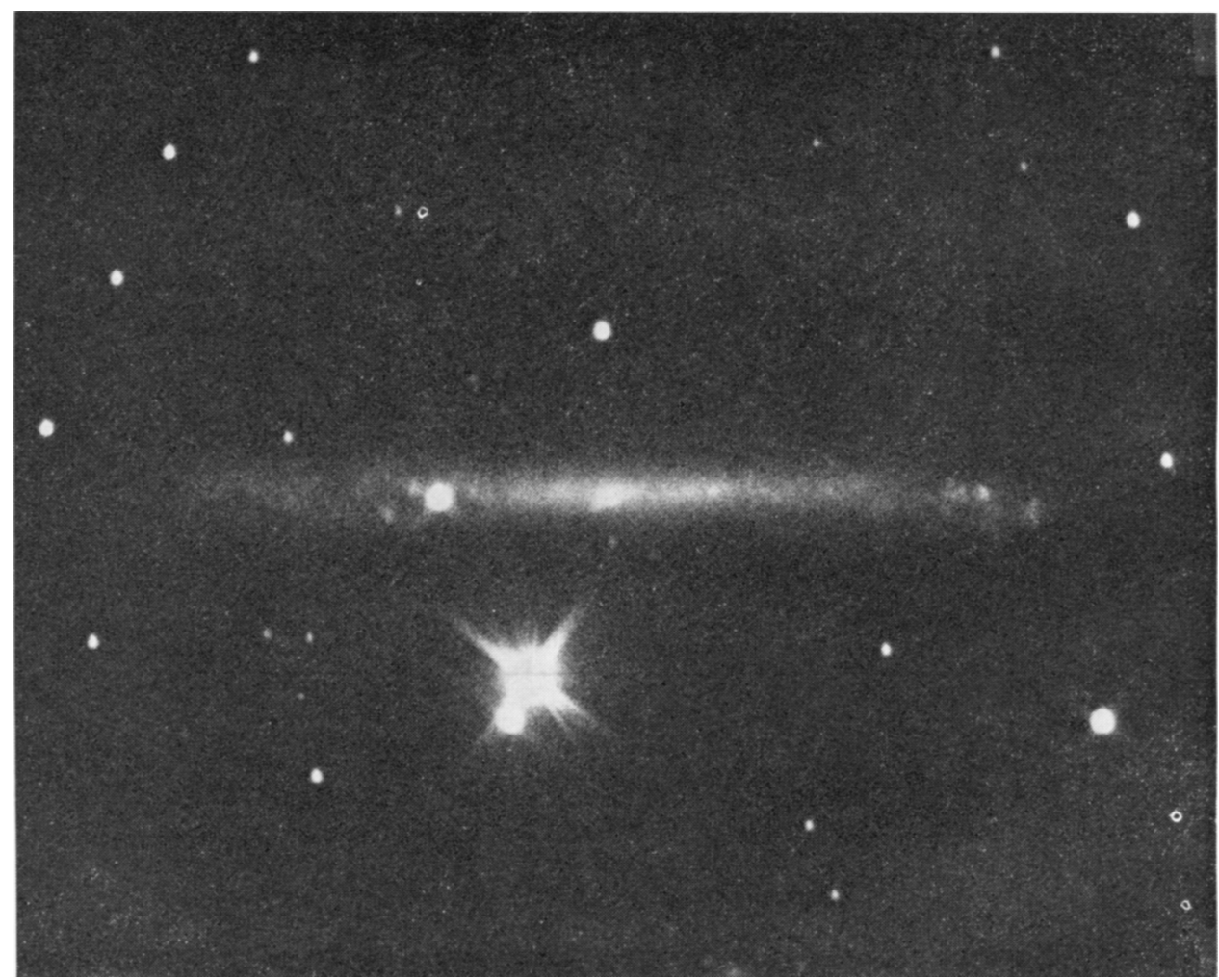

Fig. 7. One of the flattest galaxies, IC 2233, an Sd system seen almost exactly edge-on, has no perceptible spheroidal component, only an extremely thin exponential disk. (McDonald Observatory, Struve 205-cm reflector, March 20, 1969; IIa-0, $61 \mathrm{~min}$ ).

\$ Similar and even more extreme examples are given by Vorontsov-Velyaminov (1967), but the axis ratios quoted (1/25 for MCG 7-30-11, 1/35 for MCG 4-9-60) are probably exaggerated by the wellknown measuring errors first discussed by Holmberg (1946) and corrected in the BCG (de Vaucouleurs and de Vaucouleurs, 1964). 
conclusion from photometry that the ratio of the two components (spheroidal and flat) of the luminosity distribution varies smoothly as a function of stage $t$ and it suggests that the Hubble sequence from $\mathrm{Sa}$ to $\mathrm{Sd}$ is basically an angular momentum sequence (Section 8). Beyond stage Sd the ellipticity decreases rapidly so that Magellanic spirals $(\mathrm{Sm})$ have typical axis ratios $q_{0} \simeq 0.2$, and at the chaotic stage (Im) $q_{0} \simeq 0.4$; nevertheless, contrary to early ideas, irregulars are by no means spherical (de Vaucouleurs, 1970a; Hodge and Hitchcock, 1966); most, perhaps all, are clearly rotating although more slowly than earlier types (Section 8). Examples of edge-on Magellanic systems such as NGC 55, 1507, 2188, etc. are given and illustrated by de Vaucouleurs and Freeman (1972).

\section{Characteristic Parameters of Spheroidal Systems}

There are not yet enough reliable photometric data to derive the mean values and distribution functions of the characteristic linear scale and density factors $\left(r_{\mathrm{e}}, I_{\mathrm{e}}\right)$ of spheroidal systems or of the spheroidal component of mixed systems. Only isolated examples are known for some typical giant, intermediate and dwarf systems (Table III).

TABLE III

Effective parameters of spheroidal systems

\begin{tabular}{|c|c|c|c|c|c|c|c|c|c|}
\hline Galaxy & $m-M$ & $\begin{array}{l}\Delta \\
(\mathrm{Mpc})\end{array}$ & $\begin{array}{l}2 r^{*} \mathrm{e} \\
(\operatorname{arc} \min )\end{array}$ & $\begin{array}{l}D_{\mathrm{e}} \\
(\mathrm{kpc})\end{array}$ & $m_{\mathrm{T}}(B)$ & $M_{\mathrm{T}}(B)$ & $\begin{array}{l}\mu_{\mathrm{e}}^{\prime}(B) \\
(\operatorname{arcsec})^{-2}\end{array}$ & $\begin{array}{l}L_{\mathrm{e}}^{\prime} \\
\odot \mathrm{pc}^{-2}\end{array}$ & Notes \\
\hline N221 & 24.7 & 0.7 & 1.5 & 0.30 & 8.96 & -15.74 & 19.22 & 1600 & \\
\hline N4486B & $30.5:^{b}$ & $12.5 ?$ & $0.16_{5}$ & 0.60 & 14.28 & -16.22 & 19.72 & 1000 & \\
\hline N4494 & 29.75 & 8.0 & 1.8 & 4.2 & 10.64 & -19.11 & 21.29 & 230 & \\
\hline N3379 & 29.50 & 7.2 & 2.0 & 4.1 & 10.25 & -19.25 & 21.13 & 270 & \\
\hline N224 & 24.7 & 0.7 & 27. & 5.5 & 4.91 & -19.79 & 21.45 & 200 & $\mathrm{a}$ \\
\hline N4649 & 30.5: & $12.5 ?$ & 2.5 & S.1 & 9.73 & -20.77 & 21.09 & 280 & \\
\hline N4486 & 30.5: & $12.5 ?$ & 3.5 & 12.8 & 9.50 & -21.00 & 21.59 & 180 & $\mathbf{a}$ \\
\hline Fornax & 22.0: & 0.2 : & 23. & 1.35 & 9.04 & -13.0 : & 24.8 & 9 & \\
\hline
\end{tabular}

a for spheroidal $\left(r^{1 / 4}\right)$ component only.

$b$ the colons indicate uncertain values.

(a) High density systems have effective diameters ranging from $\sim 0.5 \mathrm{kpc}$ in dwarf, compact E galaxies such as M32 and NGC 4486B, to $\sim 10 \mathrm{kpc}$ in giant systems such as NGC 4649 and the spheroidal components of M31 and NGC 4486, with a ratio of 20 to 1 .

The specific intensity $I_{\mathrm{e}}$ at $r_{\mathrm{e}}\left(\mu_{\mathrm{e}}\right.$ in mag per unit solid angle) is related to the average surface brightness $\mu_{\mathrm{e}}^{\prime}$ within $r_{\mathrm{e}}$; for an $r^{1 / 4}$ distribution $\mu_{\mathrm{e}}^{\prime}=\mu_{\mathrm{e}}-1.40$. Values of $\mu_{\mathrm{e}}^{\prime}$ in $B$ mag $(\operatorname{arc~sec})^{-2}$ and in solar units per square parsec are listed in Table III. The range for these dense systems is from $\sim 19.5$ to $\sim 21.5 \mathrm{mag}(\operatorname{arc~sec})^{-2}(\sim 800$ to $\sim 200$ suns $\mathrm{pc}^{-2}$ ), with a ratio of only 4 to 1 .

In the denser systems the $r^{1 / 4}$ law apparently applies right up to the centre where, according to Equation (1), the surface brightness should be $2.5 \times 3.33=8.3 \mathrm{mag}$ 
brighter than at $r_{\mathrm{e}}$; that is $\mu_{0}=\mu_{\mathrm{e}}-8.3=\mu_{\mathrm{e}}^{\prime}-6.9$. For example, in the nucleus of M31 the observed luminosity peak, after correction for instrumental smoothing, is $\mu_{0} \simeq 15.0 \mathrm{mag}(\operatorname{arcsec})^{-2}$ (Johnson 1961), or $\sim 6.5 \mathrm{mag}$ brighter than $\mu_{\mathrm{e}}^{\prime}$, in close agreement with the $r^{1 / 4}$ formula. The situation is about the same for M32 where, as in $\mathrm{M} 31$, the central luminosity density approaches or possibly exceeds $10^{5} \mathfrak{L}_{\odot} \mathrm{pc}^{-3}$ (de Vaucouleurs, 1953; Kinman, 1965).

(b) Low density dwarf spheroidal systems which do not obey the $r^{1 / 4}$ law may be characterized by their core and tidal radii. Examples have been collected by van den Bergh (1968) and Karachentseva (1972) mainly from star counts by Hodge (1961-65). Typical values are in the range $0.2-0.3 \mathrm{kpc}$ for $r_{\mathrm{c}}$ and $0.8-1.2 \mathrm{kpc}$ for $r_{\mathrm{t}}$, at least for the dwarf satellites of our Galaxy. Photometric effective radii are not yet known for these objects, except for Fornax where $r_{\mathrm{e}}^{*}=11.5=0.7 \mathrm{kpc}$ (de Vaucouleurs and Ables, 1968); for comparison $r_{\mathrm{c}}=0.9 \mathrm{kpc}$ and $r_{\mathrm{t}}=3.1 \mathrm{kpc}$ from star counts (Hodge, 1961).

The light concentration in low density dwarfs is slight and the central area with radius $r \simeq r_{\mathrm{e}}^{*} / 2$ has a nearly constant surface brightness; for example in Fornax the maximum reaches only $\mu_{0}=24.6 \mathrm{mag}(\operatorname{arcsec})^{-2}=11 \mathfrak{I}_{\odot} \mathrm{pc}^{-2}$, while at $r=r_{\mathrm{e}}^{*}=$ $=10^{\prime}=0.65 \mathrm{kpc}, \mu_{\mathrm{e}}=25.2 \mathrm{mag}(\operatorname{arc~sec})^{-2}$ and the average between $r=0$ and $r=r_{\mathrm{e}}^{*}$ is $\mu_{\mathrm{e}}^{\prime}=24.8 \mathrm{mag}(\operatorname{arcsec})^{-2}=9 \mathfrak{L}_{\odot} \mathrm{pc}^{-2}$ (de Vaucouleurs and Ables, 1968; Hodge, 1973 ) or $1 \%$ of the corresponding light density in M32. Preliminary photometry of Sculptor and other nearby dwarfs indicates even lower densities, $\mu_{0}>25 \mathrm{mag}(\operatorname{arcsec})^{-2}$ and down to the current detection threshold for such objects $\left(\mu_{0} \simeq 27 \mathrm{mag}(\operatorname{arcsec})^{-2} \simeq\right.$ $\simeq 1 \mathfrak{L}_{\odot} \mathrm{pc}^{-2}$ ) which is $10^{-4}$ to $10^{-5}$ of the central density of M32. Large numbers of dwarfs with such densities or lower ones might remain undetectable by current techniques.

\section{Characteristic Parameters of Flat Systems}

Although photometric information is still very incomplete, statistical studies of available data (Freeman, 1970; Heidmann et al., 1971) lead to rather remarkable general conclusions. As noted in Section 3 the flat (disk) components of lenticulars and spirals follow closely the exponential luminosity law (2) which may also be written as

$$
I(r)=I_{0} \exp (-\alpha r),
$$

where $\alpha$ is the inverse of the scale length $\Lambda$; it is measured by the photometric gradient $G(r)=\mathrm{d}(\log I) / \mathrm{d} r$ since $\Lambda=-0.4343 / G(r)$. For a pure exponential distribution the relation between effective radius $r_{\mathrm{e}}$ and scale length $\Lambda$ is $r_{\mathrm{e}}=1.6785 \Lambda$, and the relation between central and effective intensities is $I_{0}=0.729 I_{\mathrm{e}}$ or, in magnitudes, $\mu(0)=$ $=\mu_{\mathrm{e}}-1.82$. Similarly, the average surface brightness within $r_{\mathrm{e}}$ is $\mu_{\mathrm{e}}^{\prime}=\mu_{\mathrm{e}}-0.70=$ $=\mu(0)+1.12$.

Freeman (1970) has collected values of $\mu(0)$ and $\Lambda$ for 36 disk galaxies having welldefined exponential components and small enough inclination angles that the corresponding values of $\mu(0)_{\mathrm{c}}$, corrected to $i=0$ and to zero galactic absorption, could be reasonably calculated. A number of correlations with morphological types emerge 
from this sample; in particular

(1) $\Lambda$ has a range of about 10 to 1 , from $0.5 \mathrm{kpc}$ to $\sim 5 \mathrm{kpc}$ for types earlier than $\mathrm{Sc}$;

(2) the maximum value of $\Lambda$ decreases from $\sim 5 \mathrm{kpc}$ at $\mathrm{Sc}(t=5)$ to $\sim 1 \mathrm{kpc}$ at Im $(t=10)$, confirming earlier results on the dependence of galaxy 'diameters' on Hubble type (cf. Holmberg, 1950; de Vaucouleurs, 1959b, p. 314-5);

(3) for some three-quarters of the sample (28 out of 36$), \mu(0)_{c}$ varies little from $\left\langle\mu(0)_{\mathrm{c}}\right\rangle=21.65 \mathrm{mag}(\operatorname{arc~sec})^{-2}(\sigma \simeq 0.3 \mathrm{mag})$ and is independent of morphological type from $L^{-}(t=-3)$ to $\operatorname{Im}(t=10)$ (Figure 8$)$. However, some individual values depart from this mean by up to +2 mag (IC 1613) and -3 mag (NGC 5236).

Since the total luminosity of the exponential disk is $L_{\mathrm{T}}=2 \pi I_{0} \Lambda^{2}$, the result that $I_{0} \simeq$ constant implies that a close correlation must also exist between the total absolute magnitude of the disk and the scale length (Figure 9). This conclusion is in general agreement with, and accounts for the results of, earlier studies demonstrating a rather close luminosity-diameter correlation for spiral galaxies (Holmberg, 1958, 1964; de Vaucouleurs, 1959b; Heidmann, 1967, 1969; Heidmann et al., 1971).

Nevertheless, two questions present themselves:

(1) is $\langle\mu(0)\rangle$ really independent of galaxy type or is it an effect of bias in the selec-

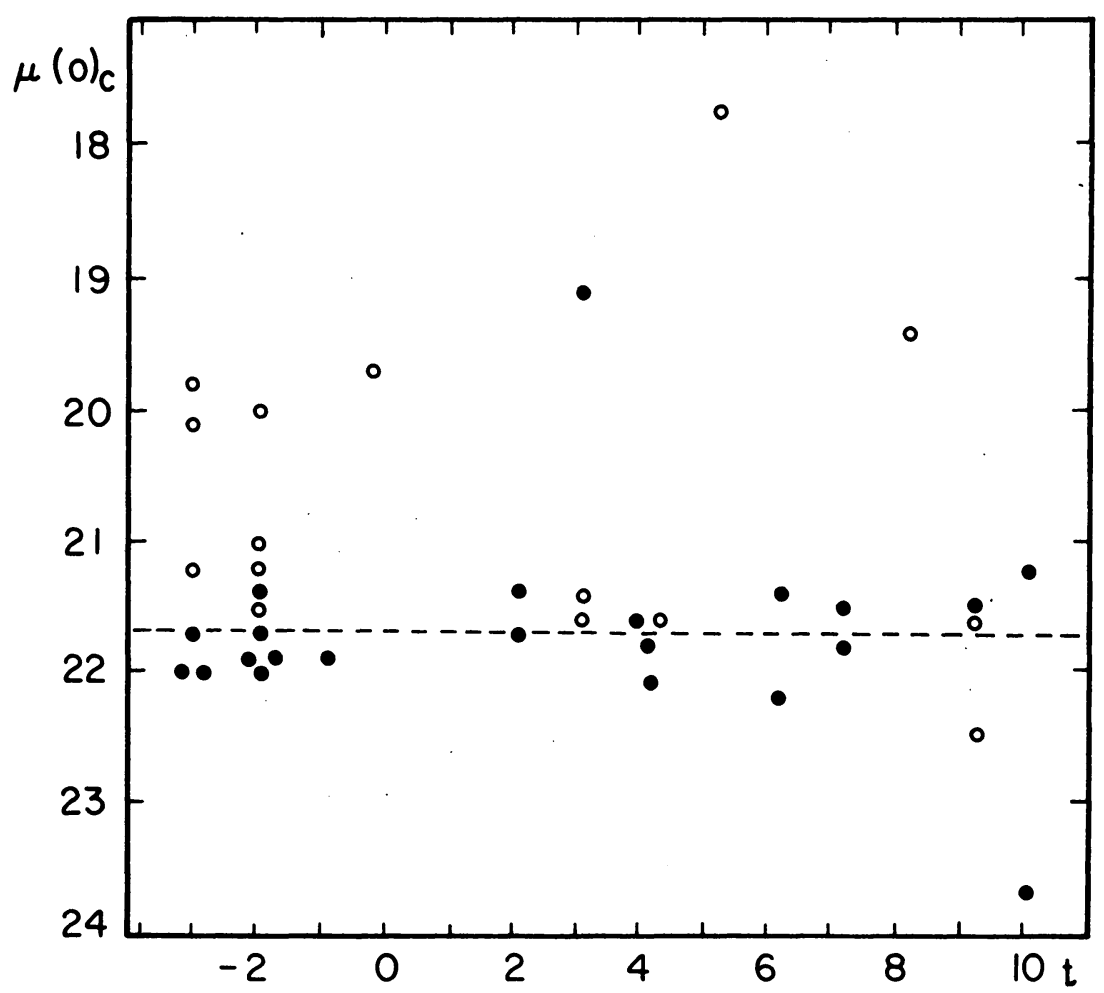

Fig. 8. Corrected face-on central luminosity $\mu(0)_{\mathrm{c}}$ for exponential disks of 35 galaxies vs morphological type, after Freeman (1970). Note that $\left\langle\mu(0)_{c}\right\rangle$ is independent of Hubble type, but some individual galaxies are aberrant. 


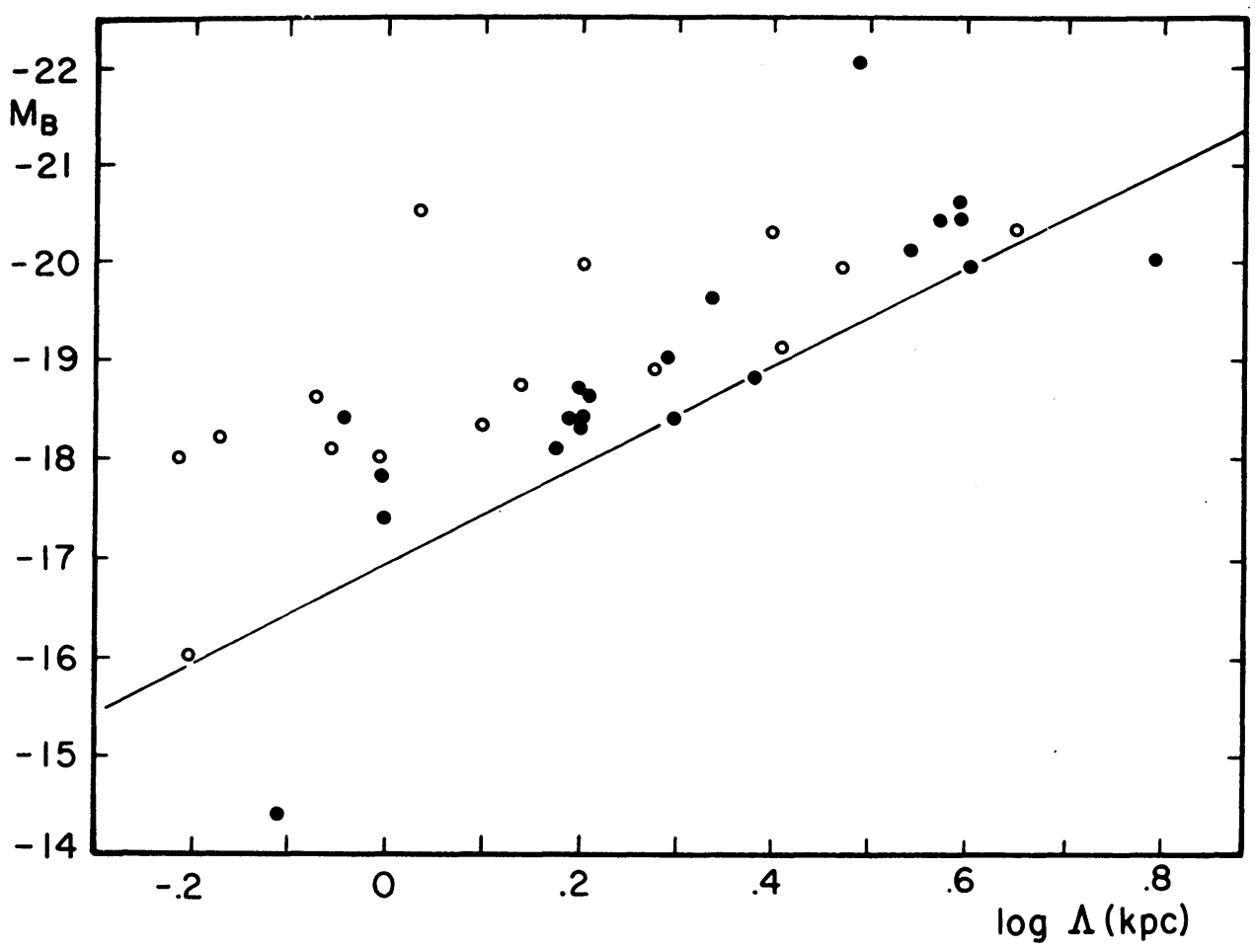

Fig. 9. Correlation between absolute magnitude $M_{\mathrm{B}}$ and length scale $\alpha^{-1}=\Lambda(\mathrm{kpc})$. Straight line is theoretical relation for exponential disk with $B(0)_{\mathrm{c}}=21.65 \mathrm{mag}(\operatorname{arc~sec})^{-2}$.

tion of objects for detailed photometry (there is very little photometry of low-surfacebrightness objects)?

(2) is the dispersion about the mean really as small as $0.30 \mathrm{mag}$ or, again, is it a result of selection effects in a small sample ( 8 objects out of 36 were rejected in the analysis)?

Some recent results suggest that both questions must be answered affirmatively at least for the early and late-type systems:

(1) the discovery of large and very faint coronas around some early-type cD galaxies (Section 3) demonstrates the existence of at least some exponential components as faint as $\mu(0)_{\mathrm{c}} \simeq 24.5 \mathrm{mag}(\operatorname{arc~sec})^{-2}$ (de Vaucouleurs, 1969); the elongated shape of the isophotes (Arp and Bertola, 1969, 1971) suggests that the coronas are flattened;

(2) comparison of several late-type spirals and irregulars with pure exponential distributions (de Vaucouleurs and Freeman, 1972, Fig. 47) indicates among them a range of at least $3.0 \mathrm{mag}$ in $\mu(0)$ from $\sim 20.5$ to $\sim 23.5 \mathrm{mag}(\operatorname{arcsec})^{-2}$. As to other galaxy types, some lenticulars and spirals with very faint disks are known to exist, e.g. NGC 5365 (de Vaucouleurs, 1956a), but no photometry is available for them. 


\section{Characteristic Masses and Densities}

Too little information is available yet to estimate reliably the total mass and density distribution functions of galaxies either in general or of particular types. However, fragmentary results on some typical objects give useful orders of magnitudes.

\subsection{SPHEROIDAL SYSTEMS: VELOCITY DISPERSION}

The classical method assumes that a spheroidal system may be approximated by a spherical distribution of point masses in stable, statistical, dynamical equilibrium with a constant velocity dispersion $\sigma_{v}$. Then, with the further hypothesis that the massdensity distribution in space may be derived from the projected luminosity-density distribution, i.e. $f=\mathfrak{M} / \mathfrak{L}=$ constant, and that the latter obeys the $r^{1 / 4}$ law (Poveda, 1958,1960 ) - or some equivalent distribution - the total mass is given by

$$
\mathfrak{M}_{\mathrm{T}} \simeq 3 r_{\mathrm{e}} \sigma_{\mathrm{v}}^{2} / G
$$

where $\sigma_{v}$ is the central velocity dispersion. It is derived from the observed line-of-sight component by $\sigma_{v}=\lambda \sigma_{\mathrm{r}}$ where $\lambda$ is a factor allowing for the shape of the velocity ellipsoid within the range $1 \leqslant \lambda \leqslant 3$ from linear (radial oscillations) to spherical (isotropic).

Many assumptions and approximations are involved here:

(1) the hypothesis $f=\mathfrak{M} / \mathfrak{L}=$ constant, independent of radius, conflicts with the observation that the colour index is not strictly constant but decreases outwards from the centre;

(2) the proper value of $\lambda$ is uncertain; most authors argue that $2<\lambda<3$ at least near the centre (Poveda et al., 1960; Burbidge et al., 1961);

(3) the basic assumption of sphericity is contradicted by the recent analyses of distribution of ellipticity (Section 4). As shown by King (1961), even a small intrinsic ellipticity $e=1-c / a$ implies a rotational kinetic energy $T$ of the same order as that in random motions $W$, and in a first approximation (homogeneous spheroid) $T /(W+T)=$ $=(8 / 5) e$. Thus for the average true ellipticity of elliptical galaxies $e=0.36$ (Section 4), $T /(W+T) \simeq 0.6$ or $T \simeq 1.5 \mathrm{~W}$, implying a total mass 2.5 times that calculated for a globular non-rotating model (King, 1962).

(4) the assumption $\sigma_{v}=$ constant, independent of $r$, cannot be exact since the only exact equilibrium solution is the isothermal gas sphere which has infinite mass and radius; $\sigma_{v}$ must decrease outwards (Woolley and Robertson, 1956; Mitchie, 1963; King, 1966). Up to now technical difficulties have prevented direct measurement of $\sigma_{\mathrm{r}}(r)$ except at $r \simeq 0$. However, a slight compensation arises through the fact that the line width measured at $r=0$ is a luminosity-weighted average along the line of sight.

(5) the derivation of $\sigma_{\mathrm{r}}$ from the observed line broadening in the galaxy spectrum compared with a stellar spectrum of 'matching' spectral type (Burbidge et al. 1961; Minkowski, 1962; Morton and Chevalier, 1972) is subject to systematic errors; in particular the composite nature of galaxy spectra introduces a spurious broadening which, until recently, had not been evaluated.

(6) Finally, the standard simplifying assumption of a unique stellar mass value 
in the theoretical model needs re-examination even if equipartition is not as pronounced as was once presumed (de Vaucouleurs, 1953; Woolley, 1954).

It is apparent that values commonly quoted for the masses of spheroidal systems (Poveda, 1961; Fish, 1964) are subject to large uncertainties. Some authors have concluded that the only quantities that can be safely inferred are the central density $\varrho(0)$ and mass-luminosity ratio (King and Minkowski, 1972). Recent determinations by photoelectric scanning or television techniques (Morton and Chevalier 1972, 1973; de Vaucouleurs, 1973) using more rigorous model fitting procedures suggest, however, that the older photographic estimates of $\sigma_{\mathrm{r}}(0)$ are often too large for various reasons, in particular the neglect of compositeness in galaxy spectra. Some old and new values of $\sigma_{r}(0)$ are compared in Table IV. The net effect is a significant reduction of previous mass estimates, but more observations by modern techniques are needed to replace all older data.

For the present, provisional conclusions are:

(1) total masses of spheroidal (mainly E) galaxies cover an extremely wide range $\left(10^{5}<\mathfrak{M} / \mathfrak{M}_{\odot}<10^{13}\right)$ from the Local Group 'pygmy' systems to supergiant $\mathrm{cD}$ galaxies (Table V).

TABLE IV

Central radial velocity dispersion $\sigma_{\mathrm{r}}(0)$ in spheroidal systems

\begin{tabular}{llllllll}
\hline NGC & Type & \multicolumn{2}{c}{ Photographic } & \multicolumn{2}{c}{ Photoelectric } \\
& & {$[1]$} & {$[2]$} & {$[3]$} & & {$[4]$} & {$[5]$} \\
221 & -6 & 100 & 96 & - & 60 & 60 \\
224 & +2 & 225 & - & - & 150 & 120 \\
3115 & -3 & 205 & -1 & - & $230^{\text {a }}$ & 215 \\
3379 & -5 & - & 187 & - & 125 & - \\
4486 & -4 & $490:$ & - & 550 & - & - \\
$4486 B$ & -6 & $370:$ & - & - & $160:$ & - \\
4494 & -5 & - & - & - & - & 160 \\
7332 & -2 & - & - & - & - & 160
\end{tabular}

[1] Minkowski 1962.

[2] Burbidge et al. (1961).

[3] Brandt and Roosen (1969).

[4] scanner: de Vaucouleurs (1973).

[5] SEC Vidicon: Morton and Chevalier $(1972,1973)$.

a may be affected by rotation of nucleus.

TABLE V

Masses and mass/luminosity ratios of spheroidal systems

\begin{tabular}{|c|c|c|c|}
\hline Galaxy & Examples & Mass range $\mathrm{a}^{\mathrm{a}}$ & $\mathfrak{M} / \mathfrak{L}^{*}{ }_{B}$ \\
\hline Supergiant $\mathrm{E}$ and $\mathrm{cD}$ & N4486, N4889 & $>10^{12}$ & $>30$ \\
\hline Giant E, bulges Sa-Sb & N224, N3379 & $10^{10}-10^{12}$ & $10-30$ \\
\hline High-density dwarfs & N221, N4486B & $10^{8}-10^{10}$ & $3-10$ \\
\hline Low-density dwarfs & For, Scl & $10^{6}-10^{8}$ & $1-3$ \\
\hline Extreme dwarfs & Dra, UMi & $<10^{6}$ & $\sim 1$ ? \\
\hline
\end{tabular}

a solar units. 
(2) the average mass-luminosity ratio has a much smaller range of variation from $\sim 2$ in low-density dwarfs whose stellar population is similar to that of metal-poor globular clusters (McClure and van den Bergh, 1968; de Vaucouleurs and Ables, 1968) to perhaps $\sim 50$ in high density giants whose spectra and colours have super-metal rich characteristics (Spinrad et al., 1971; van den Bergh, 1972a). The relationship is illustrated in Figure 10, after Einasto and Kaasik (1973). This correlation between $\mathfrak{M} / \mathfrak{L}$ and $\mathfrak{M}$ gives some hope of deriving the mass function from the luminosity function (Section 12).

(3) in giant systems the mass-luminosity ratio decreases outwards from high values, $f_{B} \sim 50$, in the core to low values, $f \sim 3$, in the 'halo' (or corona) which, as judged by its colour, has a metal-poor population similar to that of dwarf ellipticals (Figure 11). Models of spheroidal systems based on this concept agree well with observations (Einasto, 1972a).

\subsection{FLAT SYSTEMS: ROTATION}

In flat systems the $z$ component of the velocity dispersion is small compared with the rotational velocity and contributes little to the total kinetic energy. The mass may be derived from thin disk or flat spheroid approximations by a number of well-known standard methods (Perek, 1950; Kuzmin, 1952; de Vaucouleurs, 1959b; Brandt, 1960; Burbidge et al., 1960, 1963; Rubin et al., 1964).

A large number of mass estimates of spiral galaxies have been derived during the past $20 \mathrm{yr}$ from optical and radio $(21-\mathrm{cm})$ observations of rotational velocities. Results have been collected and analyzed in several recent review papers (VorontsovVelyaminov 1970a, b; Kogure and Toya, 1970). However, many such mass estimates are at best lower limits only; in particular, mass values derived from polynomial fits of rotation curves, by disk models having an equatorial radius equal to the maximum range of optically measured velocities, refer to some unknown fractions of the total masses, since in all cases galaxies can be detected photometrically far beyond the range of spectroscopic data. Radio observations suffer much less from this limitation since $21-\mathrm{cm}$ emission is frequently measurable beyond the range of optical detection; however, radio observations are often hampered by insufficient resolution, except in the largest galaxies. Recently, realistic models consistent with both spectroscopic and photometric data have been developed for a few nearby galaxies (Einasto, 1968-1972a).

Late-type and Magellanic barred spirals SBm with little or no spheroidal component have typical masses of the order of $10^{10} \mathfrak{M} \odot$ and $\mathfrak{M} / \mathfrak{L} \simeq 3$ (de Vaucouleurs and Freeman, 1972); the latter value may be typical of the flat component of spirals. Flat systems of slightly earlier types Sc-Sd, for example M33, have masses of the order of $3 \times 10^{10} \mathfrak{M}_{\odot}$ with $\mathfrak{M} / \mathfrak{L} \simeq 5$ (Gordon, 1971; Huchtmeier, 1973; Warner et al., 1973).

Freeman (1970) has pointed out that if $\mathfrak{M} / \mathfrak{L}=$ constant in the disk, the exponential distribution of luminosity (Section 3 ) implies that the surface-density distribution of mass is also exponential $\varrho(r)=\varrho_{0} \exp (-\alpha r)$ and the total mass of the disk is 


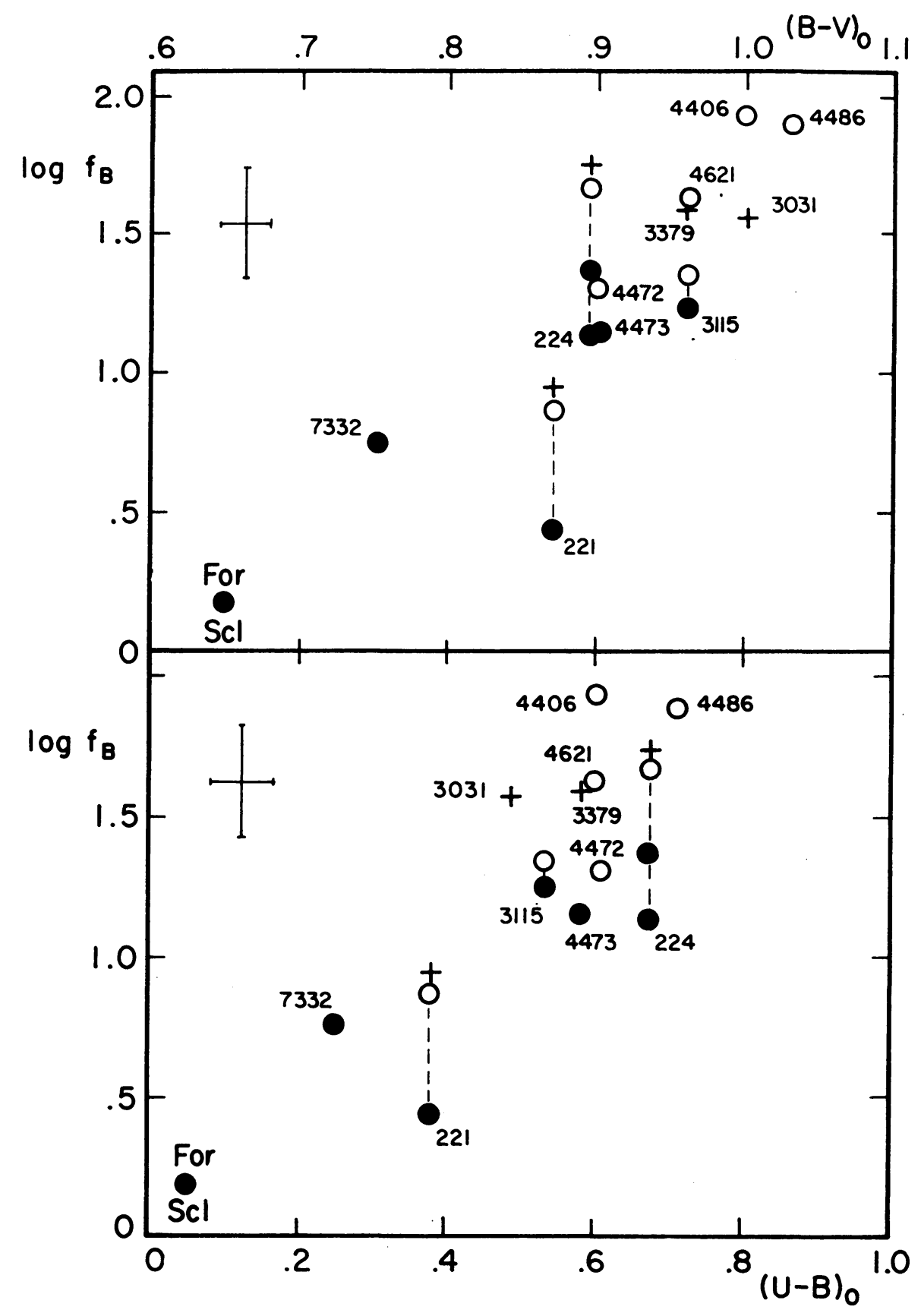

Fig. 10. Correlation between mass/luminosity ratio $f_{\mathrm{B}}$ and colour indices $(B-V)_{0},(U-B)_{0}$ for spheroidal systems, after Einasto and Kaasik (1973). Recent photoelectric estimates of $\sigma_{r}(0)$ tend to give lower masses (dots) than earlier photographic data (circles). Estimates of $f_{\mathrm{B}}$ from spectrophotometric models of galaxy populations (crosses) are also too high. 


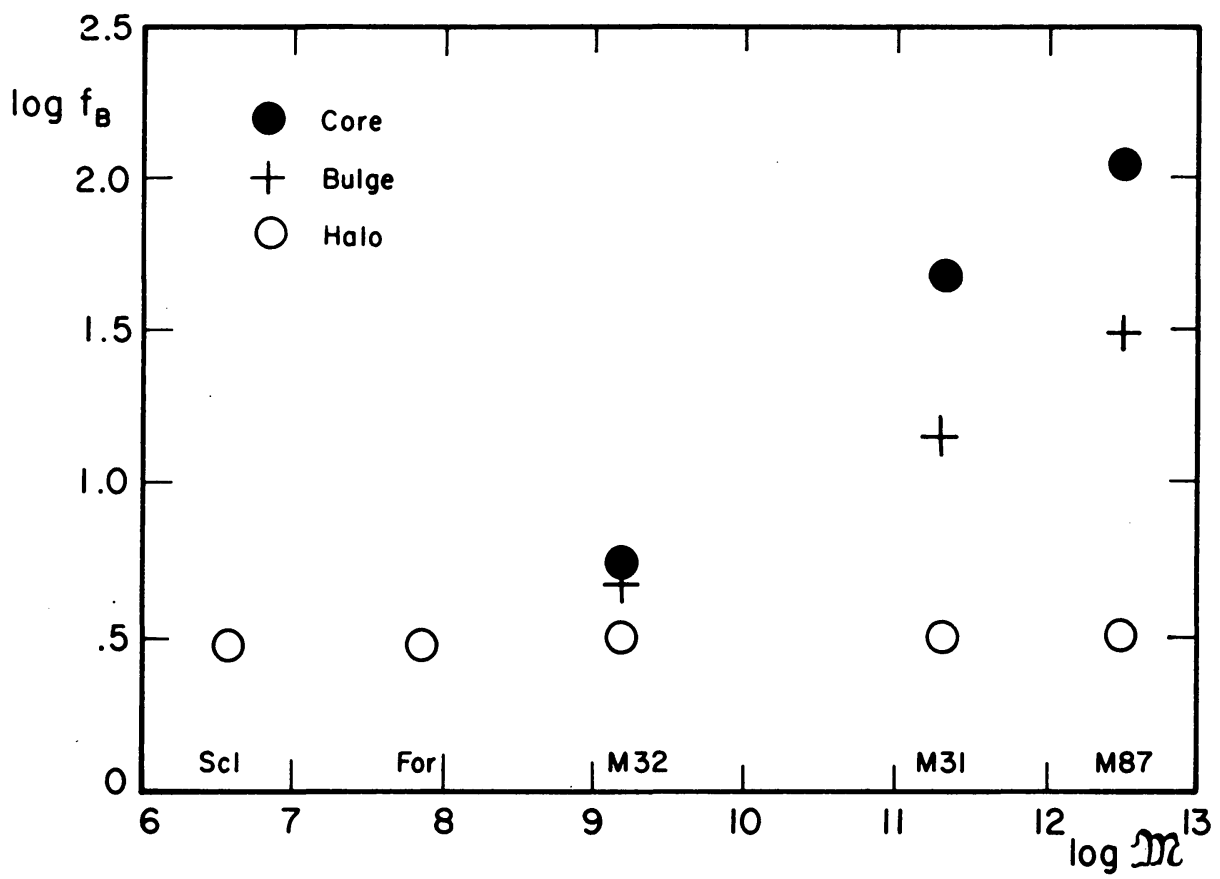

Fig. 11. Mass/luminosity ratio $f_{\mathrm{B}}$ in the core, bulge, and halo of spheroidal systems as a function of total mass $\mathfrak{M}_{\mathfrak{T}}$, after Einasto (1972). The halo component is the only population present in lowdensity dwarf ellipticals (Scl, For).

$\mathfrak{M}_{\mathrm{D}}=2 \pi \varrho_{0} / \alpha^{2}$. The calculated rotation curve of an exponential disk agrees well with observations of some flat systems.

\subsection{MiXED SYSTEMS: INDICATIVE MASSES}

In galaxies of intermediate types from $\mathrm{L}$ to $\mathrm{Sb}$ or $\mathrm{Sbc}$, both spheroidal and flat components contribute significantly to the total mass and potential energy of the system. Random motions and rotation make comparable contributions to the total kinetic energy and both must be taken into account (Oort, 1940, 1965). Realistic mass models of such galaxies are hampered by insufficient data on the velocity dispersion, and relatively detailed results are available only for a few systems including NGC 3115 (Oort, 1940), NGC 4111 (van Houten, 1961) and especially. M31 (Einasto, 1972b).

For statistical purposes the best that can be done at present is to compute an indicative mass $\mathfrak{M}_{\mathrm{i}}=V_{\mathrm{M}} R_{\mathrm{M}}^{2} / G \mu$, where the maximum rotational velocity $V_{\mathrm{M}}=V\left(R_{\mathrm{M}}\right)$ is derived from (a) optical and radio rotation curves for the larger galaxies, and (b) the total width $W$ of the $21-\mathrm{cm}$ profile (corrected for band-width and projection factors) for unresolved objects.

In case (b) $R_{\mathrm{M}}$ is not directly observable, but it may be estimated from an optical diameter with which it is correlated by case (a) data. Then the scale factor $\mu$ is chosen empirically so that on the average $\overline{\mathfrak{M}}_{\mathrm{i}} \simeq \overline{\mathfrak{M}}_{\mathrm{T}}$ for the galaxies with total masses derived by more detailed model fitting. 
For example, the Nançay group (Bottinelli et al., 1968; Heidmann, 1969) has adopted

$$
\mathfrak{M}_{\mathrm{i}}=3 \times 10^{4} a W^{2} \quad \text { (solar units), }
$$

where the isophotal diameter $a$ (in kpc) is on Holmberg's system (1958) and $W$ is the corrected total line width (in $\mathrm{km} \mathrm{s}^{-1}$ ). The coefficient gives $\left\langle\mathfrak{M}_{\mathrm{T}} / \mathfrak{M}_{\mathrm{i}}\right\rangle \simeq 1$ within a factor 3 for galaxies which have been best observed at $21 \mathrm{~cm}$. This precision is good enough compared with the mass range of hydrogen-rich galaxies which covers almost 3 orders of magnitudes.

An important result of this approach is a striking confirmation of the conclusion that $\mathfrak{M} / \mathfrak{L}=$ constant, independent of galaxy type for $t>0 \ddagger$ (Figure 15).

\section{Rotation Periods, Maximum Velocities, Angular Velocities and Angular Momenta}

Early analyses of line inclinations in galaxy spectra (Mayall, 1948, 1960; Mayall and Lindblad, 1970) revealed a loose correlation between rotation period $P$ and Hubble type, in the sense that early types rotate faster than later types; a range from $P<10^{7} \mathrm{yr}$ for $t<0$ to $P>10^{8}$ yr for $t>5$ was indicated. The correlation was rather loose because the angular velocity $\omega=2 \pi / P$ decreases rapidly from the centre outwards in most galaxies and the radius $R_{l}$ of the 'linear' inner region where $\omega \simeq$ constant varies with galaxy type (Figure 12) and also with apparent diameter (i.e. angular resolution). Nevertheless, the basic trend has been confirmed by more recent studies (e.g. Vorontsov-Velyaminov, 1970c; Table VI) and may be restated as a correlation between maximum (linear) rotational velocity $V_{M}$ and stage $t$ along the Hubble sequence (Brosche 1971). $\ddagger$

For spirals a definite correlation $(\varrho \simeq-0.8)$ (Figure 13) is present and in the mean

$$
V_{\mathrm{M}}=290-24 t \quad(2 \leqslant t \leqslant 10) \text {. }
$$

For $t<2$ the velocity dispersion $\sigma_{\mathrm{v}}$ becomes comparable to $\omega r$ and a distinction must be made between circular $V_{\mathrm{c}}$ and rotational $V_{\mathrm{r}}$ velocities (Oort, 1940, 1965; van Houten, 1961), but available data, especially on $\sigma_{v}$, are insufficient to determine whether the linear relationship continues for earlier types, including lenticulars.

\section{TABLE VI}

Mean rotation periods of galactic disks ${ }^{a}$

\begin{tabular}{llllll}
\hline Type & $\mathrm{L}, \mathrm{SO} / \mathrm{a}$ & $\mathrm{Sa}, \mathrm{ab}$ & $\mathrm{Sb}, \mathrm{bc}$ & $\mathrm{Sc}, \mathrm{cd}$ & $\mathrm{Sd}, \mathrm{m}$ \\
$\langle\log P\rangle$ & 7.77 & 7.80 & 8.16 & 8.29 & 8.45 \\
$n$ & 3 & 11 & 25 & 30 & 30
\end{tabular}

a in years for linear branch of velocity curve, after Vorontsov-Velyaminov (1970).

\# Evidence for this remarkable conclusion first appeared in 1968-69 (Ables, 1971; de Vaucouleurs et al., 1969a, b; Roberts, 1969; Vorontsov-Velyaminov, 1970b); it is in sharp contrast with ideas prevailing only 10 yr ago when the meagre data available suggested parallel decreases of $\mathfrak{M} / \mathfrak{L}$ and colour index as a function of stage along the Hubble sequence (cf. de Vaucouleurs, 1959b; Holmberg, 1964). $\ddagger$ A search has also been made for possible correlations between $\omega$ and $R_{1}$, or between $R_{1}$ and spectral indices for a given type Sb or Sc (Saslaw, 1970, 1971 a, b, 1972) but no significant relationship has been found. 


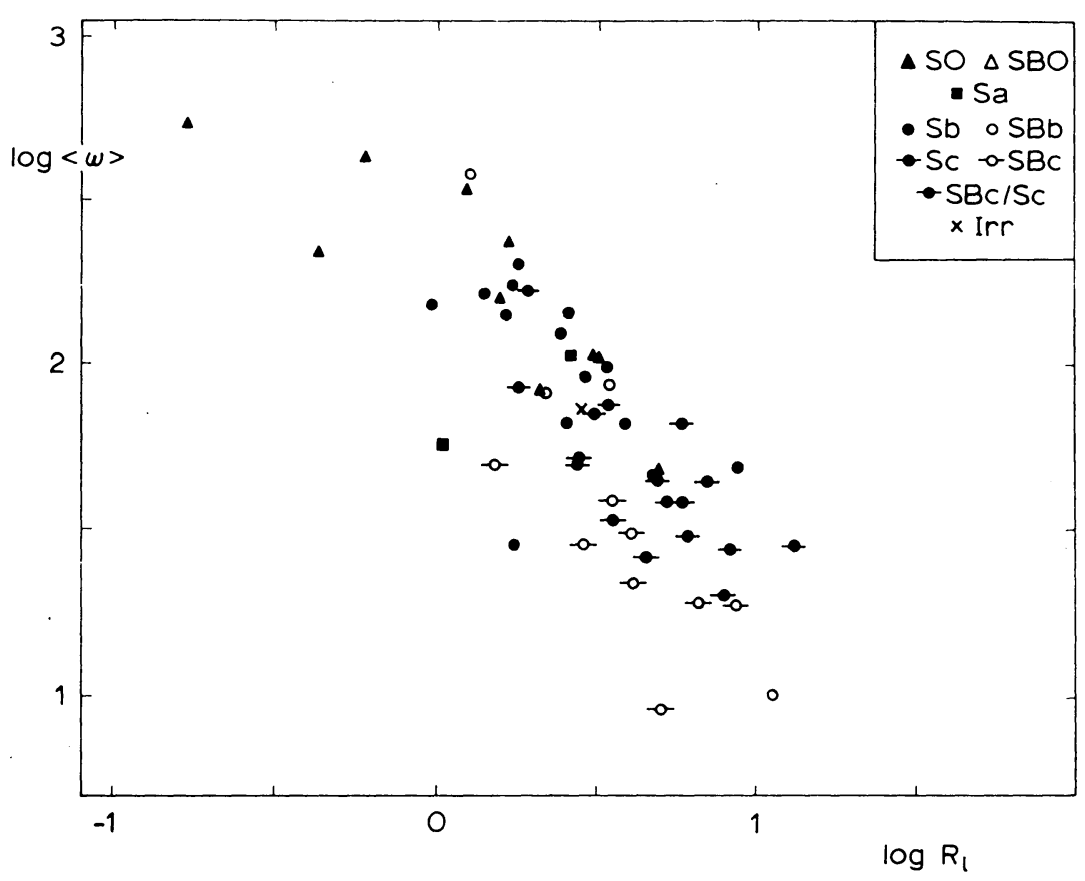

Fig. 12. Correlation between mean angular velocity $\langle\omega\rangle\left(\mathrm{km} \mathrm{s}^{-1} \mathrm{kpc}^{-1}\right)$ and radius $R_{l}(\mathrm{kpc})$ of linear part of rotation curve, after Mayall and Lindblad (1970).

There is also a correlation between $R_{\mathrm{M}}$ and other scale factors, such as the isophotal diameters in the BGC or Holmberg systems. In Equation(8) a mean value $\left\langle 2 R_{\mathrm{M}} \mid a\right\rangle=0.2$ was assumed, independent of type. There is good evidence, however, that this ratio is not a constant but varies with stage $t$ along the Hubble sequence as shown in Figure 14 and suggesting a possible improvement in estimates of indicative masses.

Angular momenta of galaxies (total $A_{\mathrm{T}}$ or per unit mass $A^{*}$ ) have attracted much interest in recent years (Brosche, 1963; Ozernoy, 1967; Takase, 1967; Takase and Kinoshita, 1967; Heidmann, N., 1969; Freeman, 1970) because it may be supposed that both mass and angular momentum are conserved as a primordial cloud contracts into a galaxy (Mestel, 1963).

For the larger galaxies with detailed velocity data, a mass distribution model $\mathfrak{M}(r)=2 \pi \int_{0}^{r} \mu(r) r \mathrm{~d} r$, derived from the rotation curve $V(r)$, is combined with it to estimate the angular momentum distribution

$$
A(r)=\int_{0}^{r} r V(r) \mathrm{d} \mathfrak{M}(r)=2 \pi \int_{0}^{r} r^{2} V(r) \mu(r) \mathrm{d} r
$$

and the total angular momentum $A_{\mathrm{T}}=A(\infty)$ ( $\mu=$ projected surface density).

For small galaxies, especially when $V_{M}$ only is given by the $21-\mathrm{cm}$ line width, an indicative specific angular momentum (per unit mass)

$$
A_{\mathrm{i}}^{*}=0.10 a V_{\mathrm{M}}
$$




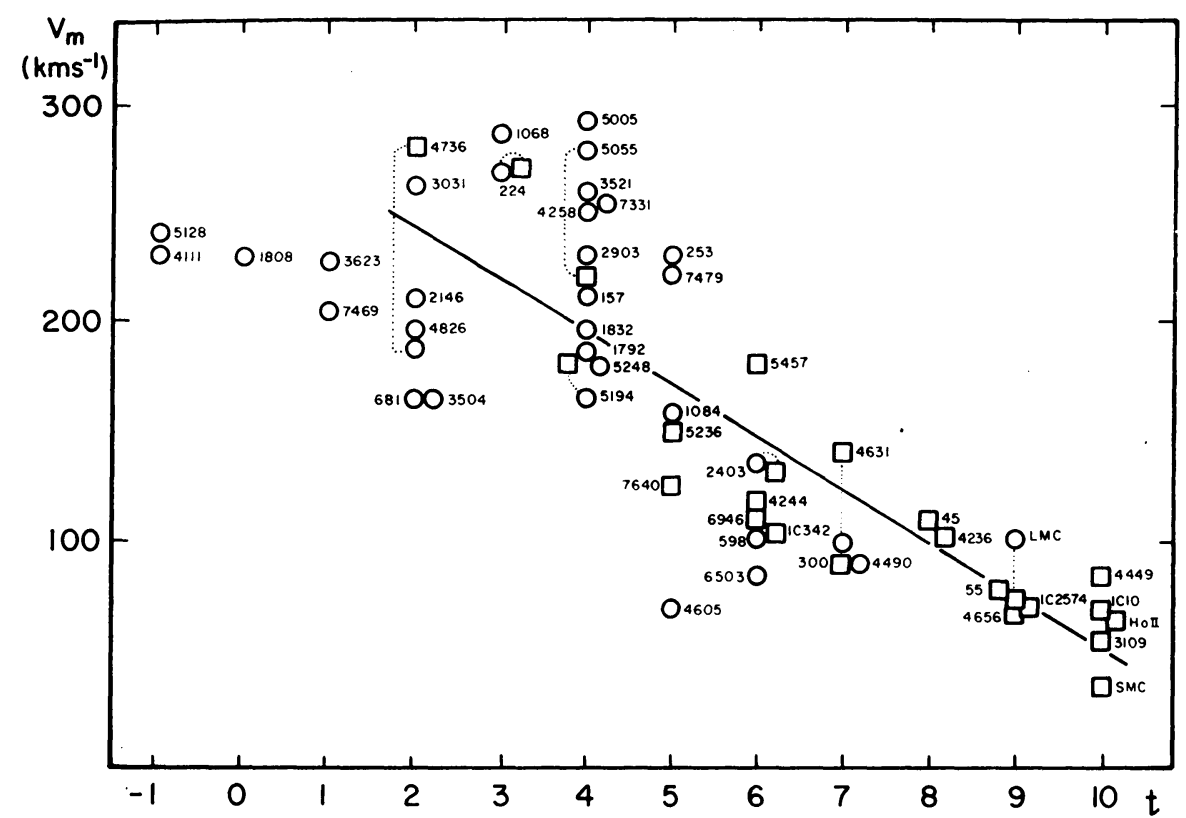

Fig. 13. Correlation between maximum rotation velocity $V_{M}$ and revised Hubble type, after Brosche (1971).

may be calculated (Heidmann, N., 1969). The numerical coefficient is chosen so that $A_{i}$ would be the actual angular momentum of a spinning solid disk of diameter $a / 5$.

Remarkably tight linear correlations between $\log A$ and $\log \mathfrak{M}$ have been found in the empirical data, i.e. $A \propto \mathfrak{M}_{\mathrm{T}}^{\theta}$; for example $A_{\mathrm{T}} \propto \mathfrak{M}_{\mathrm{T}}^{2}$ (Brosche, 1963) or, more precisely, $A_{\mathrm{T}} \propto \mathfrak{M}_{\mathrm{T}}^{7 / 4}$ (Takase and Kinoshita, 1967) or, again, $\mathfrak{M}_{i} \propto A_{\mathrm{i}}^{* 3 / 2}$ (Heidmann, N., 1969). However, Freeman (1970) has pointed out that for an exponential disk in centrifugal equilibrium

$$
A_{\mathrm{D}}=1.109\left(G \mathfrak{M}_{\mathrm{D}}^{3} \Lambda\right)^{1 / 2}
$$

and since $\mathfrak{M}_{\mathrm{D}}=2 \pi \mu_{0} \Lambda^{2}$ (Section 7.3) it follows necessarily that $A_{\mathrm{D}} \propto \mathfrak{M}_{\mathrm{D}}^{7 / 4}$. Freeman shows, further, that if $\mu_{0}=$ constant and $\mathfrak{M}_{\mathrm{D}}$ depends only on the scale parameter $\Lambda$, a relation of the form $\log A=\theta \log \mathfrak{M}_{\mathrm{D}}+C$, with $\theta \simeq 7 / 4$, will always obtain when $A$ and $\mathfrak{M}$ are derived from a two-parameter representation of the rotation curve (e.g. by a Brandt function), because of a fortuitous compensation of numerical coefficients. Conversely, if $A_{\mathrm{T}} \propto \mathfrak{M}_{\mathrm{T}}^{7 / 4}$ is established in an early phase of evolution of the protogalaxy (and if $A$ and $\mathfrak{M}_{\mathrm{T}}$ are conserved in the subsequent collapse to a disk), the constancy of $\mu_{0}$ would be a consequence.

\section{Interstellar Gas Content}

A small, but significant fraction of the mass of galaxies is in the interstellar gas, mainly neutral and ionized hydrogen, with a small admixture $(\sim 10 \%)$ of helium, heavy ele- 

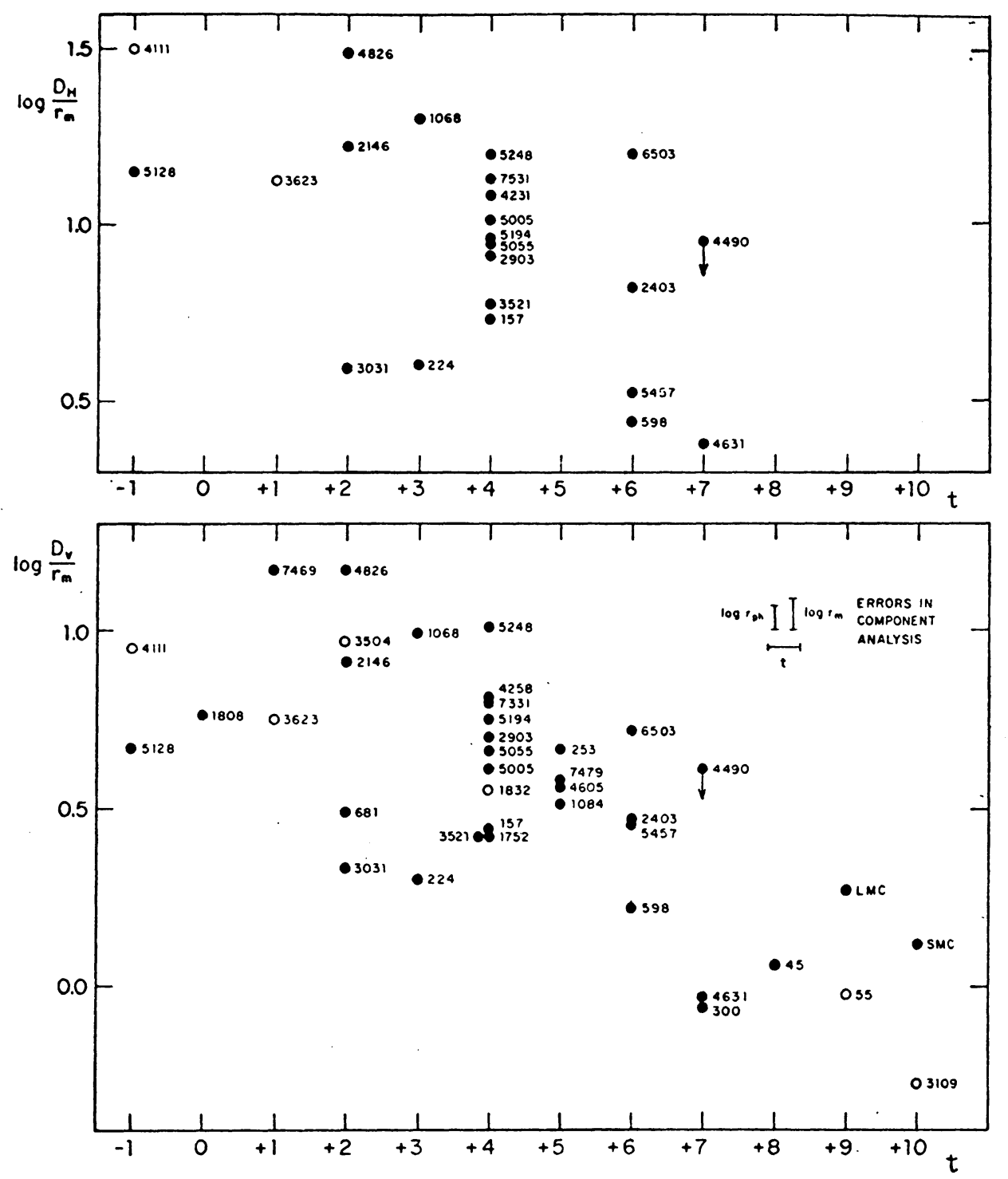

Fig. 14. Correlation between ratio of optical diameter $D$ to radius $R_{M}\left(V_{M}\right)$ at maximum rotation velocity with stage $t$ along revised Hubble sequence, after Brosche (1973). $D_{\mathrm{H}}$ is in Holmberg's system, $D_{\mathrm{v}}$ in BGC system. The ratio is clearly not constant, indicating that the numerical coefficient in Equation (8) should be a function of $t$.

ments and molecules. Whether this gas is a primordial remnant or a by-product of stellar evolution its presence is a dominant factor in the present make-up of galaxies. Although a variable and in some cases significant fraction of this gas is ionized, it is believed that with allowance for self-absorption (Heidmann et al., 1971) its mass can be estimated with little error by observation of the $21-\mathrm{cm}$ emission line. 


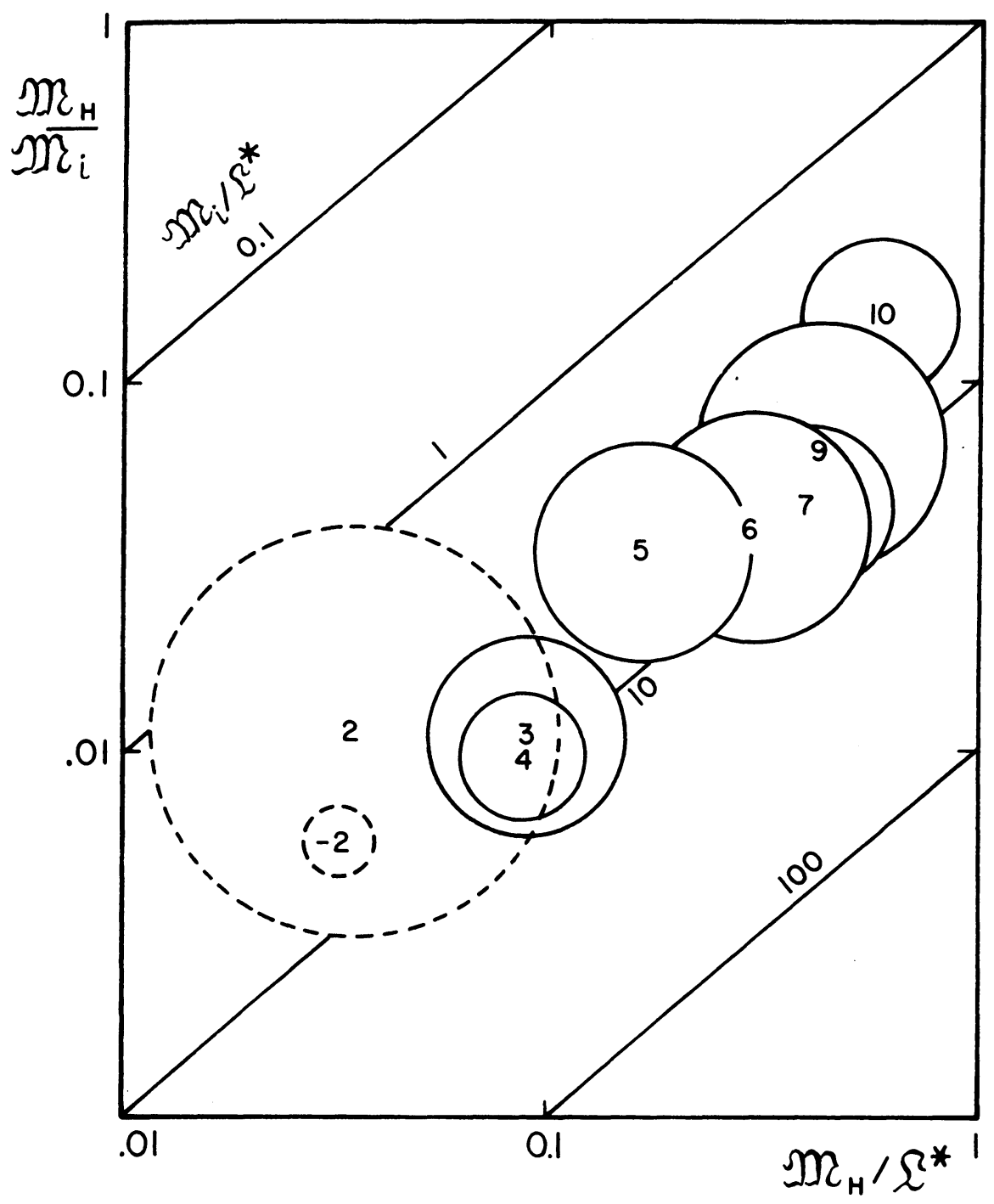

Fig. 15. Mass-luminosity ratio as a function of Hubble type and neutral hydrogen content, after N. Heidmann (1969). $\mathfrak{M}_{\mathrm{i}} / \mathfrak{L}^{*}$ is constant and independent of stage $t$ along spiral sequence $(t>0)$.

Some general conclusions may be drawn from the mass of data collected during the past decade, in particular with the large reflectors at Nançay, Parkes and Green Bank: ${ }^{\ddagger}$

(1) $\mathrm{H}$ I emission has now been measured for nearly 200 galaxies, mainly spirals and irregulars $(t \geqslant 2)$; very few lenticulars and early spirals are detectable. The total hydrogen mass $\mathfrak{M}_{\mathrm{H}}$ is strongly correlated with Hubble type $t$ and absolute luminosity $\mathfrak{Q}^{*}$ or indicative mass $\mathfrak{M}_{i}$.

¥ For reviews of results prior to $1969-70$ see Roberts $(1969,1972)$. 
For example Figure 16, after N. Heidmann (1969), displays the relationships between the three ratios of hydrogen mass $\mathfrak{M}_{\mathrm{H}}$, indicative mass $\mathfrak{M}_{i}$ and absorption-free blue luminosity $\mathfrak{Q}^{*}$ taken 2 by 2 as a function of Hubble stage $t$; we see that $\mathfrak{M}_{\mathrm{H}} / \mathfrak{M}_{\mathrm{i}}$ and $\mathfrak{M}_{\mathrm{H}} / \mathfrak{L}^{*}$ increase by nearly 2 orders of magnitude from early spirals to Magellanic irregulars, while $\mathfrak{M}_{\mathrm{i}} / \mathfrak{Q}^{*}$ remains nearly constant. In particular, the hydrogen-to-total mass ratio increases from $<1 \%$ in $\mathrm{L}$ types $(t<0)$ to $\sim 10 \%$ in $\mathrm{Sm}-\mathrm{Im}$ types $(t=10) . \mathfrak{M}_{\mathrm{i}}$ and $\mathfrak{M}_{\mathrm{H}}$ are plotted vs. $\mathfrak{L}^{*}$ and $t$ in Figure $16 \mathrm{a}, \mathrm{b}$; the largest total masses $\left(\mathfrak{M}_{\mathrm{T}}>3 \times 10^{11} \mathfrak{M}_{\odot}\right)$ are found among Sa to Sc supergiants, while the largest $\mathrm{H}_{\mathrm{I}}$ masses $\left(>10^{10}, \mathfrak{M}_{\odot}\right)$ occur in high-luminosity Sc and Sd galaxies (Balkowski, 1972).

(2) The density distributions of interstellar hydrogen in galaxies are known in some detail for only a few nearby objects having large apparent diameters and types $t \geqslant 3$. This small sample strongly suggests that the distribution of $21-\mathrm{cm}$ emission is closely correlated with that of luminosity in the flat (exponential) component, but with a lower density gradient. For example, near the centre of M31 where the spheroidal component is dominant, $\mathrm{H}$ I emission is weak or absent (Roberts, 1966) so that the $\mathrm{H}$ I distribution appears as a flat annulus more or less coinciding with the spiral structure in the disk; a similar situation was first noted in our Galaxy (Oort, 1965). In Magellanic spirals the $\mathrm{H}$ I surface density follows closely that of luminosity and star density, in particular that of the extreme population I component (de Vaucouleurs 1955, 1957b). More precisely, the effective diameters of the optical and $21-\mathrm{cm}$ distributions are very nearly equal ( $6^{\circ}$ in LMC, 12' in IC 1613; de Vaucouleurs and Freeman, 1972). Some minor departures from this general correlation are significant, especially an absence of concentration of $\mathrm{H}_{\mathrm{I}}$ in the bar of the Large Cloud where an older population component is dominant (de Vaucouleurs 1956b; McGee, 1964; McGee and Milton, 1966a, b; Walker et al., 1969)*.

In intermediate systems Sc-Sd, such as M33, the $\mathrm{H}$ i distribution is also intermediate. It could be represented by a "large double ring structure overlaid on a uniform disk with a central density depression of $35 \%$ " (Huchtmeier, 1973) and it is much less concentrated as well as more extensive than the optical distribution.

(3) Smaller galaxies observed at lower relative resolution (Bottinelli, 1970, 1971) provide additional information. In particular, statistical comparisons of ring vs. gaussian models for 33 galaxies suggest that the ring model may be appropriate in only $1 / 3$ of the cases. The $\mathrm{H}$ I diameter, defined as the half-intensity width $\dot{a}_{\mathrm{H}}$, is loosely correlated with the isophotal diameter in the Holmberg system $(\varrho \simeq 0.5)$ and Hubble type, with

$$
\left\langle\log a_{\mathrm{H}} / a\right\rangle=-0.45+0.046 t \quad(t \geqslant 3)
$$

\footnotetext{
* Walker et al. refer to the bar population on their IR photographs of the Large Cloud as 'Population II'; this identification is questionable because neither the stellar luminosity function (de Vaucouleurs, 1956b), nor the integrated spectrum (de Vaucouleurs and de Vaucouleurs, 1959a, b) match that of a globular cluster or of a Sculptor type dwarf elliptical (Population II by definition), but are appropriate for an old (or ageing) Population I. This population was labelled type Ic to differentiate it from the 'zero age' type Ia and possible intermediate age type Ib (de Vaucouleurs, 1956b; Figure 2). Star counts in the core of the Small Cloud (Schilt et al., 1955) lead to similar conclusions.
} 


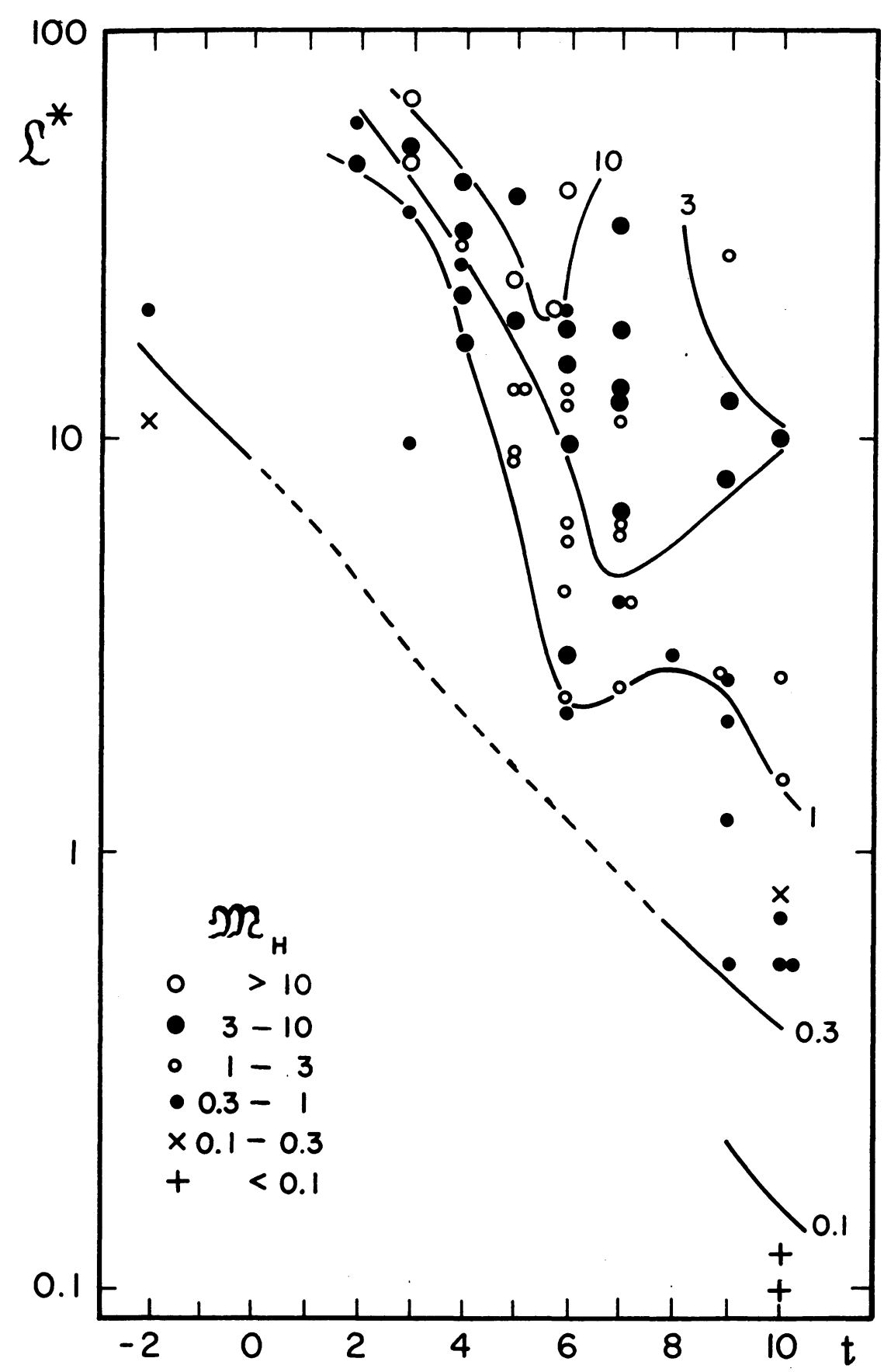

Fig. 16a.

Fig. 16. Correlations between hydrogen mass $\mathfrak{M}_{\mathrm{H}}$, indicative mass $\mathfrak{M}_{\mathfrak{i}}$, absolute luminosity $\mathfrak{L}^{*}$ and revised type $t$, after $\mathrm{N}$. Heidmann (1969). (a) $\mathfrak{M}_{\mathrm{H}}$ (in $10^{9} \mathfrak{M}_{\odot}$ ) as a function of $\mathfrak{L}^{*}$ and $t$. (b) $\mathfrak{M}_{\mathrm{i}}$ (in $10^{9} \mathfrak{M}_{\odot}$ ) as a function of $\mathfrak{L}^{*}$ and $t$. 


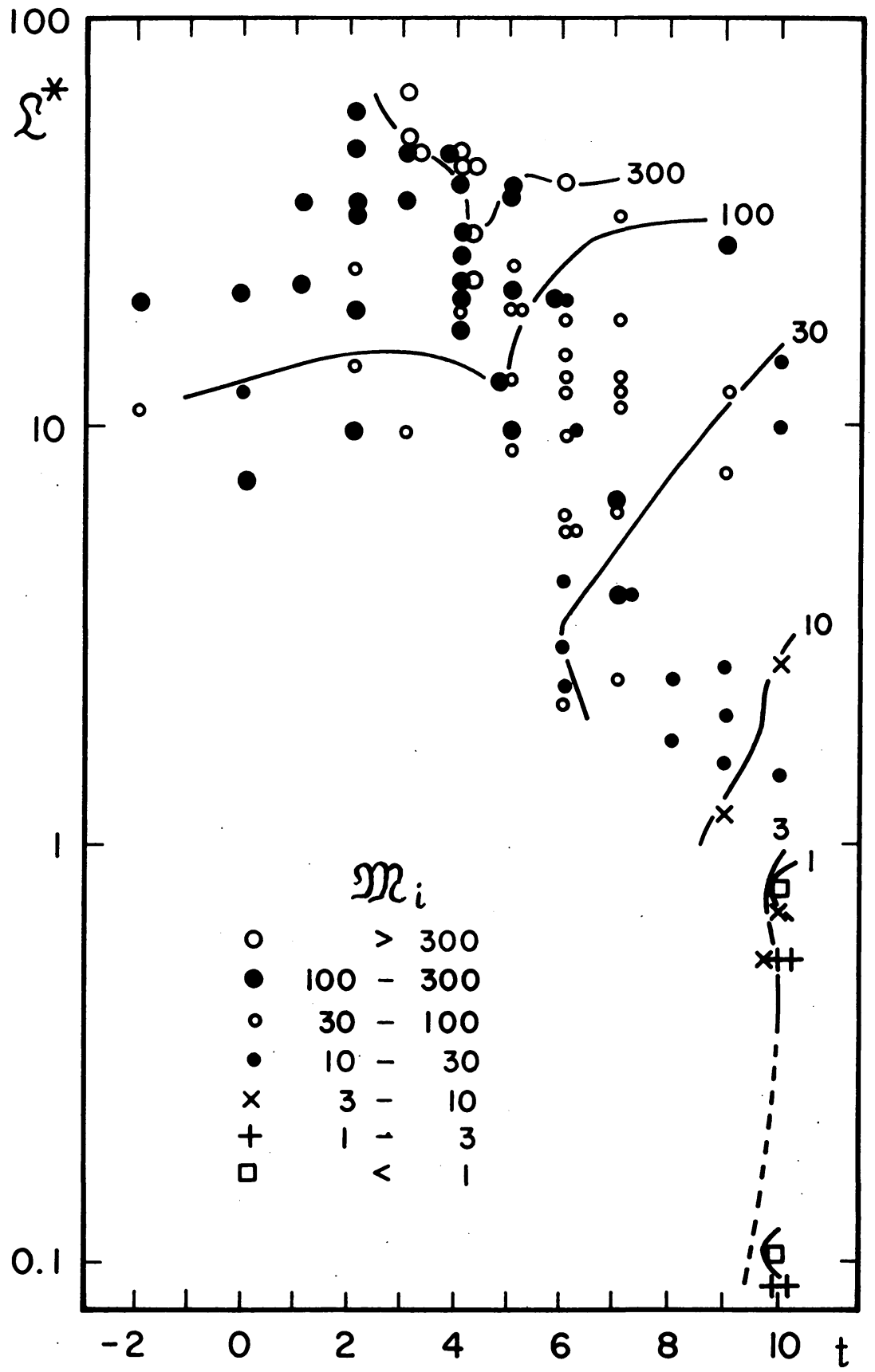

Fig. 16b. 


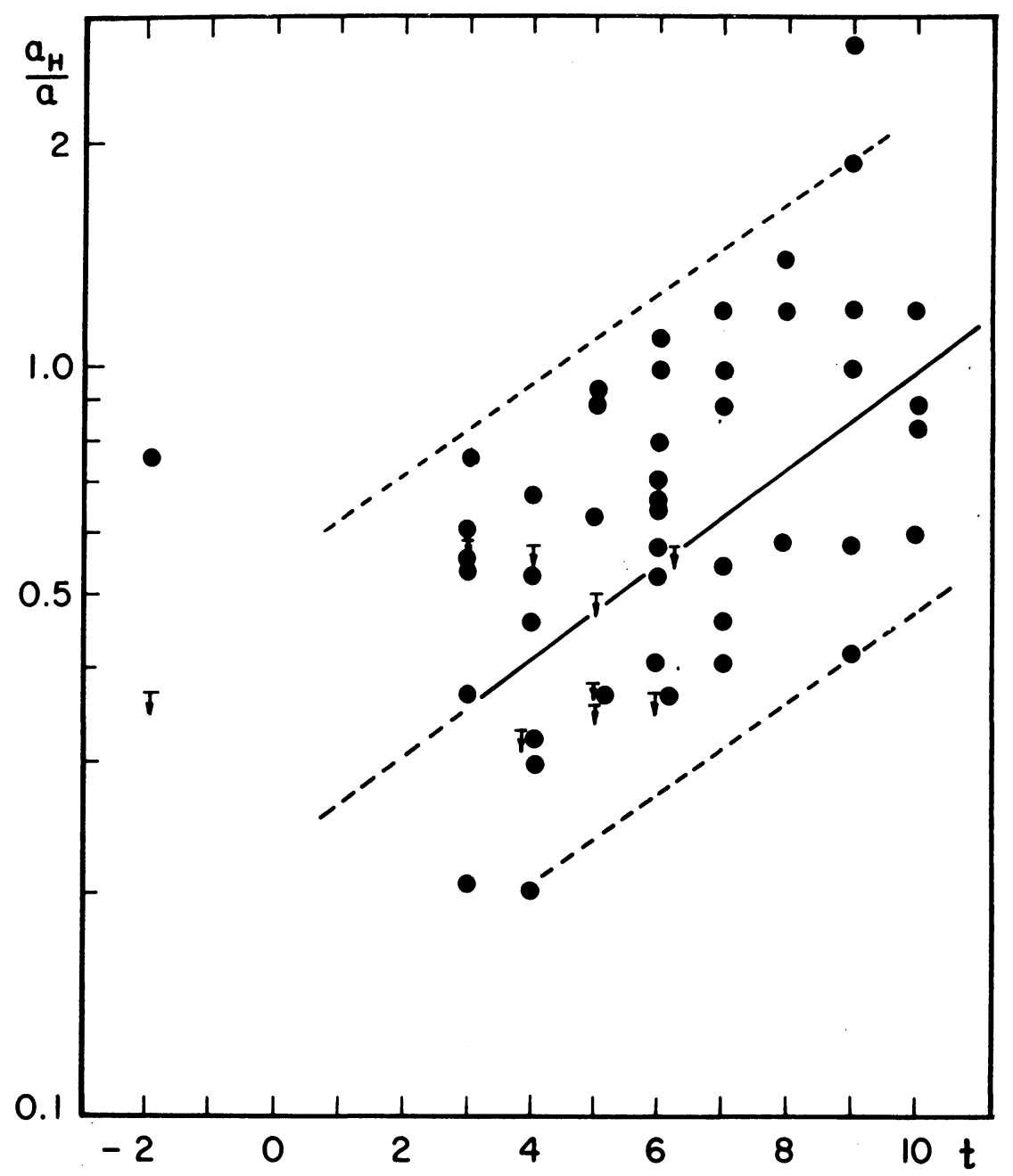

Fig. 17. Correlation between ratio of hydrogen to isophotal diameters $a_{\mathrm{H}} / a$ and revised type $t$, after Bottinelli (1970). $a_{\mathrm{H}}$ is the half--intensity $\mathrm{H}$ I diameter, $a$ is isophotal diameter in Holmberg's system.

thus $a_{\mathrm{H}} \simeq \mathrm{a}$ in the Magellanic irregulars $(t=10)$, decreasing to $a_{\mathrm{H}} \simeq a / 2$ in the early spirals (Figure 17). It follows that the relation between mean hydrogen surface density $\sigma_{\mathrm{H}}^{*}$ and type depends on how the diameter is defined; using optical diameters it was initially concluded that $\sigma_{\mathrm{H}}^{*}$ increases toward later types (Gouguenheim, 1969), a result that was regarded as consistent with the parallel increase in the rate of star formation, abundance of extreme Population I supergiants, etc. However, when $\mathbf{H}$ I diameters are used, $\sigma_{\mathrm{H}}^{*}$ appears to be very nearly independent of morphological type, with an average $\left\langle\sigma_{\mathrm{H}}^{*}\right\rangle \simeq 1 \mathrm{mg} \mathrm{cm}^{-2}$ for $t>0$ (Figure 18).

(4) Recently, special efforts were made to detect $21-\mathrm{cm}$ emission from early type galaxies $(t<0)$; weak emission could be detected from lenticulars $(-3<t<-1)$ 


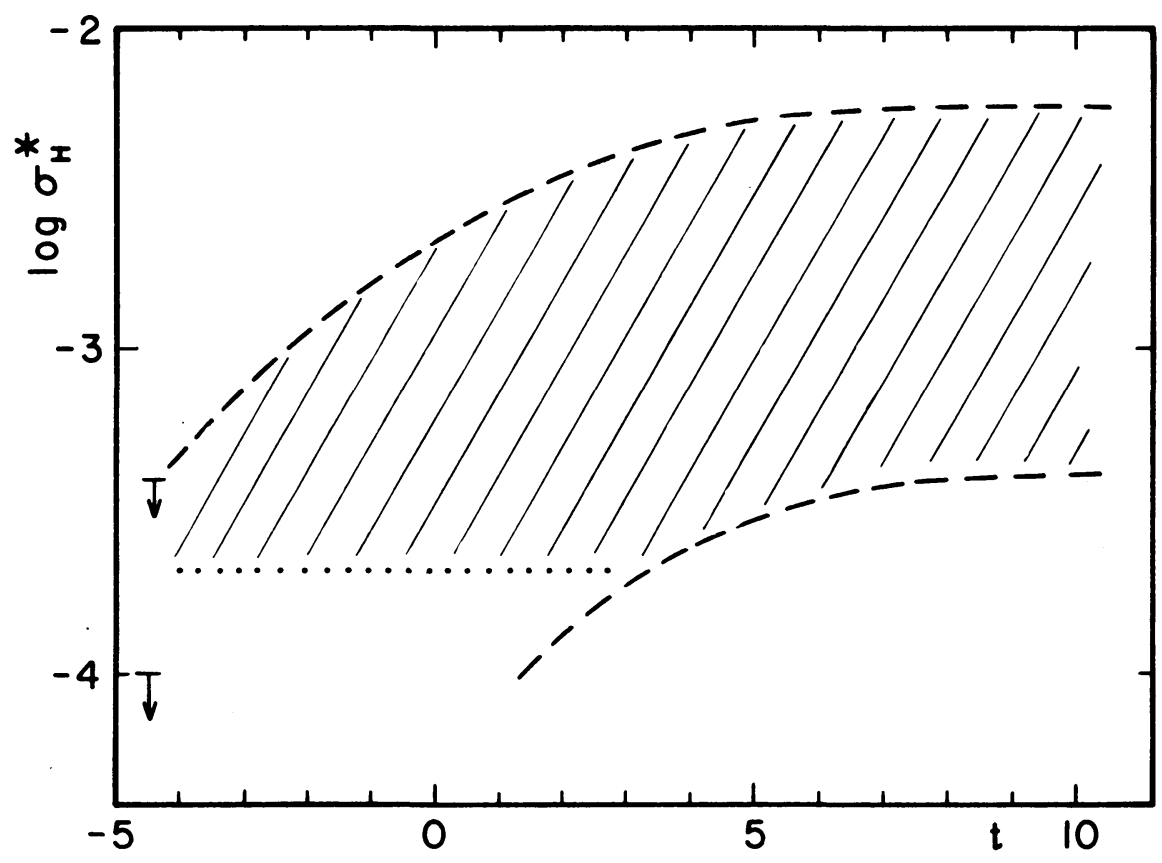

Fig. 18. Average surface density of neutral hydrogen $\sigma^{*}{ }_{\mathrm{H}}\left(\mathrm{g} \mathrm{cm}^{-2}\right)$ as a function of revised type $t$, adapted from Balkowski (1972). The average density $\sigma^{*} \mathrm{H} \simeq 1 \mathrm{mg} \mathrm{cm}^{-2}$ is nearly independent of $t>0$ when calculated for half-intensity diameter $a_{\mathrm{H}}$ of $\mathrm{H} \mathrm{I}$ distribution.

(Balkowski et al., 1972), but none from normal ellipticals (Gallagher, 1972; Bottinelli et al., 1973; Kerr, 1973). The upper limits for the latter appear to be much lower than could have been expected from an extrapolation to $t<-3$ of the trend of $\mathfrak{M}_{\mathrm{H}} / \mathfrak{M}_{\mathrm{i}}$ and $\sigma_{\mathrm{H}}^{*}$ vs type (Figure 19); this result suggests that a discontinuity in physical properties exists between $\mathrm{E}$ and $\mathrm{L}$ galaxies. A similar conclusion was reached independently from analyses of optical properties (luminosity distribution laws, ellipticities) (Sections 3,4).

Optical line emission from interstellar gas in the centres of $\sim 10$ to $20 \%$ of the elliptical galaxies has been known for a long time (Mayall, 1939), sometimes with a remarkably high velocity dispersion $\left(\sigma_{\mathrm{v}} \simeq 500-1000 \mathrm{~km} \mathrm{~s}^{-1}\right)$; the best known examples are NGC 4278 and 4486 (Minkowski and Osterbrock, 1959; Osterbrock, 1960). This gas is believed to come from evolving stars and may be abnormally rich in heavy elements because, if the ratio $(Y+Z) / X$ of helium + heavy elements to hydrogen has the 'normal' value $\sim 0.1$, there is an apparent conflict between current estimates of

(a) the rate of mass loss by corpuscular radiation from evolving stars, $\sim 1 \mathfrak{M}_{\odot} \mathrm{yr}^{-1}$ (Gallagher, 1972; van den Bergh, 1972b),

(b) the mass of interstellar gas responsible for the observed line emission, $\sim 10^{5} \mathfrak{M}_{\odot}$ in NGC 4278 (Osterbrock, 1960), and

(c) the upper limit to the mass of neutral hydrogen, which is probably less than $10^{8} \mathfrak{M}_{\odot}$ in NGC 4472 (Bottinelli et al., 1973; Kerr, 1973).

Taking the numbers at face value, (c) is $10^{-2}$ of what (a) would produce in $10^{10} \mathrm{yr}$; 


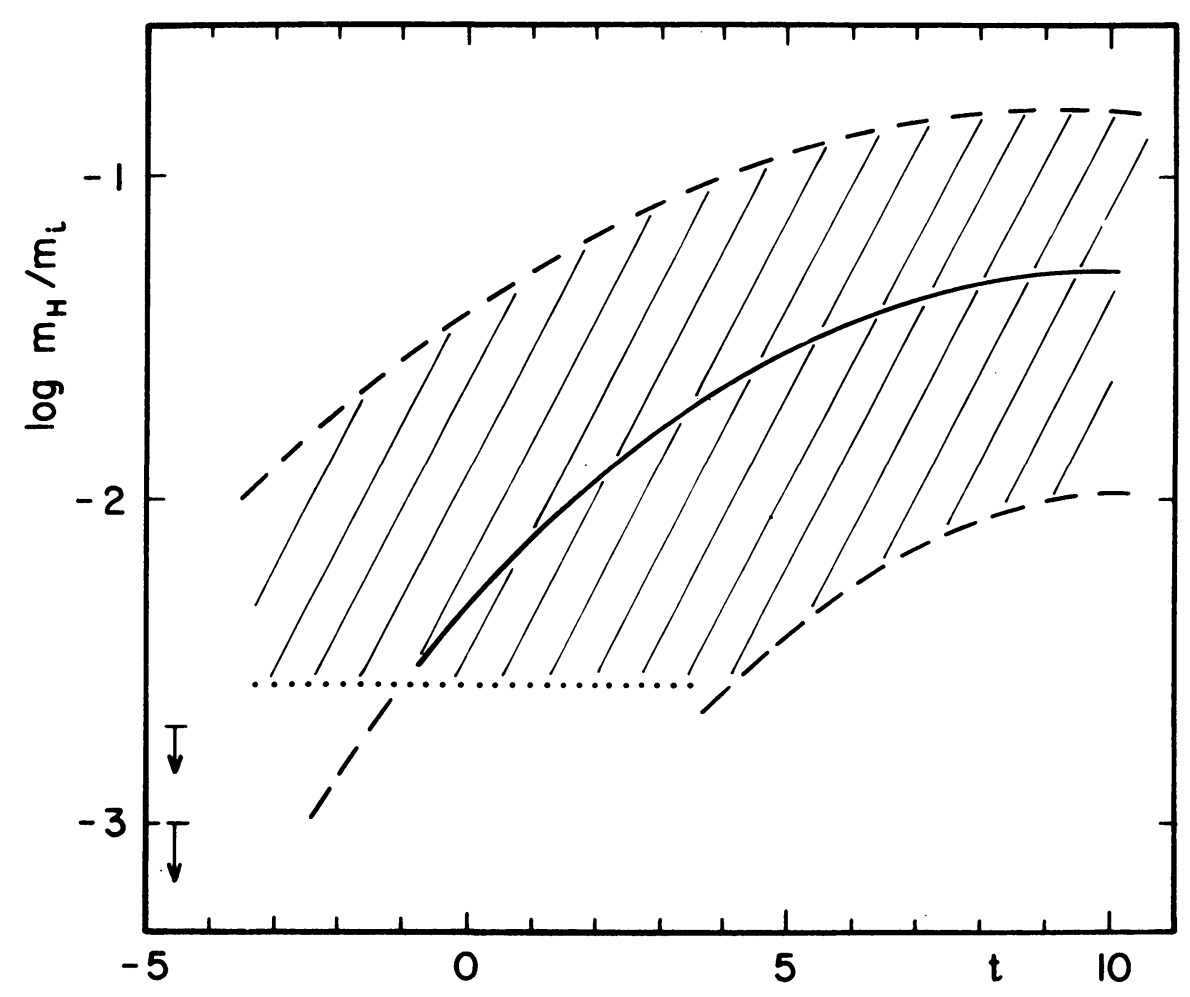

Fig. 19. Ratio of neutral hydrogen to indicative mass $\mathfrak{M}_{\mathrm{H}} / \mathfrak{M}_{\mathrm{i}}$ as a function of revised type $t$, adapted from Balkowski (1972).

this suggests that intermittent bursts of star formation at intervals $\sim 10^{8} \mathrm{yr}$ efficiently remove the accumulated gas. (A few ellipticals, e.g. NGC 185 and 205, are known to have blue stars near their centres). It is well to remember, however, that (a), (b) and (c) are all uncertain by \pm 1 order of magnitude, that a large fraction of the gas might be ionized, that the $(Y+Z) / X$ ratio may be higher in the centres of $E$ galaxies than in the disks of spirals where its 'normal' value is defined (Roberts, 1972), that a fraction of the gas might be ejected (e.g. in supernova exposions) at velocities exceeding the escape velocity and, if $\varrho_{H}^{*}$ is low enough, may actually escape from the galaxy. In this case, also, the $21-\mathrm{cm}$ line profile might be so broad and the emitting region so large that the line could easily have escaped detection (Bottinelli et al., 1973), and if so then (c) would be greatly underestimated.

\section{Spectral Energy Distributions and Colour Indices}

Information on the integrated spectral energy distribution functions of galaxies of different types is still fragmentary. Because of the basic differences in the compositions of the spheroidal and flat components, spectral data on the nuclear region of a galaxy are not in general applicable to the rest of the galaxy. During the past few years reliable 
data became available on the integrated spectra of giant ellipticals (Whitford, 1971; Schild and Oke, 1971) which form a remarkably homogeneous group (Lasker, 1970; Sandage, 1972), but it was not until recently that similar information was secured for other major types spanning the whole Hubble sequence from E to Im (Wells, 1972; 1973) (Figure 20).

These studies at intermediate resolution $(\sim 50 \AA)$ provide a link between high resolution $(\sim 1-5 \AA)$ work on galactic nuclei for detailed population analyses from line intensities (de Vaucouleurs and de Vaucouleurs, 1959a, b; Spinrad et al., 1971), and medium resolution ( $\sim 100-200 \AA$ ) multicolour surveys (Wood, 1966; Tifft, 1961-1969;

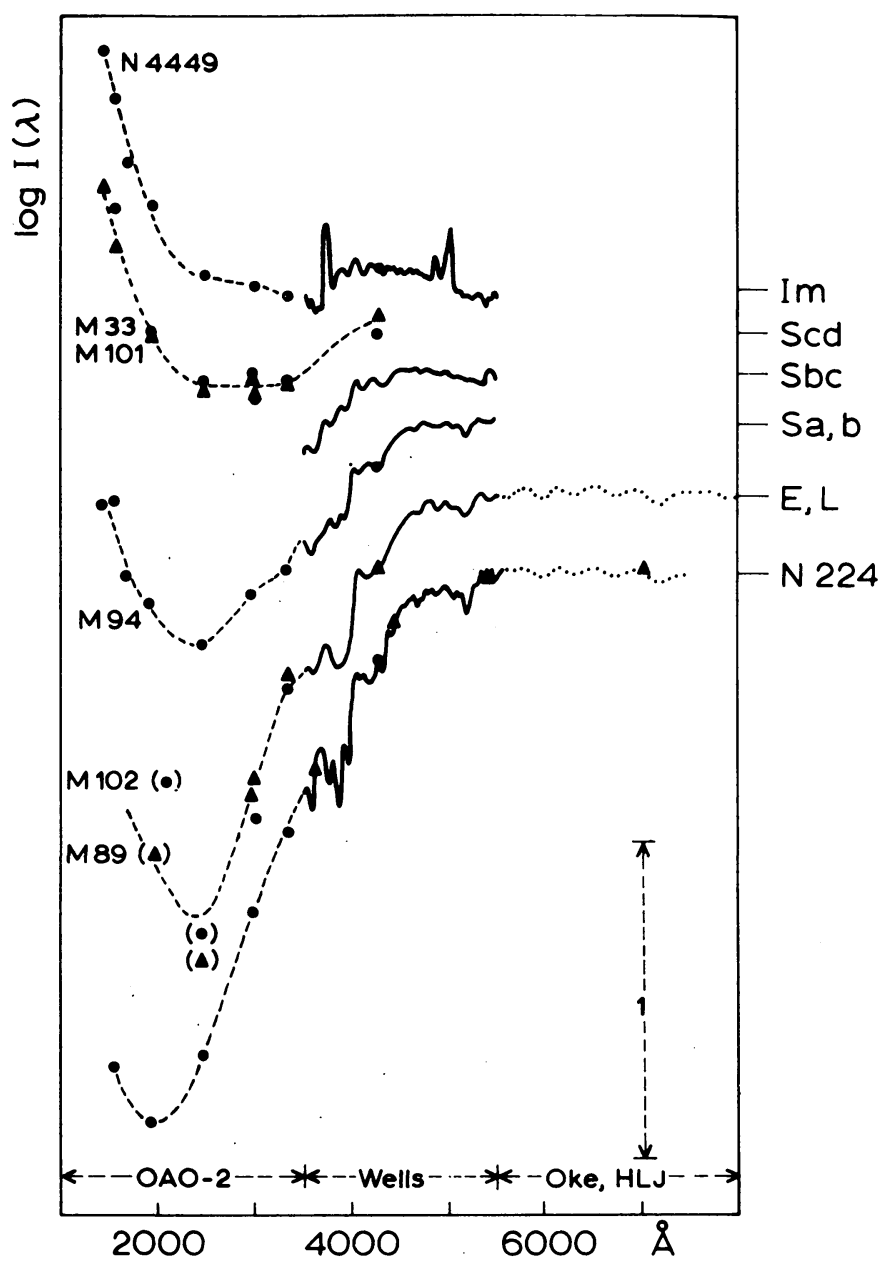

Fig. 20. Spectral energy distribution in galaxies of different morphological types along Hubble sequence from OAO-2 and ground-based data (identified at bottom). Galaxies in OAO set are identified at left and matched at $\lambda 4400 \AA$ to continuous curves from McDonald scanner observations (Wells, 1972) for corresponding galaxy types identified at right. Spectrum of N224 is for central region only. 
Westerlund and Wall, 1969). Fortunately, principal component analysis (Martin and Bingham, 1970) demonstrates that only 3 independent parameters, of which 2 are dominant, can be defined by a narrow-band 12-colour system (Wood, 1966). Therefore, a broad-band 3-colour system generating 2-colour indices $U-B, B-V$ already provides much spectral information. $U B V$ data are now available for over 1200 galaxies (de Vaucouleurs and de Vaucouleurs, 1964, 1972, where older references are given). New analyses of this material confirm and refine earlier conclusions:

(1) $U-B$ and $B-V$ colour indices, corrected for aperture, redshift, inclination and line emission effects, are closely correlated with each other (Figure 21) and with stage along the Hubble sequence (Figure 22). There is a smooth colour transition from reddish pure spheroidal systems $(t<-3)$ with intrinsic colours $U-B=+0.45$, $B-V=+0.90$ to blueish flat systems $(t>5)(-0.2,+0.45)$; in the range $-3<t<+6$ the relation is very nearly linear: $(U-V)_{0}=+0.98-0.12 t$.

(2) At a given stage $t$, galaxies in the different families $(A$ or $B)$ and varieties $(r$ or $s)$

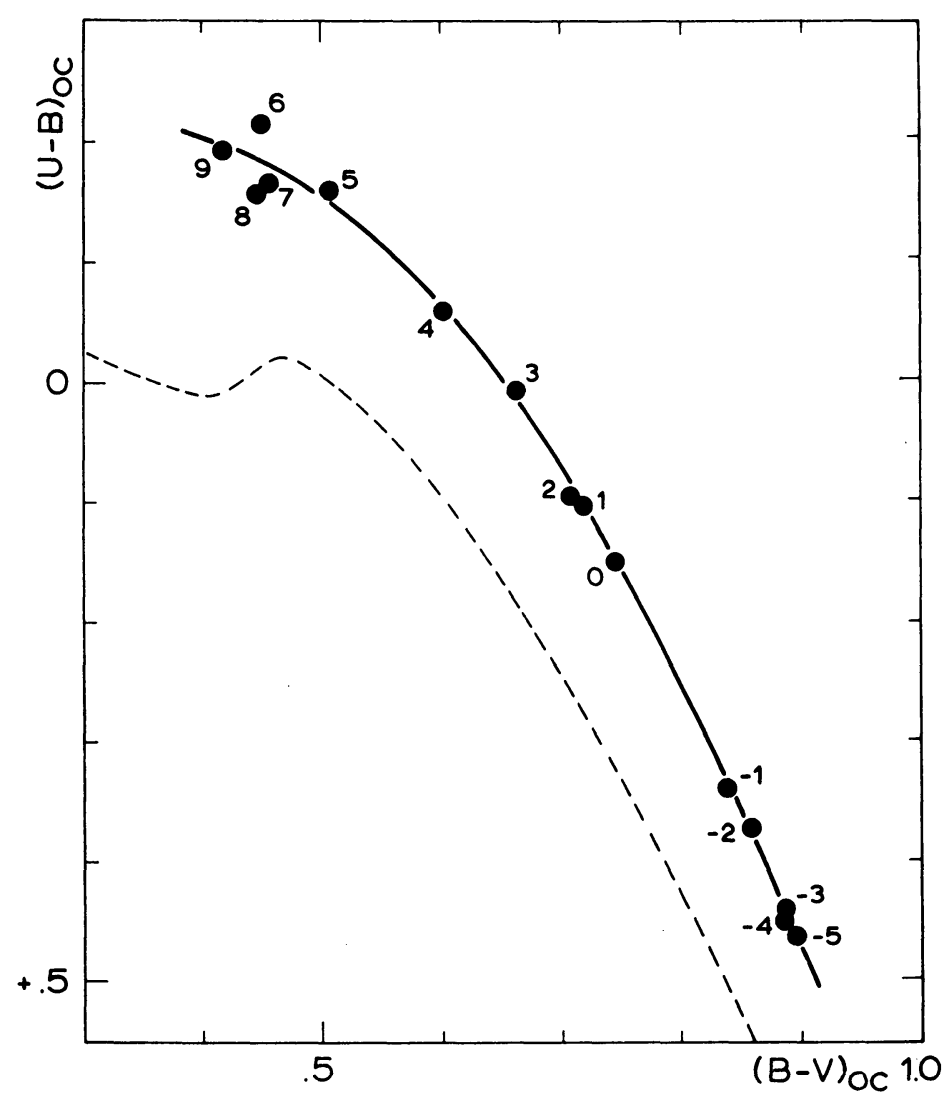

Fig. 21. Mean colour-colour relation for normal galaxies as a function of stage $t$ (G. \& A. de Vaucouleurs, 1972). Colours are corrected for galactic absorption, redshift and inclination effects.

Displacement from stellar main sequence (dashed) results from compositeness of galaxy spectra. 


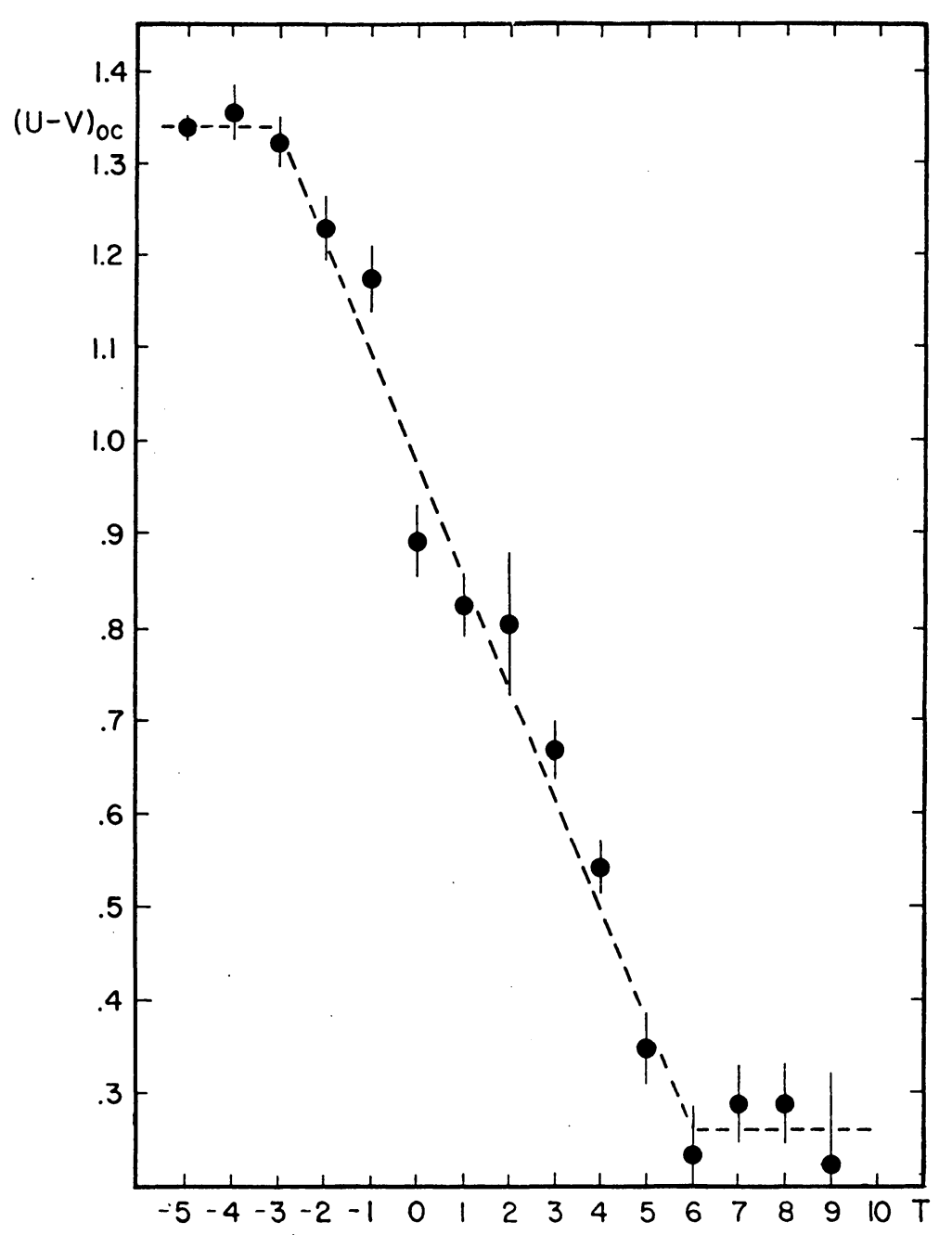

Fig. 22. Relation between mean corrected $U-V$ colour and stage $t$ (G. \& A. de Vaucouleurs, 1972). The relation is linear within the statistical errors in the range $-3 \leqslant t \leqslant 6$.

have the same colours; this suggests that structural details reflect relatively minor dynamical differences, not basic physical properties. Photometric and $21-\mathrm{cm}$ studies lead to the same conclusions (Sections 3 and 9).

(3) The absorption-free or metallicity index $Q_{0}=(B-V)_{0}-0.72(U-B)_{0}$ varies little with Hubble type for normal giant galaxies, increasing from 0.56 at $E$ to 0.65 at $\mathrm{Sb}(-5 \leqslant t \leqslant 3)$, and decreasing past $\mathrm{Sb}$ back to 0.55 at $\mathrm{Sm}(3 \leqslant t \leqslant 9)$. This is merely a way of saying that the colour-colour relation is very nearly linear and parallel to the reddening line.

(4) Normal galaxies of types earlier than $\mathrm{Sc}$ - and possibly of all types - are redder toward the centre, except for line emission effects. The radial colour gradient is very 
small in ellipticals and Magellanic irregulars (some of which are perhaps bluer in the centre), but large in early-type spirals where it is associated with strong radial gradients in various 'line strength' indices (McClure, 1969) and other spectral characteristics (McClure and van den Bergh, 1968) indicative of rapid changes in the stellar population mix or in the chemical composition, in particular with respect to metal abundances.

The presence of 'super-metal-rich' stars has been postulated to account for the strength of absorption lines from neutral metals and molecules ( $\mathrm{MgH}, \mathrm{TiO})$ in the nuclear region of M31 and other giant galaxies of early types (Spinrad et al., 1971).

(5) There is a significant correlation between integrated colours and absolute luminosities of elliptical and lenticular galaxies first noted 15 yr ago (Baum, 1959; de Vaucouleurs, 1961) and confirmed many times since (McClure and van den Bergh, 1968; Faber, 1971; Sandage, 1972; de Vaucouleurs and de Vaucouleurs 1972) (Figure 23). Low density dwarf ellipticals fainter than $M \simeq-15$ have the colour characteristics of metal-poor globular clusters $\left(Q_{0} \simeq-0.45\right)$; high density dwarfs, however, have normal or nearly normal colours (de Vaucouleurs, 1961; Faber, 1971).

(6) Ultraviolet multicolour photometry $(1200<\lambda<3300 \AA)$ with the OAO-2 satellite (Code et al., 1972) correlates well with $U B V$ data and follows similar colour-colour and colour-type relationships at wavelengths $\lambda>2400 \AA$. At shorter wavelengths an unexpectedly steep rise is observed in the spectral energy curve (per unit wavelength) of each galaxy type (Figure 20); it is even steeper than the $\lambda^{-4}$ function for a black body

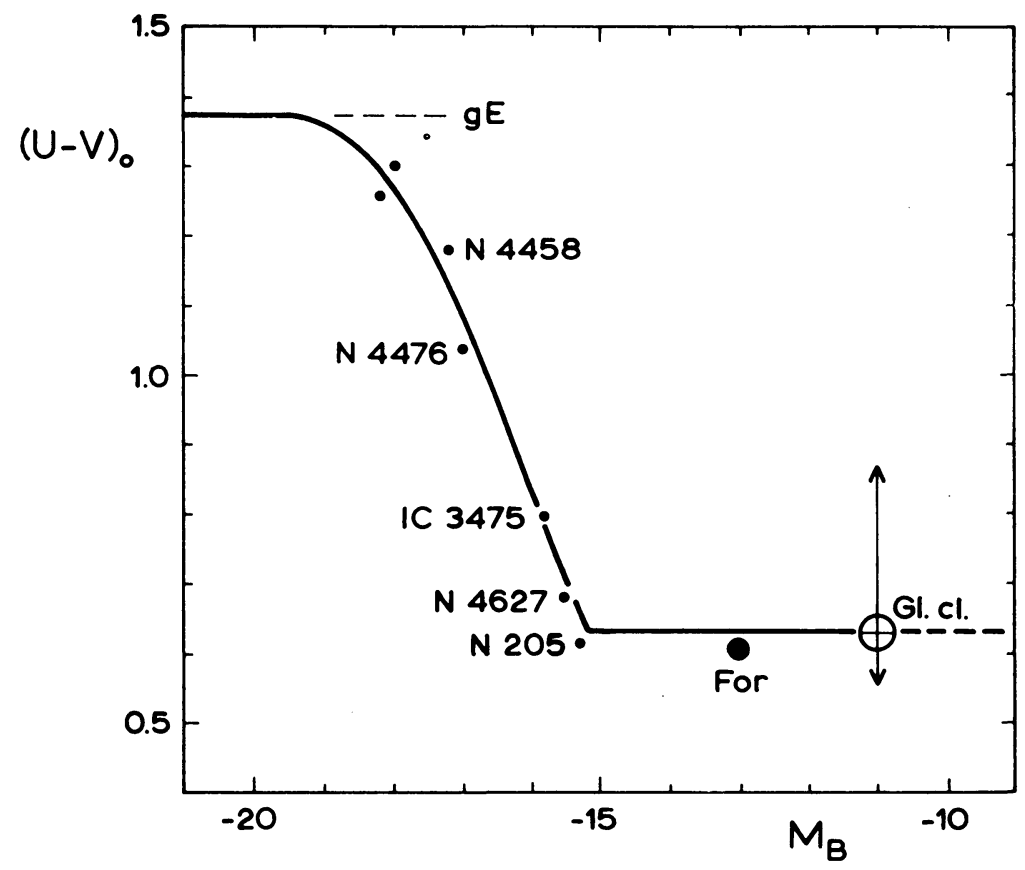

Fig. 23. Luminosity-colour relation for spheroidal systems (de Vaucouleurs and Ables, 1968). The intrinsic $U-V$ colour of elliptical and lenticular galaxies is closely correlated with absolute magnitude $M_{\mathrm{B}}<-15$. A few typical examples are illustrated. 
at infinite temperature. The resulting deep minimum of the energy curve near $\lambda=2400 \AA$ stands out most clearly in early type galaxies whose thermal continua are very weak in the UV as could be expected from the optical spectra, but it is still marked in the Magellanic irregulars whose UV spectra are dominated by the strong continua from their OB supergiants (Figure 20).

The favoured interpretation of the minimum invokes absorption and scattering by interstellar or possibly circumstellar grains (Gilra, 1971, 1972) with an absorption maximum near $\lambda \simeq 2200 \AA$ and strong forward scattering with high albedo at $\lambda<2200 \AA$. The implication of a significant dust component in E galaxies is interesting but, as the OAO experimenters point out, the detection of early type galaxies was marginal and needs confirmation*.

(7) Infra-red data are still very limited (Johnson 1966), except for a small and strongly biased sample of peculiar sources (Kleinmann and Low, 1970 a, b; Rieke and Low, 1972) in particular Seyfert galaxies, which have abnormally high IR fluxes, apparently correlated with radio continuum emission. Such galaxies emit $10^{22}-10^{24}$ W $\mathrm{Hz}^{-1}$ at $10 \mu$ compared with $10^{21}-10^{24} \mathrm{~W} \mathrm{~Hz}^{-1}$ at $21 \mathrm{~cm}$. The IR luminosity in the $7.9-13.3 \mu$ band is in the range $10^{35}$ to $10^{38} \mathrm{~W}$. Normal galaxies are much weaker IR emitters $\left(10^{32}\right.$ to $\left.10^{38} \mathrm{~W}\right)$ and in most or all cases the IR source is localized in the nucleus or the nuclear region.

\section{Independent Parameters}

A large number of measurable properties of galaxies $(m)$ are mutually correlated; the number of possible combinations of $m$ parameters 2 by 2 can be very large and the results confusing. It is important to discover: (1) how many (say, $p$ ) of these $m$ parameters are really independent, and, if possible, (2) which of those $p$ parameters have the most basic physical significance. The empirical correlations between observables could then be re-interpreted in terms of a small number of fundamental physical parameters such as mass, length and time scales, or energy and angular momentum, etc.

In the past few years the methods of principal component analysis (Deeming, 1968) have been applied to multi-colour photometry and other observables. An analysis of Wood's (1966) 12-colour photometry by Martin and Bingham (1970) suggested that only 2 or possibly 3 independent variables are sufficient to describe colour properties. From a more complete analysis of $n=31$ galaxies with respect to $m=6$ variables (type $t$, radius $R_{\mathrm{M}}$ and maximum velocity $V_{\mathrm{M}}$, face-on photometric radius $r_{\mathrm{p}}=a / 2$, luminosity $L$ and colour index $C$ ) Brosche (1973) concludes that just 2 independent variables contribute $82 \%$ of the total variance. The residual variance is mainly due to noise in the data (measuring errors, approximations in the face-on corrections, departures from linearity). Except for this noise, the original variables $x_{i j}$ (normalized to $\left.\sigma_{j}=1\right)$ are given by $x_{j}=\eta_{j 1} \xi_{1}+\eta_{j 2} \xi_{2}$, where $\xi_{1}, \xi_{2}$ are uncorrelated, rectangular

* Code et al. (1972) lump together M102 (NGC 5866) and M89 (NGC 4552) as examples of 'ellipticals'; it should be noted that M102 is a late lenticular $\left(\mathrm{SO}^{+}, t=-1\right)$ seen edge-on, with a well-known dust belt (see e.g. Sandage, 1961 ; Burbidge and Burbidge, 1960). 


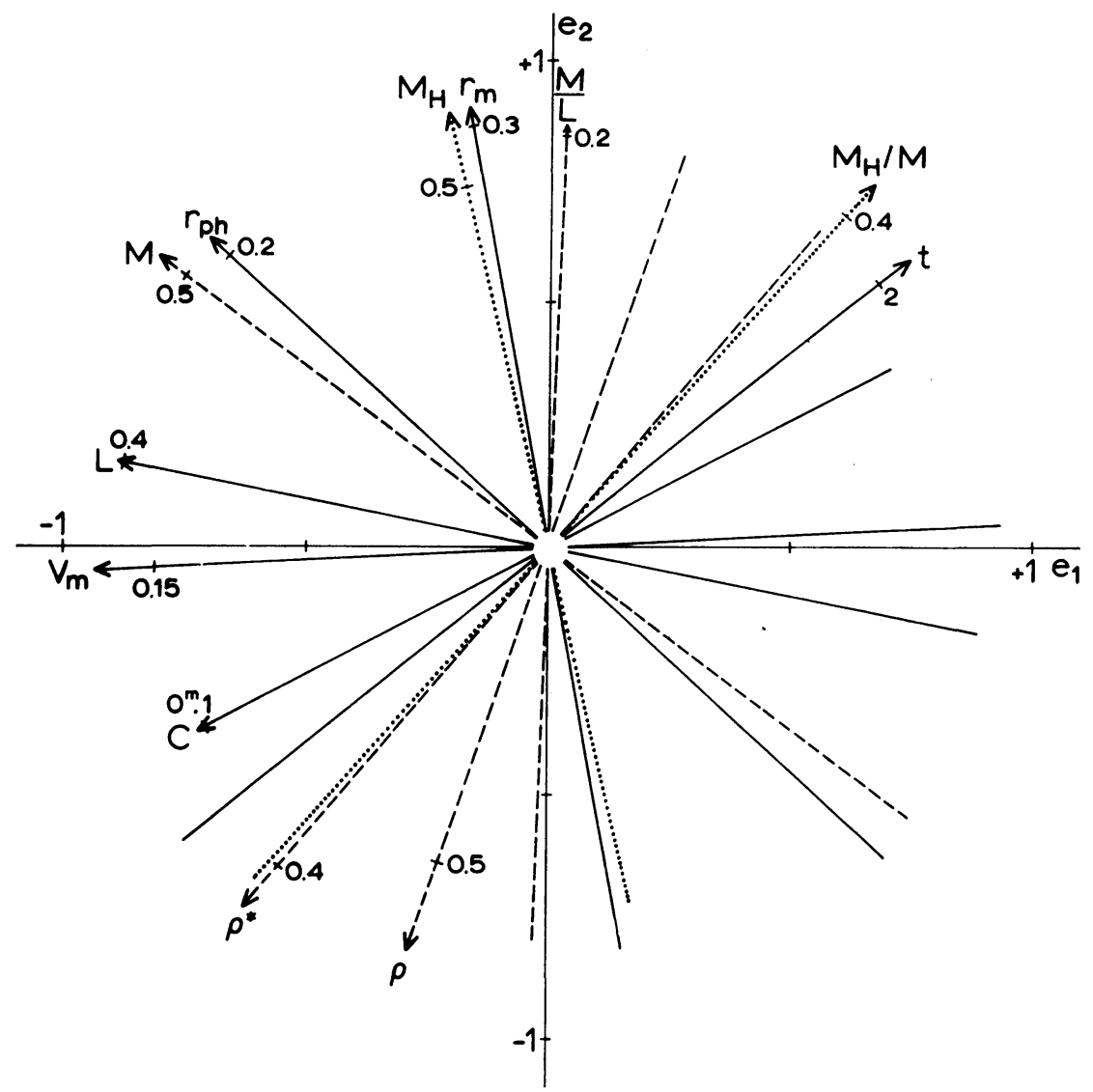

Fig. 24. Gradients of variables (observable and derived parameters $t, r_{\mathrm{m}}, V_{\mathrm{m}}, r_{\mathrm{p}}, \mathfrak{i}^{*}, C, M_{\mathrm{H}}, M, \varrho$ ) in eigenvector plane, after Brosche (1973). Gradients of uncorrelated variables are mutually rectangular.

coordinates. In this $\xi_{1} \xi_{2}$ plane the lines $x_{j}=$ constant are parallel to a vector $\mathbf{J}\left(\eta_{1 j}, \eta_{2 j}\right)$ with slope $\eta_{2 j} / \eta_{1 j}$ (Figure 24). Highly correlated variables are represented by $\mathbf{J}$ vectors forming a small angle, uncorrelated variables by orthogonal vectors. From the former, the (linearized) relations between correlated variables (or linear functions of same, e.g. space density $\varrho^{*}$, mass-luminosity ratio $f$, etc.) may be obtained; for example $\log f=0.009 \xi_{1}+0.233 \xi_{2}+0.257$. From the latter, the location of a galaxy in the manifold may be represented by its projection onto the $\xi_{1}, \xi_{2}$ plane (Figure 25).

Thus factor analysis tends to support the conclusions of the Nançay group (from a large number of 2- and 3-dimensional correlograms) that optical and 21-cm properties depend on two dominant and more or less independent parameters. The two most significant of the independent empirical parameters are the stage $t$ along the Hubble sequence and the absolute luminosity $\mathfrak{L}^{*}$, or some photometric radius $r_{\mathrm{p}}$ with which $\mathfrak{L}^{*}$ is strongly correlated (cf. Holmberg, 1969; Heidmann et al., 1971). The corresponding basic physical parameters may tentatively be identified with total mass and 


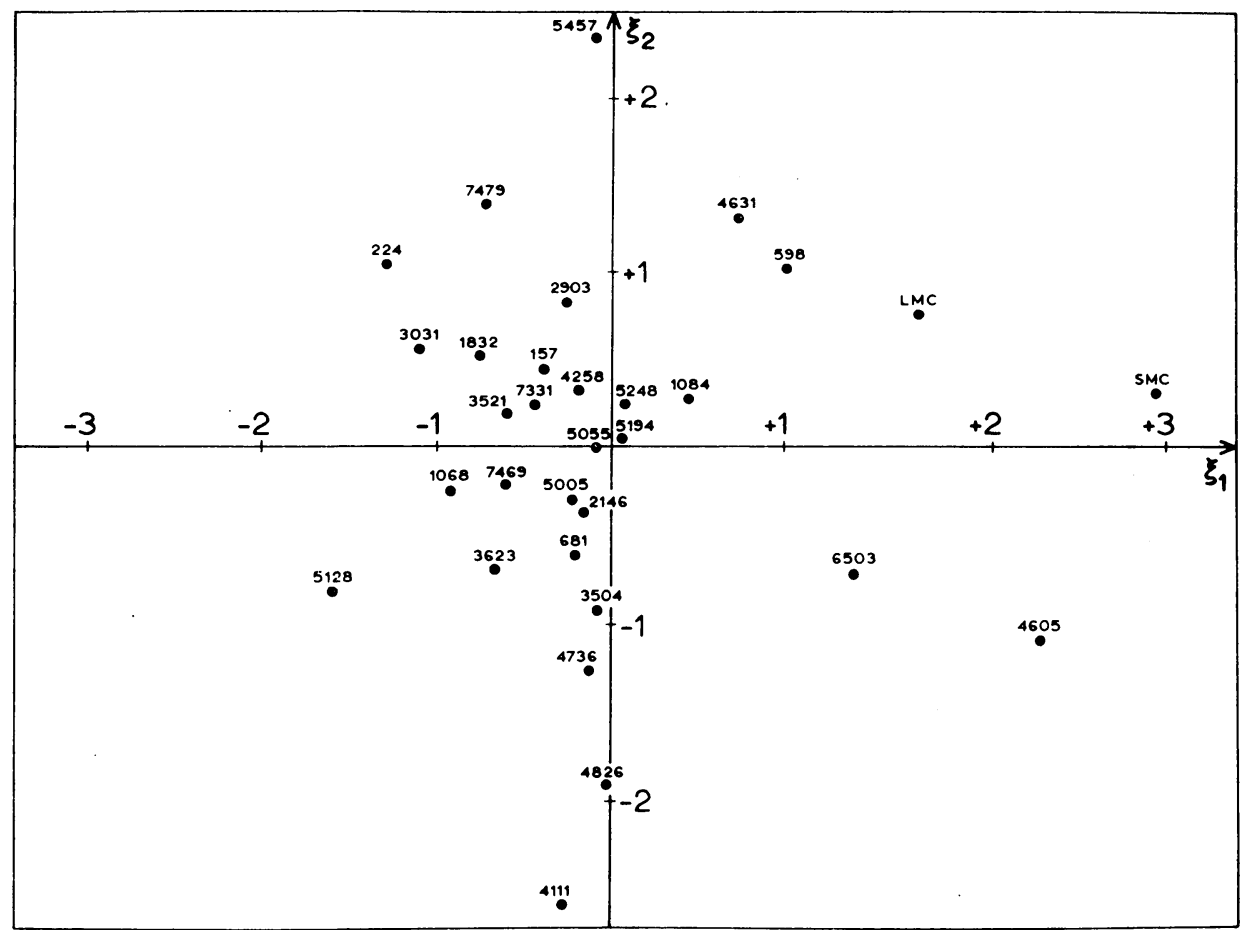

Fig. 25. Projections of galaxy representative points in $x_{j}$ space onto the eigenvector plane, after Brosche (1973). Stage $t$ increases from lower left to upper right; total masses increase from lower right to upper left, parallel to the gradient vectors of Figure 24.

total angular momentum (Brosche, 1971), although it is by no means proven that these two quantities suffice to determine all the others.

\section{Luminosity Functions}

Spectral data over a sufficiently large range of wavelengths are only beginning to appear and are still too few, especially in the infra-red, to allow meaningful estimates of the bolometric luminosity functions (LF) of normal galaxies. For the present, attempts to derive luminosity functions must still rely mainly on various catalogues and photographic magnitudes affected by ill-defined errors and selection effects. Until a few years ago most of the discussions were based on the subset of Shapley-Ames galaxies having radial velocities (from Humason et al., 1956) with corrections for incompleteness based on the questionable assumptions of spatial homogeneity and selection by apparent magnitude only (Kiang, 1961 ; van den Bergh, 1961). More recent studies of groups and clusters (Abell, 1962; Holmberg, 1969) using new magnitude data may be more directly applicable to unit volumes of space but, because of the small volumes and mode of selection of the data, the validity of the derived LF needs to be verified by independent analysis of residuals in the $(m, \log z)$ relation. 
Since we are not immediately concerned here with the absolute value of the average space density of matter or luminosity, the general agreement between the shapes of the functions derived by the different approaches is encouraging (Figure 26). It is interesting to remark that the general LF of galaxies turns out to be very nearly the sum of the gaussian and exponential functions originally proposed by Hubble (1936) and by Zwicky (1957).

More specifically the gaussian component with mean $\bar{M}_{\mathrm{pg}} \simeq-18.5$ and dispersion $\sigma_{M} \simeq 0.8 \mathrm{mag}$ (cf. de Vaucouleurs, 1958b) appears to describe mainly the giant spirals $(0 \leqslant t \leqslant 6)$, while the exponential component with slope 0.2 applies to earlier and later types. In other words, there are no dwarf spirals Sa to Sc, a fact first noticed by Shapley. However, the gaussian component includes also some giant ellipticals and lenticulars, at least in clusters (Figure 27). The corresponding integrated luminosity function $N\left(M_{\mathrm{i}}>M\right)$ can be roughly approximated in the observable range by two straight segments, as noted by Abell $(1962,1972)$.

\section{Clustering and Space Distribution}

Statistical studies of nearby groups and of the space distribution of galaxies (de Vaucouleurs, 1965, 1971) bring out a number of general properties of galaxies that may be relevant to the problem of their formation and evolution:

(1) The majority of galaxies are not isolated and randomly distributed in space but are members of small groups similar to the Local Group (Karachentsev, 1967; Zonn, 1968); less than 10 to $20 \%$ of the largest or nearest galaxies are not clearly members of identified groups (de Vaucouleurs, 1965; Corwin, 1967). Apparently isolated galaxies such as NGC 1313, 2903, 6744 and 6946 do not seem to differ in any of their properties from galaxies of the same type and luminosity in groups.

(2) The galaxy population of small, loose groups and clouds is dominated by spirals and Magellanic irregulars often to the point of an almost total absence of giant ellipticals and lenticulars. The Local Group and nearest groups illustrate this point (Figure 28). Examples of almost 'pure' clouds of spirals are the Grus Cloud (de Vaucouleurs, 1956a), the UMaI cloud (Morgan, 1958a) and the M94 group (van den Bergh, 1960).

(3) Dwarf ellipticals and irregulars are strongly concentrated in the same areas as spirals and appear as satellites of the giant spirals and not as an independent, dominant population of space (Reeves, 1956; van den Bergh, 1959; Vorontsov-Velyaminov and Noskova, 1971; Karachentseva, 1972).

It follows that the faint branch of the luminosity function (Figures 26, 27) derived from counts in groups or clusters may tend to overestimate the abundance of dwarfs in space. It is very difficult at present to make allowance for this bias.

According to Holmberg (1969) the space distribution of these companions with respect to their primaries is anisotropic, with a deficiency near the plane, and an excess near the minor axis of the primaries (Figure 29). Ejection from the nucleus of the primary has been suggested as a possible explanation of this strange 


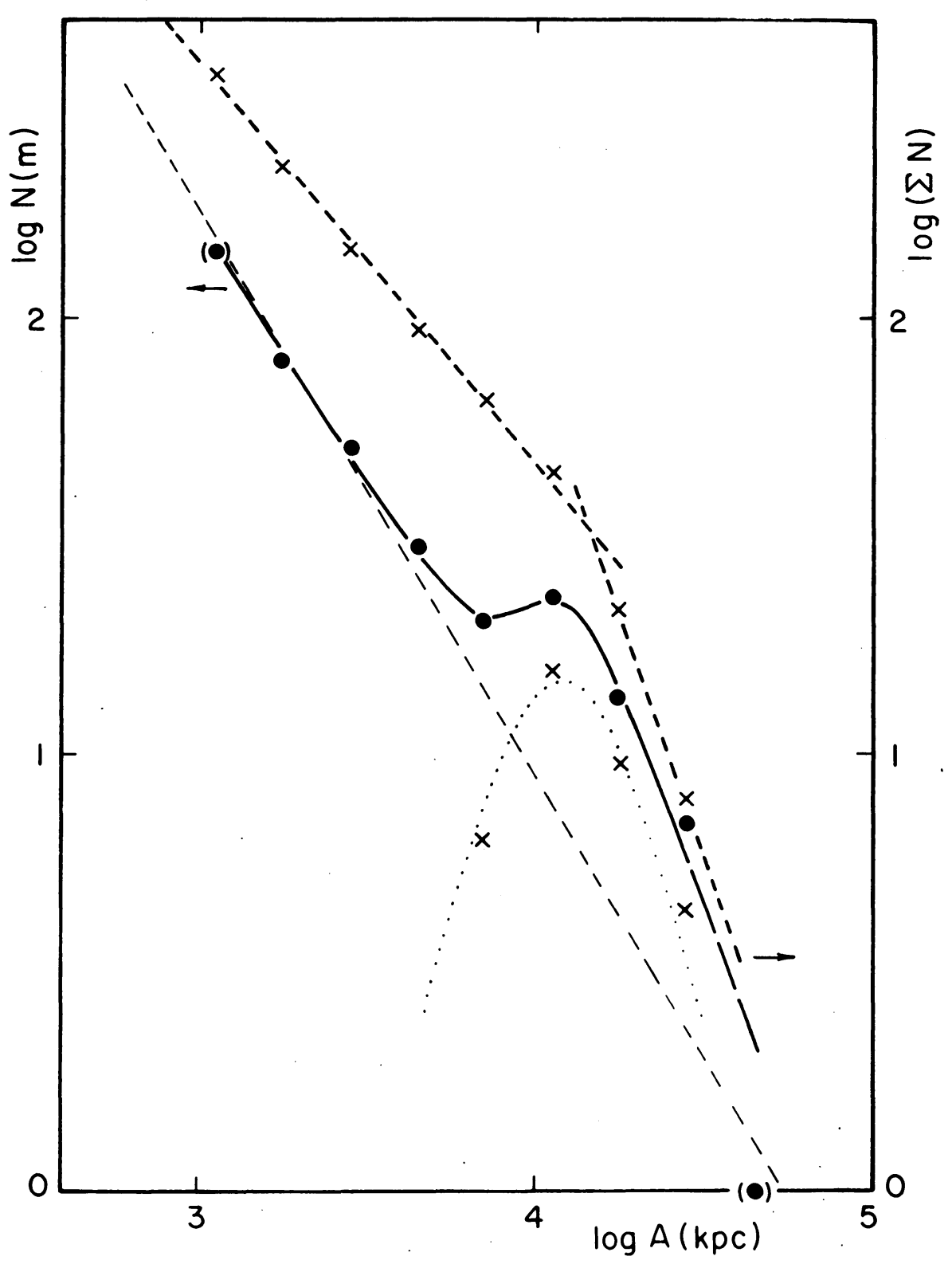

Fig. 26. Frequency function (dots and full line, scale at left, and cumulative frequency function (crosses and dashed lines, scale at right) of linear diameters of galaxies and their companions, after Holmberg (1969). The observed differential function can be resolved into two components, one obeying Hubble's gaussian function (dotted parabola), the other following Zwicky's exponential function (thin dashes). The integrated function can be approximated by two straight segments (heavy dashes) as observed by Abell in clusters (Figure 27). 


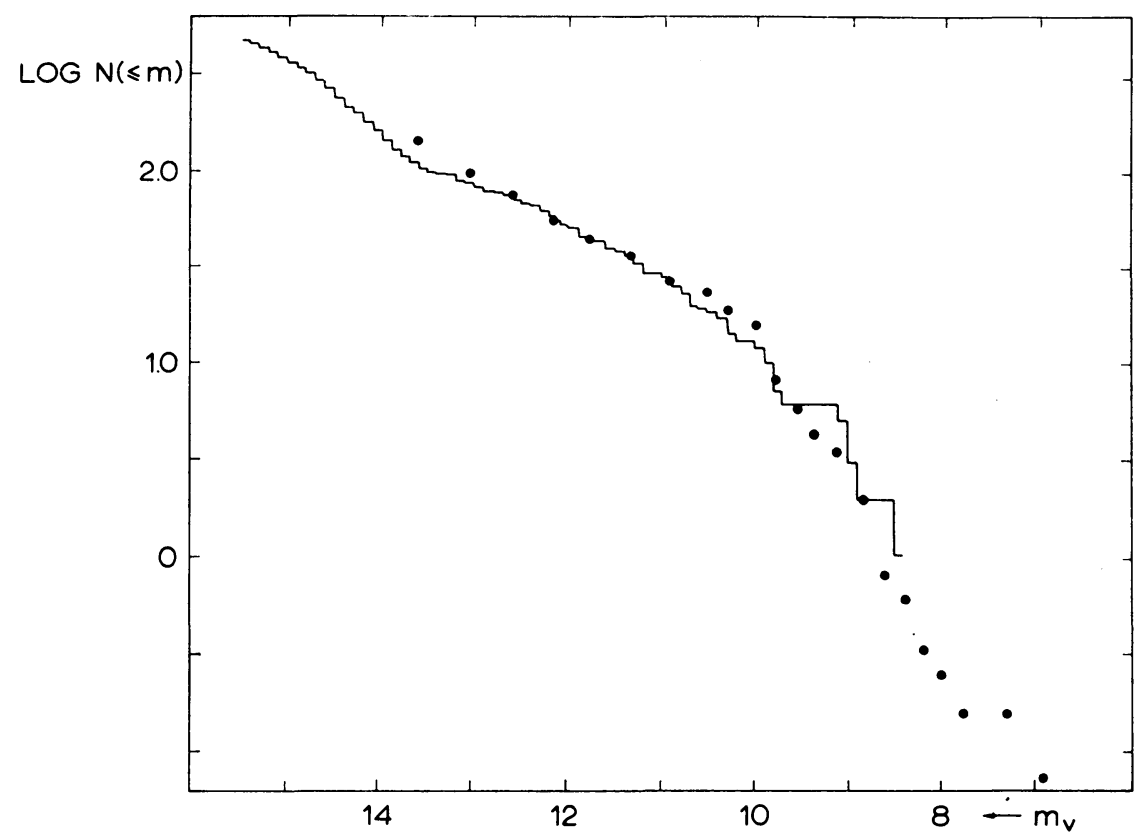

Fig. 27. Integrated luminosity functions of Coma (dots) and Virgo clusters, after Abell (1972).

Scales apply to Virgo cluster.

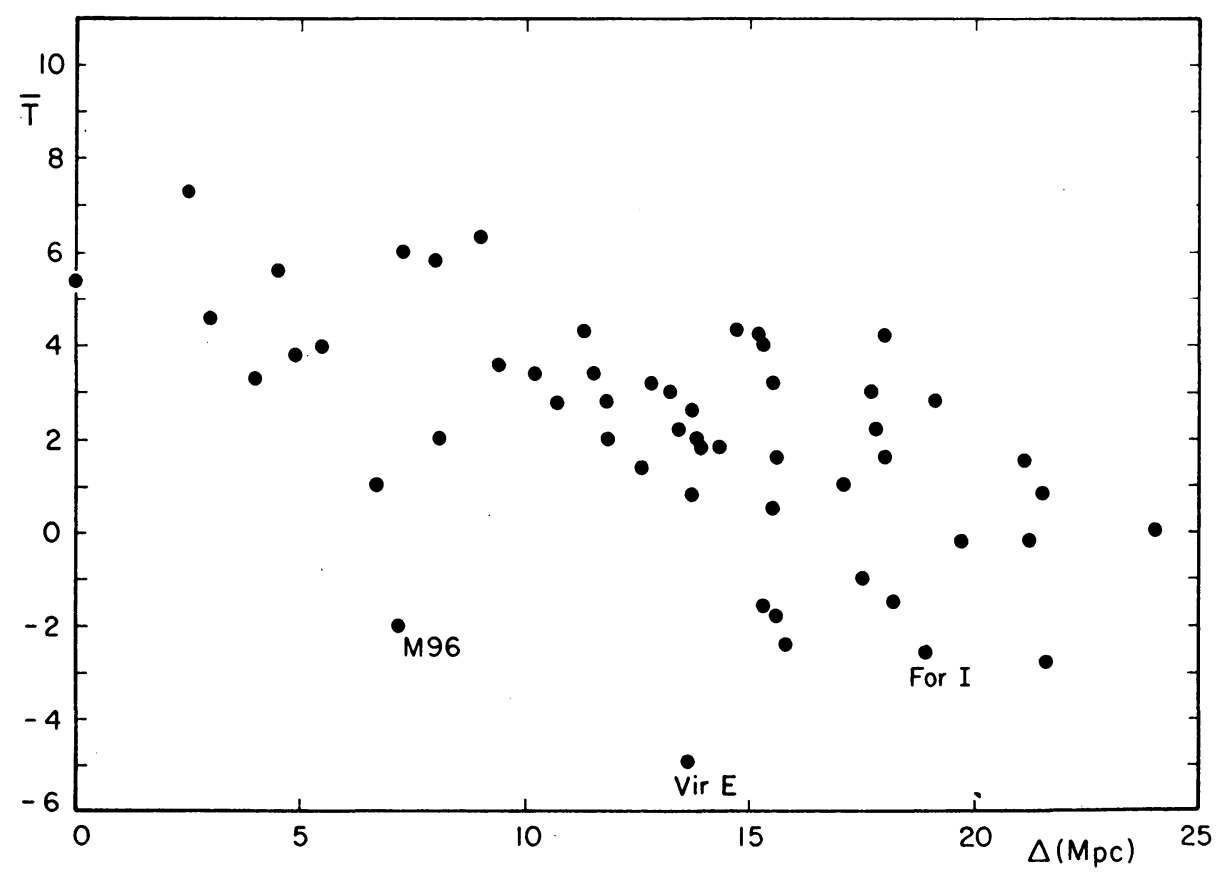

Fig. 28. Average morphological type $t$ of five brightest members of nearby groups as a function of distance $\Delta$ (de Vaucouleurs 1965; revised 1973). The Local Group, at $\Delta=0$, is typical of nearby groups dominated by late-type spirals. 


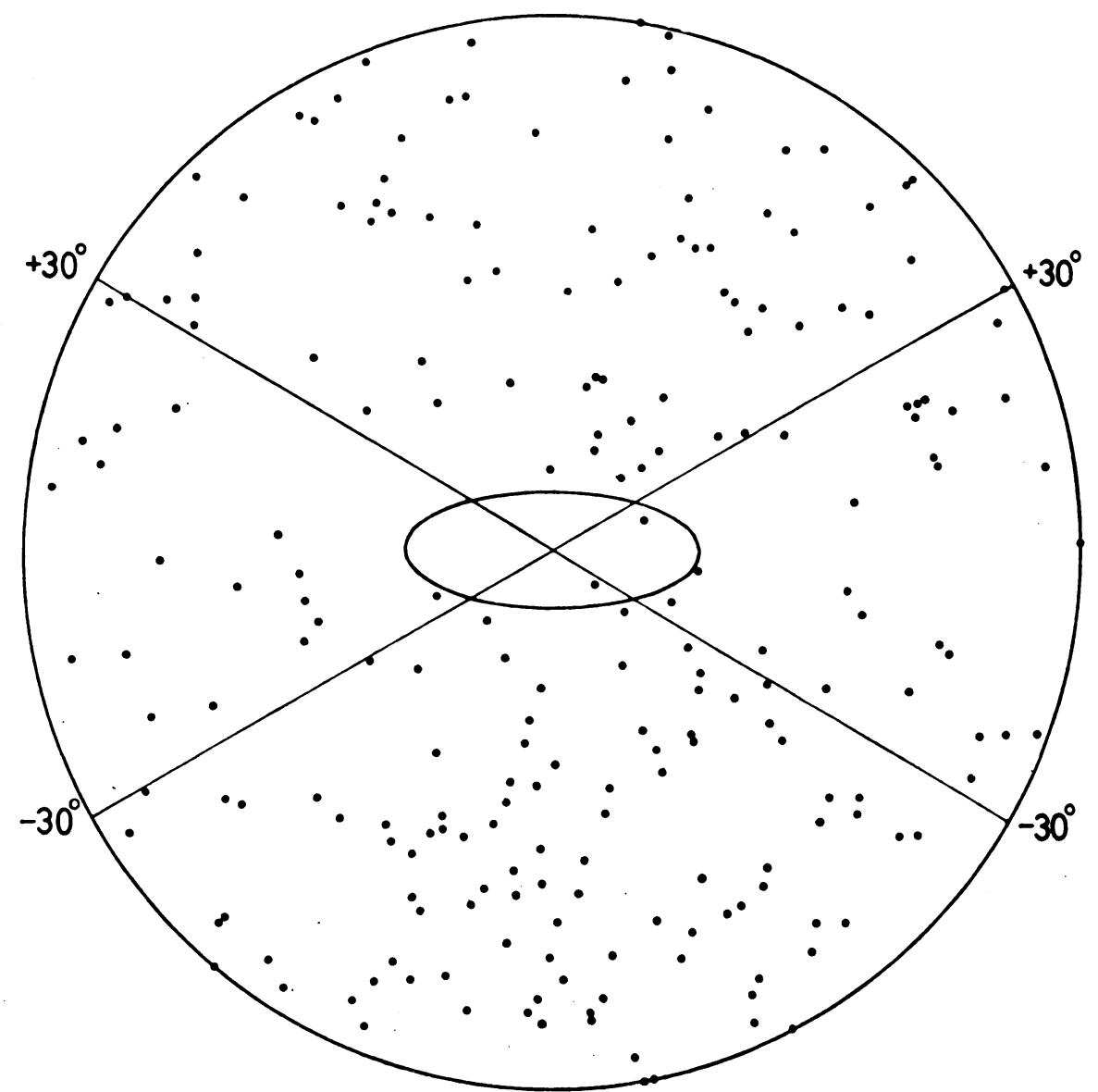

Fig. 29. Distribution of 218 companion galaxies in the vicinity of 58 edge-on systems, after Holmberg (1969). Ellipse shows average size of central system; note excess density within $\pm 60^{\circ}$ from minor axis of primary.

distribution. The 'lines' of galaxies (and quasars) discussed by Arp (1968) are also suggestive of symmetric ejection of condensed matter by giant galaxies, but the physical cause and possible mechanism of the postulated phenomenon elude us.

(4) Giant ellipticals and lenticulars are dominant in dense clusters of galaxies, whether small as the Fornax I cluster or large as Coma I; very few spirals, if any, are members of such clusters. The rare exceptions may be interlopers (captures, encounters or optical coincidences). It is possible that apparently mixed clusters, such as Virgo I, are optical superpositions of a cluster of E, $L$ types and a cloud of $S$ types (de Vaucouleurs, 1961; G. and A. de Vaucouleurs, 1973), but even if the two systems were in fact concentric and co-extensive (Kowal, 1969; Tammann, 1972) the drastically different radial distributions of galaxies of different morphological types $(t<0 \mathrm{vs} t>0)$ remain unexplained. This strong segregation of galaxies of different morphological 
types in groups and clusters of different structural forms has been known and discussed for more than a decade (de Vaucouleurs, 1956a, 1962, 1965; Morgan, 1958b) but it has not yet received the attention it deserves from the point of view of galaxy formation and/or evolution.

\section{Maximum Space Density in Galaxies and Clusters}

Selection effects discriminate mainly against small and faint galaxies; large and/or bright objects dominate our catalogues. Among objects having a given diameter - the effective diameter, say, for definiteness - a range of masses and densities exist. While the lower limits may be set more by selection factors than by physical conditions, the upper limits should not be so biased.

From such data (Table VII) we may, perhaps, answer two important questions:

(1) Is there an upper limit to the mass (or density) present in a given volume of space?

(2) What is the mass-radius relation for the densest objects or regions of space?

It is remarkable that the densest known galaxies, groups, clusters and superclusters

TABLE VII

Mass-radius-density data ${ }^{\mathrm{a}}$

\begin{tabular}{|c|c|c|c|c|}
\hline Class of Objects & Examples & $\begin{array}{l}\log \mathfrak{M}_{R} \\
(\mathrm{~g})\end{array}$ & $\begin{array}{l}\log R^{\mathrm{b}} \\
(\mathrm{cm})\end{array}$ & $\begin{array}{l}\log Q \\
\left(\mathrm{~g} \mathrm{~cm}^{-3}\right)\end{array}$ \\
\hline Centres of dense nuclei & $\begin{array}{l}\text { M31, Centre } \\
(R=1 \mathrm{pc})\end{array}$ & - & 18.5 & $-16.5:$ \\
\hline Dense nuclei & M31, nucleus & 40.3 & 19.3 & -18.2 \\
\hline of galaxies & M32, core & 41.0 & 19.5: & -18.1 \\
\hline Compact dwarf & M32 & 42.5 & 20.65 & -20.0 \\
\hline ellipticals & N4486-B & 43.2 & 21.1 & -20.7 \\
\hline Normal giant & M33 & 43.5 & 21.8 & -22.5 \\
\hline spirals & M31 & 44.6 & 22.3 & -22.9 \\
\hline \multirow[t]{2}{*}{ Giant ellipticals } & N3378 & 44.3 & 22.0 & -22.35 \\
\hline & N4486 & 45.1 & 22.5 & -22.95 \\
\hline $\begin{array}{l}\text { Cor: pact groups } \\
\text { of spin ils }\end{array}$ & $\begin{array}{l}\text { Seyfert, } \\
\text { Stephan }\end{array}$ & 45.5 & 22.6: & -23.1 \\
\hline $\begin{array}{l}\text { Small dense clusters } \\
\text { of ellipticals }\end{array}$ & $\begin{array}{l}\text { Virgo E, core } \\
\text { Fornax I }\end{array}$ & 46.5 & 23.7 & -25.2 \\
\hline $\begin{array}{l}\text { Small loose groups } \\
\text { of spiraıs }\end{array}$ & Nearby groups & 46.5 & 24.1 & -26.4 \\
\hline $\begin{array}{l}\text { Small clouds } \\
\text { of spirals }\end{array}$ & $\begin{array}{l}\text { Virgo S, } \\
\text { Ursa Major }\end{array}$ & 47.0 & 24.3 & -26.5 \\
\hline $\begin{array}{l}\text { Small clusters } \\
\text { of ellipticals }\end{array}$ & Virgo E & 47.2 & 24.3 & -26.3 \\
\hline $\begin{array}{l}\text { Large clusters } \\
\text { of ellipticals }\end{array}$ & Coma I & 47.9 & 24.5 & -26.2 \\
\hline Superclusters & Local & 48.7: & 25.5 & $-28.4:$ \\
\hline
\end{tabular}


of galaxies of increasing radii define a rather tight correlation over the large range of radii $10^{18}<R<10^{26} \mathrm{~cm}$ (de Vaucouleurs, 1970b, 1971) (Figure 30). In this range the maximum space density observed in various volumes centred on galaxies and systems of galaxies is given (in cgs units) by

$$
\log \varrho_{\mathrm{R}}=-21.7-1.7(\log R-21.7) \text {. }
$$

This maximum density is from 4 to 6 orders of magnitude lower than the Schwarzschild

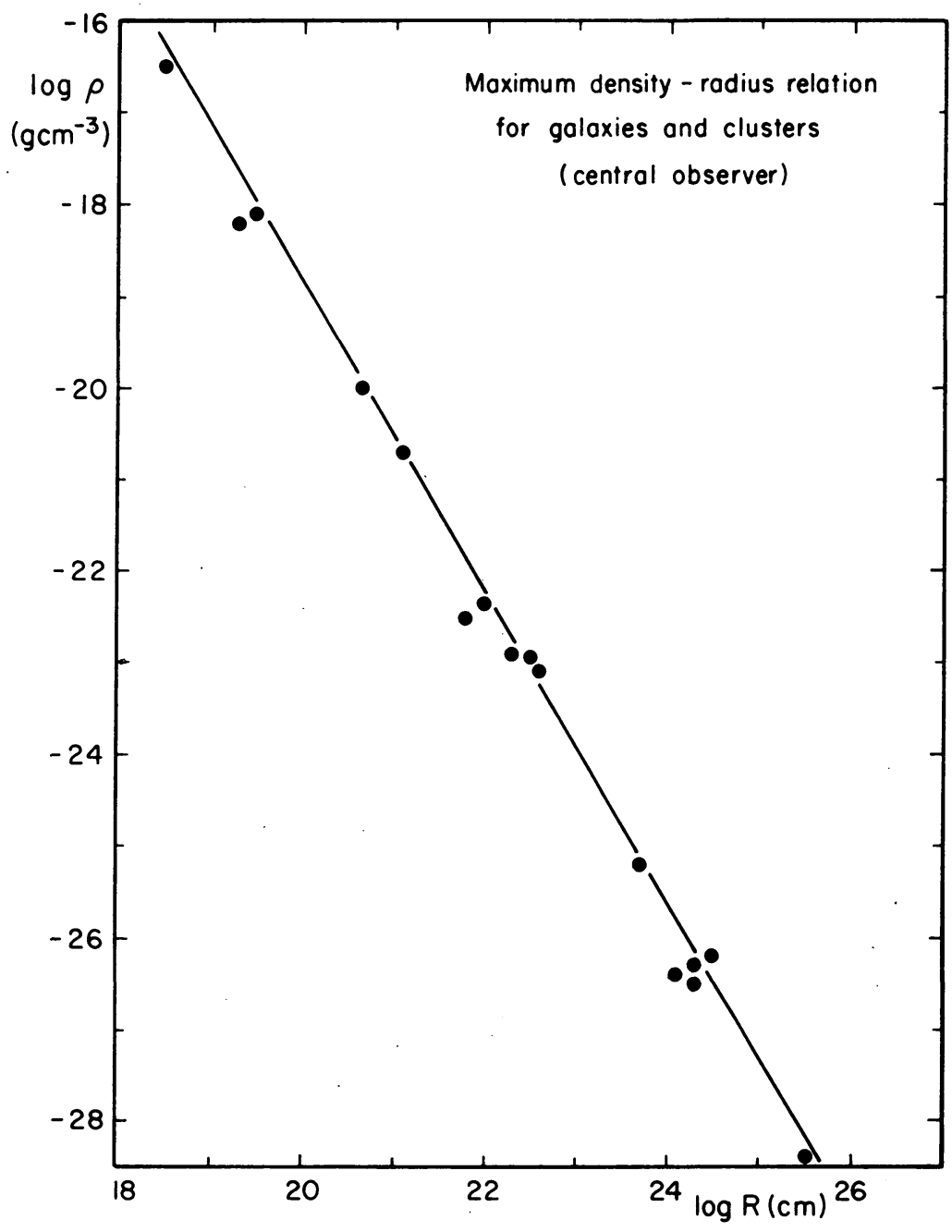

Fig. 30. Correlation between maximum density and radius of spheres centred on galaxies and systems of galaxies (de Vaucouleurs, 1971). The maximum density observable in a given volume of space by a central observer has an upper bound outlined here by some examples in the range $(18<\log R<26)$. 
limit, $\log \varrho_{\mathrm{s}}=27.2-2 \log R_{\mathrm{s}}$. Whether the relation extends to systems larger than superclusters $(\log R>26)$ or not is still in doubt.*

Since $\varrho$ and $R$ are related through the virial theorem, another expression of this result is a relation between velocity dispersion $\sigma_{v}$ and cluster radius for groups and clusters of galaxies; according to Karachentsev (1967) and Ozernoy (1969) the relation

$$
\log \sigma_{\mathrm{v}}=0.40 \log R+\text { constant (cgs) }
$$

applies in the range $23<\log R<26$ for 143 systems of galaxies (pairs to supercluster). Still another expression of this correlation is the much discussed apparent dependence of the 'missing mass' ratio upon the multiplicity of galaxy systems (Page, 1965; Rood et al., 1970). Reference is made to a recent review article on galaxy clustering for further details (de Vaucouleurs, 1971).

\section{References}

Abell, G. O.: 1962, in G. C. McVittie (ed.), 'Problems of Extragalactic Research', IAU Symp. 15, 213. Abell, G. O.: 1972, in D. S. Evans (ed.), 'External Galaxies and Quasi-Stellar Objects', IAU Symp. 44, 341.

Ables, H. D.: 1971, Publ. U.S. Naval Obs. XX, Part IV.

Arp, H. C.: 1965, Astrophys. J. 142, 402.

Arp, H. C.: 1966, Atlas of Peculiar Galaxies, Calif. Inst. of Technology, Pasadena.

Arp, H. C.: 1968, Publ. Astron. Soc. Pacific 80, 129.

Arp, H. C. and Bertola, F.: 1969, Astrophys. Letters 4, 23.

Arp, H. C. and Bertola, F.: 1971, Astrophys. J. 163, 195.

Baade, W. and Minkowski, R.: 1954, Astrophys. J. 119, 225.

Balkowski, C.: 1972, Etude statistique des propriétés intégrales des galaxies mesurées en raie $21 \mathrm{~cm}$, Thesis, Univ. of Paris.

Balkowski, C., Bottinelli, L., Gouguenheim, L., and Heidmann, J.: 1972, Astron. Astrophys. 21, 203. Baum, W.: 1959, Publ. Astron. Soc. Pacific 71, 106.

Bonnor, W. B.: 1972, Monthly Notices Roy. Astran. Soc. 159, 261.

Bottinelli, L.: 1970, La distribution spatiale à grande échelle de l'hydrogéne neutre dans les galaxies, Thesis, Univ. of Paris.

Bottinelli, L.: 1971, Astron. Astrophys. 10, 437.

Bottinelli, L., Gouguenheim, L., Heidmann, J., and Heidmann, N.: 1968, Ann. Astrophys. 31, 205. Bottinelli, L., Gouguenheim, L., and Heidmann, J.: 1973, Astron. Astrophys. 25, 451.

Brandt, J. C.: 1960, Astrophys. J. 131, 293.

Brandt, J. C. and Roosen, R. G.: 1969 Astrophys. J. Letters 156, L59.

Brosche, P.: 1963, Z. Astrophys. 57, 143.

Brosche, P.: 1971, Astron. Astrophys. 13, 293.

Brosche, P.: 1973, Astron. Astrophys. 23, 259.

Burbidge, E. M. and Burbidge, G. R.: 1960, Astrophys. J. 131, 224.

Burbidge, E. M. and Burbidge, G. R.: 1972, Astrophys. J. 171, 253.

Burbidge, E. M., Burbidge, G. R., and Prendergast, K. H.: 1960, Astrophys. J. 132, 654.

Burbidge, E. M., Burbidge, G. R., and Fish, R. A.: 1961, Astrophys. J. 134, 251.

Burbidge, E. M., Burbidge, G. R., and Prendergast, K. H.: 1963, Astrophys. J. 137, 376.

* This density-radius relation has often been grossly misunderstood and incorrectly applied (Haggerty and Wertz, 1972; Sandage et al., 1972; Bonnor, 1972). Equation (14) applies only to centrally located observers in the densest known systems of each size; it must be understood as describing an upper bound to the space densities seen by central observers. It should be obvious that it is not an expression for the average density observed from an arbitrary location in space. 
Code, A. D., Welch, G. A., and Page, T. L.: 1972, in Scientific Results from the Orbiting Astronomical Observatory $(O A O-2)$, NASA SP-310, p. 559.

Corwin, H. G.: 1967, Galaxy Groups, M. A. Thesis, Univ. of Kansas.

Corwin, H. G.: 1968, Publ. Dept. Astron. Univ. of Texas, Austin, II, No. 12.

Corwin, H. G.: 1970, Publ. Dept. Astron. Univ. of Texas, Austin, III, No. 5.

Danby, J. M. A.: 1965, Astron. J. 70, 501.

Deeming, T. J.: 1968, Vistas in Astronomy 10, 125.

de Vaucouleurs, G.: 1948, Compt. Rend. Acad. Sci. Paris 227, 548.

de Vaucouleurs, G.: 1953, Monthly Notices Roy. Astron. Soc. 113, 134.

de Vaucouleurs, G.: 1955, Astron. J. 60, 126, 319.

de Vaucouleurs, G.: 1956a, Mem. Comm. Obs. Mt. Stromlo, III, No. 13.

de Vaucouleurs, G.: 1956b, Irish Astron. J. 4, 13.

de Vaucouleurs, G.: 1957a, Ann. Obs. Le Houga II, fasc. 1.

de Vaucouleurs, G.: 1957b, Astron. J. 62, 69.

de Vaucouleurs, G.: 1958a, Astrophys. J. 128, 465.

de Vaucouleurs, G.: 1958b, Astron. J. 63, 253.

de Vaucouleurs, G.: 1959a, Astron. J. 64, 397.

de Vaucouleurs, G.: 1959b, in Handbuch der Physik 53, Springer-Verlag, Berlin, Göttingen, p. 275, 311.

de Vaucouleurs, G.: 1960, Astrophys. J. 131, 585.

de Vaucouleurs, G.: 1961, Astrophys. J. Suppl. 5, No. 48, 233.

de Vaucouleurs, G.: 1962, in G. C. McVittie (ed.), 'Classification of Galaxies by Form, Luminosity and Color', IAU Symp. 15, 3.

de Vaucouleurs, G.: 1963, Astrophys. J. Suppl. 8, No. 74, 31.

de Vaucouleurs, G.: 1964, Astron. J. 69, 737.

de Vaucouleurs, G.: 1965, 'Nearby Groups of Galaxies', Stars and Stellar Systems, 9, Chapter 17 (in press).

de Vaucouleurs, G.: 1969, Astrophys. Letters 4, 17.

de Vaucouleurs, G.: 1970a, Bull. Am. Astron. Soc. 2, 308.

de Vaucouleurs, G.: 1970b, Science 167, 1203.

de Vaucouleurs, G.: 1971, Publ. Astron. Soc. Pacific 83, 113.

de Vaucouleurs, G.: 1973, Report to Commission 30, IAU meeting, Sydney.

de Vaucouleurs, G. and Ables, H.: 1968, Astrophys. J. 151, 105.

de Vaucouleurs, G. and Agüero, E.: 1973, Publ. Astron. Soc. Pacific 85, 150.

de Vaucouleurs, G. and Freeman, K. C.: 1972, Vistas in Astronomy 14, 163.

de Vaucouleurs, G. and de Vaucouleurs, A.: 1959a, Lowell Obs. Bull. IV, No. 92, 58.

de Vaucouleurs, G. and de Vaucouleurs, A.: 1959b, Publ. Astron. Soc. Pacific 71, 83.

de Vaucouleurs, G. and de Vaucouleurs, A.: 1964, Reference Catalogue of Bright Galaxies, The Univ. of Texas Press, Austin.

de Vaucouleurs, G. and de Vaucouleurs, A.: 1970, Astrophys. Letters 5, 219.

de Vaucouleurs, G. and de Vaucouleurs, A.: 1972, Mem. Roy. Astron. Soc. 77, Part I, 1.

de Vaucouleurs, G. and de Vaucouleurs, A.: 1973, Astron. Astrophys. 28, 109.

de Vaucouleurs, G., de Vaucouleurs, A., and De Cesare, M.: 1969a, Bull. Am. Astron. Soc. 1, 186. de Vaucouleurs, G., de Vaucouleurs, A., and De Cesare, M.: 1969b, Contr. McDonald Obs. II, No. 26 (see also Sky Telesc. 37, No. 3)

Einasto, J.: 1968, Publ. Tartu Astron. Obs. 36, 396, 414.

Einasto, J.: 1969, Astrofizika 5, 137.

Einasto, J.: 1970, Astrofizika 6, 149, 241.

Einasto, J.: 1972a, Tartu Astron. Obs., Preprint No. 40.

Einasto, J.: 1972b, in D. S. Evans (ed.), 'External Galaxies and Quasi-Stellar Objects', IAU Symp. 44, 37.

Einasto, J. and Kaasik, A.: 1973, Private communication for presentation to this Symposium.

Faber, S. M.: 1971, Unpublished Ph.D. Thesis, Harvard Univ.

Faber, S. M.: 1973, Astrophys. J. 179, 423.

Fish, R. A.: 1964, Astrophys. J. 139, 284.

Fraser, C. W.: 1972, Observatory $92,51$.

Freeman, K. C.: 1970, Astrophys. J. 160, 811. 
Freeman, K. C. and de Vaucouleurs, G., : 1974, in preparation.

Gallagher III, J. S.: 1972, Astron. J. 77, 568.

Gilra, D. P.: 1971, Nature 229, 237.

Gilra, D. P.: 1972, in Scientific Results from the Orbiting Astronomical Observatory (OAO-2), NASA SP-310, p. 295.

Gordon, K. J.: 1971, Astrophys. J. 169, 235.

Gouguenheim, L.: 1969, Astron. Astrophys. 3, 281.

Gursky, H., Kellogg, E., Murray, S., Leong, C., Tananbaum, H., and Giacconi, R.: 1971, Astrophys. J. Letters 165, L49.

Haggerty, M. J. and Wertz, J. R.: 1972, Monthly Notices Roy. Astron. Soc. 155, 495.

Heidmann, J.: 1967, Compt. Rend. Acad. Sci. Paris 263B, 1186.

Heidmann, J.: 1969, Astrophys. Letters 3, 19.

Heidmann, J., Heidmann, N., and de Vaucouleurs, G.: 1971, Mem. Roy. Astron. Soc. 75, Parts 4-6, $85,105,121$.

Heidmann, N.: 1969, Astrophys. Letters 3, 153, and Thesis, Univ. of Paris.

Herschel, J.: 1847, in Results of Astronomical Observations Made During the Years 1834, 5, 6, 7, 8 at the Cape of Good Hope, Smith \& Co., London.

Hodge, P. W.: 1961, Astron. J. 66, 249, 384.

Hodge, P. W.: 1963, Astron. J. 68, 470.

Hodge, P. W.: 1964, Astron. J. 69, 438.

Hodge, P. W.: 1965, Astrophys. J. 142, 1390.

Hodge, P. W.: 1973, Astrophys. J. 182, 671.

Hodge, P. W. and Hitchcock, J. L.: 1966, Publ. Astron. Soc. Pacific 78, 79.

Hodge, P. W. and Michie, R. W.: 1969, Astron. J. 74, 587.

Holmberg, E.: 1946, Medd. Lund Obs. Ser II, No. 117.

Holmberg, E.: 1950, Medd. Lund Obs. Ser. II, No. 128.

Holmberg, E.: 1958, Medd. Lund Obs. Ser. II, No. 136.

Holmberg, E.: 1964, Arkiv Astron. 3, 387 (=Medd. Uppsala Obs. No. 148).

Holmberg, E.: 1969, Arkiv Astron. 5, 305 (=Medd. Uppsala Obs. No. 166).

Hubble, E.: 1926, Astrophys. J. 64, 321.

Hubble, E.: 1930, Astrophys. J. 71, 231.

Hubble, E.: 1936, Astrophys. J. 84, 158.

Huchtmeier, W.: 1973, Astron. Astrophys. 22, 91.

Humason, M. L., Mayall, N. U., and Sandage, A. R.: 1956, Astron. J. 61, 97.

Johnson, H. L.: 1966, Astrophys. J. 143, 187.

Johnson, H. M.: 1961, Astrophys. J. 133, 314.

Karachentsev, I. D.: 1967, Commun. Byurakan Obs. 39, 96.

Karachentseva, V. E.: 1967, Astrofizika 3, 535.

Karachentseva, V. E.: 1972, in Problems of Cosmical Physics, Kiev Univ. Publ. No. 7, p. 150.

Kellogg, E., Gursky, H., Leong, C., Schreier, E., Tananbaum, H., and Giacconi, R.: 1971, Astrophys. J. Letters 165, L49.

Kerr, F. J.: 1973, Report to Commission 40, IAU meeting, Sydney.

Kiang, T.: 1961, Monthly Notices Roy. Astron. Soc. 122, 263.

King, I. R.: 1961, Astron. J. 66, 68.

King, I. R.: 1962, Astron. J. 67, 471.

King, I. R.: 1966, Astron. J. 71, 64, 276.

King, I. R. and Kiser, J.: 1973, Astrophys. J. 187, 27.

King, I. R. and Minkowski, R.: 1972, in D. S. Evans (ed.), 'External Galaxies and Quasi-Stellar Objects', IAU Symp. 44, 87.

Kinman, T. D.: 1965, Astrophys. J. 142, 1376.

Kleinmann, D. E. and Low, F. J.: 1970a, Astrophys. J. Letters 159, L165.

Kleinmann, D. E. and Low, F. J.: 1970b, Astrophys. J. Letters 161, L203.

Kogure, T. and Toya, N.: 1970, Mem. Fac. Sci. Kyoto Univ. XXXIII, No. 2 (Dept. Astron. Kyoto Univ. Rep. No. 41).

Kowal, C. T.: 1969, Publ. Astron. Soc. Pacific 81, 608.

Kuzmin, G. G.: 1952, Publ. Astron. Obs. Tartu 32, 211.

Lasker, B. M.: 1970, Astron. J. 75, 21. 
Martin, W. L. and Bingham, R. G.: 1970, Observatory 90, 13.

Mathews, T. A., Morgan, W. W., and Schmidt, M.: 1964, Astrophys. J. 140, 35.

Mayall, N. U.: 1939, Lick Obs. Bull. 19, No. 497, 33.

Mayall, N. U.: 1948, Sky Telesc. 8, 3.

Mayall, N. U.: 1960, Ann. Astrophys. 23, 344 (=Lick Obs. Bull. No. 566).

Mayall, N. U. and Lindblad, P. O.: 1970, Astron. Astrophys. 8, 364.

McClure, R. D.: 1969, Astron. J. 74, 50.

McClure, R. D. and van den Bergh, S.: 1968, Astron. J. 73, 313, 1008.

McGee, R. X.: 1964, Australian J. Phys. 17, 515.

McGee, R. X. and Milton, J. A.: 1966a, Australian J. Phys. 19, 433.

McGee, R. X. and Milton, J. A.: 1966b, Australian J. Phys., Astrophys. Suppl. No. 2.

Mestel, L.: 1963, Monthly Notices Roy. Astron. Soc. 126, 553.

Minkowski, R.: 1962, in G. C. McVittie (ed.), 'Problems of Extragalactic Research', IAU Symp. $15,112$.

Minkowski, R. and Osterbrock, D.: 1959, Astrophys. J. 129, 583.

Mitchie, R. W.: 1963, Monthly Notices Roy. Astron. Soc. 125, 127.

Morgan, W. W.: 1958a, Publ. Astron. Soc. Pacific 70, 372.

Morgan, W. W.: 1958b, in R. Stoops (ed.), La Structure et l'Evolution de l'Univers, Solvay Conference

Rep., Bruxelles, p. 297.

Morton, D. C. and Chevalier, R. A.: 1972, Astrophys. J. 174, 489.

Morton, D. C. and Chevalier, R. A.: 1973, Astrophys. J. 179, 55.

Oemler, A.: 1973, Astrophys. J. 180, 11.

Oort, J.: 1940, Astrophys. J. 91, 273.

Oort, J.: 1965, Trans. IAU XIIA, 789.

Osterbrock, D.: 1960, Astrophys. J. 132, 325.

Owen, F. N.: 1973, Radio Sources in Clusters of Galaxies, Ph.D. Dissert., Univ. of Texas at Austin. Ozernoy, L. M.: 1967, Astron. Tsirk. USSR, No. 407.

Ozernoy, L. M.: 1969, Zh. Eksperim. Teor. Fiz. (Letters) 10, 394 (=JETP Letters 10, 251)

Page, T. L.: 1965, Smithsonian Astrophys. Obs. Special Rep. No. 195.

Perek, L.: 1950, Bull. Astron. Inst. Czech. 2, 75.

Poveda, A.: 1958, Bol. Obs. Tonantzintla Tacubaya No. 17.

Poveda, A.: 1961, Astrophys. J. 134, 910.

Poveda, A., Iturriaga, R., and Orozco, I.: 1960, Bol. Obs. Tonantzintla Tacubaya No. 20, 3.

Randers, G.: 1940, Astrophys. J. 92, 235.

Reeves, G.: 1956, Astron. J. $61,69$.

Rieke, G. H. and Low, F. J.: 1972, Astrophys. J. Letters 176, L95.

Roberts, M. S.: 1966, Astrophys. J. 144, 639.

Roberts, M. S.: 1969, Astron. J. 74, 859.

Roberts, M. S.: 1972, in D. S. Evans (ed.), 'External Galaxies and Quasi-Stellar Objects', IAU Symp. 44, 12.

Rood, H. J.: 1965, Astron. J. 70, 689.

Rood, H. J., Rothman, V. C. A., and Turnrose, B. E.: 1970, Astrophys. J. 162, 411.

Rubin, V. C., Burbidge, E. M., Burbidge, G. R., and Prendergast, K. H.: 1964, Astrophys. J. 140, 80.

Sandage, A.: 1961, The Hubble Atlas of Galaxies, Publ. 618, Carnegie Inst. of Washington, D. C.

Sandage, A.: 1963, Astrophys. J. 138, 863.

Sandage, A.: 1972, Astrophys. J. 176, 21.

Sandage, A.: 1973, Stars and Stellar Systems 9, in press.

Sandage, A., Freeman, K. C., and Stokes, N. R.: 1970, Astrophys. J. 160, 831.

Sandage, A., Tammann, G. A., and Hardy, E.: 1972, Astrophys. J. 172, 253.

Sargent, W. L. W.: 1970, Astrophys. J. 160, 405.

Sargent, W. L. W. and Searle, L.: 1970, Astrophys. J. Letters 162, L155.

Saslaw, W. C.: 1970, Astrophys. J. 160, 11.

Saslaw, W. C.: 1971a, Astrophys. J. 163, 249.

Saslaw, W. C.: 1971b, Monthly Notices Roy. Astron. Soc. 152, 351.

Saslaw, W. C.: 1972, in D. S. Evans (ed.), 'External Galaxies and Quasi-Stellar Objects', IAU Symp. 44, 93.

Schild, R. and Oke, J. B.: 1971, Astrophys. J. 169, 209. 
Schilt, J., Epstein, I., and Hill, S. J.: 1955, Astron. J. 60, 341.

Sersic, J. L.: 1957, Revista Astron. XXIX-II, 68 (see also Observatory 78, 24, 1958).

Sersic, J. L.: 1966, Z. Astrophys. 64, 202.

Sersic, J. L.: 1968a, in 'Non-Stable Phenomena in Galaxies', IAU Symp. 29, Acad. Sci. Armen. SSR, Yerevan, p. 403.

Sersic, J. L.: 1968b, Atlas de Galaxias Australes, Obs. Astron., Cordoba, Argentina.

Smith, R. T.: 1941, Publ. Astron. Soc. Pacific 53, 187.

Spinrad, H.: 1966, Publ. Astron. Soc. Pacific 78, 370.

Spinrad, H., Gunn, J. E., Taylor, B. J., McClure, R. D., and Young, J. W.: 1971, Astrophys. J. 164, 11.

Takase, B.: 1967, Publ. Astron. Soc. Japan 19, 427.

Takase, B. and Kinoshita, H.: 1967, Publ. Astron. Soc. Japan 19, 209.

Tammann, G. A.: 1972, Astron. Astrophys. 21, 355.

Tifft, W. G.: 1961, Astron. J. 66, 390.

Tifft, W. G.: 1963, Astron. J. 68, 302.

Tifft, W. G.: 1969, Astron. J. 74, 354.

Toomre, A. and Toomre, J.: 1972, Astrophys. J. 178, 623.

van den Bergh, S.: 1959, Publ. David Dunlap Obs. II, No. 5, 147.

van den Bergh, S.: 1960, Astrophys. J. 131, 558.

van den Bergh, S.: 1961, Z. Astrophys. 53, 219.

van den Bergh, S.: 1968, J. Roy. Astron. Soc. Canada 62, No. 2, 3; Commun. David Dunlap Obs.

No. 195.

van den Bergh, S.: 1972a, in D. S. Evans (ed.), 'External Galaxies and Quasi-Stellar Objects', IAU Symp. 44, 1.

van den Bergh, S.: 1972b, J. Roy. Astron. Soc. Canada 66, 237.

van Houten, C. J.: 1961, Bull. Astron. Inst. Neth. 16, 1.

von Hoerner, S.: 1957, Astrophys. J. 125, 451.

Vorontsov-Velyaminov, B. A.: 1959, Atlas and Catalogue of Interacting Galaxies, Sternberg Astron. Inst., Moscow.

Vorontsov-Velyaminov, B. A.: 1962, Morphological Catalogue of Galaxies I, Moscow.

Vorontsov-Velyaminov, B. A.: 1967, in Modern Astrophysics, Gauthier-Villars, Paris, p. 347.

Vorontsov-Velyaminov, B. A.: 1970a, Comm. Sternberg Astron. Inst. Moscow No. 166, p. 3.

Vorontsov-Velyaminov, B. A.: 1970b, Astron. Zh. 47, 271 (=Soviet Astron. 14, 222).

Vorontsov-Velyaminov, B. A.: 1970c, Astron. Zh. 47, 16 (=-Soviet Astror. 14, 11).

Vorontsov-Velyaminov, B. A. and Noskova, R. I.: 1971, Astron. Zh. 48, 513 (=Soviet Astron. 15, 402).

Walker, M. F., Blanco, V. M., and Kunkel, W. E.: 1969, Astron. J. 74, 44, 123.

Warner, P. J., Wright, M. C. H., and Baldwin, J. E.: 1973, Monthly Notices Roy. Astron. Soc. 163, 163.

Wells, D. C.: 1972, Integrated Spectral Energy Distributions of Galaxies, Ph.D. Dissert. Univ. of Texas, Austin.

Wells, D. C.: 1973, Bull. Am. Astron. Soc. 5, 26.

Welsh, G. A. and Sastry, G. N.: 1971, Astrophys. J. Letters 169, L3.

Westerlund, B. E. and Wall, J. V.: 1969, Astron. J. 74, 335.

Whitford, A. E.: 1971, Astrophys. J. 169, 215.

Wood, D. B.: 1966, Astrophys. J. 145, 36.

Woolley, R. v. d. R.: 1954, Monthly Notices Roy. Astron. Soc. 114, 191.

Woolley, R. v. d. R. and Robertson, D. A.: 1956, Monthly Notices Roy. Astron. Soc. 116, 288.

Zonn, W.: 1968, Acta Astron. 18, 338.

Zwicky, F.: 1937, Astrophys. J. 86, 217.

Zwicky, F.: 1957, Morphological Astronomy, Springer-Verlag, Berlin.

\section{DISCUSSION}

Schild: Your slides of the concentration index and other luminosity distribution-related parameters vs Hubble type showed poor correlation, and you regretted being unable to derive quantitative Hubble types. Is not the integrated spectral type, or what may be more quantitatively measured, the integrated 
energy distribution, a very much better indicator of Hubble type, especially if the absolute magnitudes are approximately known from the magnitude and redshift?

G. de Vaucouleurs: No, colour indices depend on too many extraneous variables (inclination, redshift, galactic extinction, line emission, etc.), and are not usually measured precisely enough to yield a good equivalent Hubble type (see e.g. G. \& A. de Vaucouleurs: 1972, Mem. Roy. Astron. Soc. 77, Part 1). Neutral hydrogen mass, or density, or $M_{\mathrm{H}} / M_{\mathrm{T}}$, etc. likewise give only poor correlations at present. From principal component analysis of $T$ val iables, Brosche (Astron. Astrophys. 23, 259, 1973) has demonstrated that the standard error of the Reference Catalogue revised Hubble types is $\sigma_{\mathrm{T}}=0.75$ or only $5 \%$ of the range of the $T$ variable.

van den Bergh: If E0 galaxies are indeed flattened objects seen pole-on, then how is one to interpret the geometry of an E0 galaxy like M87 in which the inner isophotes are circular and the outer isophotes are elongated?

G. de Vaucouleurs: I have no good answer to this good question. Note, however, that the statistics of ellipticities refer to $\mu_{\mathrm{B}}=25 \mathrm{mag}$ ( $\left.\operatorname{arc~sec}\right)^{-2}$ isophotes, not to the very low luminosity outer coronas which may be subject to tidal interactions (or mixing) in dense regions such as the core of the Virgo cluster. Also it may no longer be justifiable to classify M87 as E0; it may be just the globular nucleus of a larger lenticular object, i.e. a cD galaxy, with a faint flat component (cf. Astrophys. Letters 4, 17, 1969).

Arp: When you classified the compacts off the end of the ellipticals in the Hubble sequence, did you distinguish between red and blue compacts?

G. de Vaucouleurs: The red compacts such as M32, NGC 4486B are at $T=-6$. The emission line - $\mathrm{H}$ II regions - compacts were placed beyond the Im type at $T=+11$. I have no quantitative information on early-type or continuum-blue compacts such as NGC 1510, which may also be placed at $T=-6$. The Hubble sequence is basically one of morphology, not colour.

Arp: That is what I understood, but I have taken spectra of Zwicky-type blue compacts which showed no emission lines whatsoever - only early-type absorption lines. 